\title{
ANALYSIS OF THE ASYMMETRICAL SHORTEST TWO-SERVER QUEUEING MODEL ${ }^{1}$
}

\author{
J.W. COHEN \\ $C W I$ \\ P.O. Box 94079 \\ 1090 GB Amsterdam, The Netherlands
}

(Received September, 1997; Revised February, 1998)

This study presents the analytic solution for an asymmetrical two-server queueing model for arriving customers joining the shorter queue for the case of Poisson arrivals and negative exponentially distributed service times. The bivariate generating function of the stationary joint distribution of the queue lengths is explicitly determined.

The determination of this bivariate generating function requires a construction of four generating functions. It is shown that each of these functions is the sum of a polynomial and a meromorphic function. The poles and residues at the poles of the meromorphic functions can be simply calculated recursively; the coefficients of the polynomials are easily found, in particular, if the asymmetry in the model parameters is not excessively large. The starting point for the asymptotic analysis for the queue lengths is obtained. The approach developed in the present study is applicable to a larger class of random walks modeling asymmetrical two-dimensional queueing processes.

Key words: Asymmetrical Shortest Queue, Analytic Solution, Meromorphic Functions, Queue Length, Distribution, Generating Functions.

AMS subject classifications: $60 \mathrm{~J} 15,60 \mathrm{~K} 25$.

\section{Introduction}

The "two-server shortest queueing" model, also known as the "two queues in parallel" model, has obtained quite some attention in Queueing Theory literature, the greater part of it concerning the symmetrical model. For a short overview of the studies on the symmetrical model, see [12]. The asymmetrical model presents an analytic problem which appeared inaccessible for quite a long time. In the present study, the solution of this problem will be given.

\footnotetext{
${ }^{1}$ This work was supported in part by the European Grant BRA-QMIPS of CEC DG XIII.
} 
The model concerns a two-server system with a Poisson arrival stream of customers with rate $\lambda$. An arriving customer joins the shorter queue if the queues are unequal; if they are equal, he joins the queue in front of server $i$ with probability $\pi_{i}$, $i=1,2$. The server provides customers exponentially distributed service times with service rate $1 / \beta_{i}, i=1,2$. The symmetrical model refers to the case when $\pi_{1}=$ $\pi_{2}=1 / 2, \beta_{1}=\beta_{2}$. The analysis of the queueing model requires the investigation of the stochastic process $\left\{\mathbf{x}_{1}(t), \mathbf{x}_{2}(t), t \geq 0\right\}$ with $\mathbf{x}_{i}(t)$ being the number of customers present with server $i$ at time $t, i=1,2$. The analysis of this stochastic process can be reduced to that of a random walk with state space being the set of lattice points in $\mathbb{R}_{2}$ with integer-valued, nonnegative coordinates.

In applications of the two-server, shortest queueing model, the stationary joint distribution of the queue lengths or its bivariate generating function usually contains all the information required to calculate the various performance characteristics of the model. Approximate techniques have been employed to obtain this information. Usually, they yield the replacement of the infinite state space of the random walk by a finite one. For the resulting process, the Kolmogorov equations for the stationary probabilities are then solved numerically.

Blanc $[4,5]$, applies the power series algorithm. Here it is assumed that the stationary probabilities can be expressed as a power series made of powers of some suitable chosen function of the traffic load to be handled by the servers. Substitution of these series into the Kolmogorov equations then leads to a set of equations, from which the coefficients of these series can be recursively calculated. The results obtained by this approach are quite satisfactory when compared to those obtained by simulation. Actually, this approach is also based on a special truncation of the state space. Unfortunately, a sufficient mathematical justification of this approach is still not available. Adan et al. [2], and Adan [3] present an iterative approach.

Random walks on the lattice in $\mathbb{R}_{2}$ with integer valued, nonnegative coordinates are instrumental in the analytical investigation of a large class of queueing models to date. For such random walks and their application to Queueing Theory, quite some information is presently available concerning a fairly general approach of their analysis, cf. [7, 8]. For the subclass of semi-homogeneous, nearest neighbor random walks, a more effective analytical approach came recently available, cf. $[10,14]$, and in particular for nearest-neighbor random walks with no one-step transition probabilities to the north, the north-east and the east, cf. [9, 11-13]. For this latter case it appeared that the bivariate generating function of the stationary joint distribution of the random walk, if it exists, can be explicitly expressed in terms of meromorphic functions. A meromorphic function is a function which is regular in the whole complex plane, except for at most a finite number of poles in any finite domain.

The random walk to be used in the analysis of the asymmetrical shortest queue is indeed a nearest-neighbor random walk. However, it is not semi-homogeneous, because the one-step transition probabilities at points above the diagonal of the first quadrant differ from those below this diagonal. In fact, this random walk consists of two semi-homogeneous random walks, one at the points above, the other at the points below the diagonal, and they are coupled at the points of the diagonal. These two random walks do not have one-step transition probabilities to the north, the north-east and the east and so, it was conjectured that the bivariate generating function of its stationary distribution can be indeed described in terms of meromorphic functions. This conjecture was the starting point of our analysis and it appeared to be true. 
We continue this introduction with an overview of the sections of the present study.

In Section 2 we establish functional equations for the bivariate generating function of the stationary joint distribution of the queue length process, cf. (2.4). From these equations, the following two equations are derived, cf. (2.9):

i. $\quad \Omega_{2}(\rho)+\frac{a_{1} \tau}{\rho-\tau} B(\tau)+k_{1}(\rho, \tau) \Phi_{0}(\tau)=0$

for a zero-tuple $(\rho, \tau)$ of $h_{1}(\rho, \tau),|\rho| \leq 1,|\tau| \leq 1$;

ii. $\quad \Omega_{1}(r)-\frac{a_{2} t}{r-t} B(t)+k_{2}(r, t) \Phi_{0}(t)=0$,

for a zero-tuple $(r, t)$ of $h_{2}(r, t),|r| \leq 1,|t| \leq 1$.

Here $k_{i}(\cdot, \cdot)$ is a first degree polynomial, and $h_{i}(\cdot, \cdot)$ a second degree polynomial (they are given); the functions, $B(\cdot), \Phi_{0}(\cdot), \Omega_{1}(\cdot)$ and $\Omega_{2}(\cdot)$ are all generating functions which are regular inside the unit disk and continuous on the closure of the unit disk. Next to these conditions, there is the norming condition, which is equivalent to

$$
\frac{1}{a_{1}} \Omega_{2}(1)+\frac{1}{a_{2}} \Omega_{1}(1)=\frac{1}{a_{1}}+\frac{1}{a_{2}}-1, a_{1}=\lambda \beta_{1}, a_{2}=\lambda \beta_{2} .
$$

From the conditions mentioned so far, the functions $B(\cdot), \Phi_{0}(\cdot), \Omega_{i}(\cdot), i=1,2$, have to be determined, cf. (2.10).

In Section 3, properties of the zeros of $h_{1}(\rho, \tau)=0$ and of $h_{2}(r, t)=0$ are described. These zeros are denoted by , cf. (3.9),

$$
\begin{gathered}
\rho^{ \pm}(\tau) \text { or } \tau^{ \pm}(\rho) \text { for } h_{1}(\rho, \tau)=0, \\
r^{ \pm}(t) \text { or } t^{ \pm}(r) \text { for } h_{2}(r, t)=0 .
\end{gathered}
$$

The curve $h_{1}(\rho, \tau)=0$, when traced for real $\rho$ and $\tau$, is a hyperbola, which has one of its branches into the first quadrant. A graph with successive vertical and horizontal edges is inscribed into the branch in the first quadrant; see Figure 3.2. The corner points of such a graph correspond to zeros of $h_{1}(\rho, \tau)$. Analogously, such a graph is inscribed in the branch of $h_{2}(r, t)=0$. Any point of $h_{1}(\rho, \tau)=0$ induces also such a graph on $h_{2}(r, t)=0$ and vice versa. In Figure 3.4, a binary tree is traced, starting at $\tau_{0}^{(0)}$. Out from $\tau_{0}^{(0)}$ a graph, as mentioned above, is constructed on $h_{1}(\rho, \tau)=0$ and on $h_{2}(r, t)=0$. Each corner point of such a graph induces on the other hyperbola again such a graph; only ascending graphs are used here. The set of corner points so obtained constitutes the tree generated by $\tau_{0}^{(0)}$; see for details Section 3. Such a tree, with a properly chosen top $\tau_{0}^{(0)}$, is instrumental in the construction of the functions $B(\cdot), \Phi_{0}(\cdot), \Omega_{i}(\cdot), i=1,2$.

Section 4 starts with the formulation of a lemma. It states that the functions $B(s), \Phi_{0}(s)$ and $\Omega_{i}(s), i=1,2$, can all be continued meromorphically into $|s|>1$. For those meromorphic continuations a set of relations is derived, cf. (4.1-4.4). We mention here two of those relations, viz.

$$
\begin{gathered}
\Omega_{2}\left(\rho^{-}(\tau)\right)+\frac{a_{1} \tau}{\rho^{-}(\tau)-\tau} B(\tau)+k_{1}\left(\rho^{-}(\tau), \tau\right) \Phi_{0}(\tau)=0 \text { for } h_{1}\left(\rho^{-}(\tau), \tau\right)=0 \\
\Omega_{1}\left(r^{-}(\tau)\right)-\frac{a_{2} t}{r^{-}(t)-t} B(t)+k_{2}\left(r^{-}(t), t\right) \Phi_{0}(t)=0 \text { for } h_{2}\left(r^{-}(\tau), \tau\right)=0 .
\end{gathered}
$$


It is assumed, cf. assumption 4.2 , that all poles of $B(s), \Phi_{0}(s), \Omega_{i}(s), i=1,2$, in $|s|>1$ are simple; at a pole of $B(\cdot)$ its residue is indicated by $b(\cdot) ; \phi_{0}(\cdot), \omega_{i}(\cdot)$, stand for residues of $\Phi_{0}(\cdot), \Omega_{i}(\cdot), i=1,2$, respectively. Note that $b(s)=0$ implies that $s$ is not a pole of $B(\cdot)$. From the set of relations (4.1-4.4), a set of relations for the residues is obtained; e.g., if $\tau$ is a pole of $B(\cdot)$ and also of $\Phi_{0}(\cdot)$, then it is seen from (1.4) that in general $\rho^{-}(\tau)$ is a pole of $\Omega_{2}(\cdot)$ and $r^{-}(\tau)$ is that of $\Omega_{1}(\cdot)$. The essential point of the analysis is to construct from the set of equations for the residues a nonnull solution such that $B(\cdot), \Phi_{0}, \Omega_{i}(\cdot), i=1,2$, are true meromorphic functions, i.e. the pole set of each of these functions has not a finite accumulation point. It turns out that there is a nontrivial solution to this problem. The solution of this problem is discussed in Sections 5, 6 and 7.

In Section 5 , it is shown that there exists a unique $T$ in $T>1$ such that the equations

$$
\begin{aligned}
& \frac{a_{1} T}{\rho^{-}(T)-T} b(T)+k_{1}\left(\rho^{-}(T), T\right) \phi_{0}(T)=0, h_{1}\left(\rho^{-}(T), T\right)=0, \\
& \frac{-a_{2} T}{r^{-}(T)-T} b(T)+k_{2}\left(r^{-}(T), T\right) \phi_{0}(T)=0, h_{2}\left(r^{-}(T), T\right)=0,
\end{aligned}
$$

for the residues $b(T)$ and $\phi_{0}(T)$ of $B(\cdot)$ and $\Phi_{0}(\cdot)$ possess a nonnull solution. Actually, $T$ is then determined by that zero in $T>1$, for which the main determinant of the set of equations (1.5) is zero. Note that (1.4) and (1.5) imply that $\rho^{-}(T)$ is not a pole of $\Omega_{2}(\cdot)$ and $r^{-}(T)$ not a pole of $\Omega_{1}(\cdot)$.

In Section 6 , it is shown that the set of nodes of the tree generated by $\tau_{0}^{(0)}=T$ is the pole set of $B(\cdot)$ as well as of $\Phi_{0}(\cdot)$. The poles of $\Omega_{i}(\cdot), i=1,2$, can be deduced from those of $B(\cdot)$ and $\Phi_{0}(\cdot)$. Further, a recursive set of equations is derived for the residues at these poles; see Lemmas 6.1 and 6.2 . In order to decide whether the pole set of $B(\cdot)$ can be used to define a meromorphic function information is required concerning the asymptotic behavior of the poles of $B(\cdot)$ and their residues $b(\cdot)$. This asymptotic behavior is studied in Section 7 . It is shown that this asymptotic behavior is such that for the pole set of $B(\cdot)$, a class of meromorphic functions can be constructed, similarly for $\Phi_{0}(\cdot), \Omega_{i}(\cdot), i=1,2$. The elements of these classes of meromorphic functions are parametrized by nonnegative integers $m_{b}, m_{\phi}, m_{i}, i=1,2$, bounded from below by $\widetilde{M}-1$, where $\widetilde{M}$ is defined by the asymptotic behavior of the residues, cf. (8.6).

In Section 8 , the meromorphic functions $\widetilde{B}(\cdot), \widetilde{\Phi}_{0}(\cdot), \widetilde{\Omega}_{i}(\cdot), i=1,2$, are introduced by using the pole sets obtained in Section 6 for the functions $B(\cdot), \Phi_{0}(\cdot)$, $\Omega_{i}(\cdot), i=1,2$; they contain the parameters $m_{b}, m_{\phi}, m_{i}, i=1,2$, cf. (8.2). Further, polynomials $\widehat{B}(s), \widehat{\Phi}_{0}(s), i=1,2$ are introduced; their degrees are indicated by $\widehat{N}_{b}$, $\widehat{N}_{\phi}, \widehat{N}_{i}, i=1,2$. With the functions so introduced, the functions

$$
\begin{gathered}
B(\cdot)=\widehat{B}(\cdot)+\widetilde{B}(\cdot), \Phi_{0}(\cdot)=\widehat{\Phi}(\cdot)+\widetilde{\Phi}(\cdot), \\
\Omega_{i}(\cdot)=\widehat{\Omega}(\cdot)+\widetilde{\Omega}_{i}(\cdot), \quad i=1,2
\end{gathered}
$$

are considered, cf. (8.7). These functions are substituted into equations (1.4), cf. (8.9). The asymptotic behavior at infinity of the relations so obtained is investigated. It leads to relations between $m$ and the degrees $\widehat{N}$ of the polynomials. Given $\tilde{M}$, appropriate choices of $m$.. determines the degrees $\hat{N}_{\ldots} .$. . With the degrees so determined, the functions (1.6) are again substituted in the equations (1.4). By considering the resulting equations for properly chosen zero-tuples of $h_{1}(\rho, \tau)$ and of 
$h_{2}(r, t)$, a system of linear, nonhomogeneous equations in unknown coefficients of the polynomials $\widehat{B}(\cdot), \widehat{\Phi}_{0}(\cdot), \widehat{\Omega}_{i}(\cdot), i=1,2$ is obtained. It is shown that this system has a unique solution, and so, the functions of (1.6) are all known and satisfy the equations; moreover, they are all regular in the closed unit disk centered at zero. Theorem 8.1 states that for $\widetilde{M} \geq 1, a_{1} \neq a_{2}$, the functions so constructed, whenever taking into account the norming condition, determine the bivariate generating functions of the stationary distribution uniquely if and only if $\frac{1}{a_{1}}+\frac{1}{a_{2}}>1$. The case $\tilde{M}=1$ is an important practical case. Then the construction of the solution for the case $\tilde{M}=2$ is discussed, whereas that for $\widetilde{M} \geq 3$ is exposed. In Remark 8.2 , the meaning of $T$ for the asymptotic behavior of the constructed solution is discussed.

In Section 9, the case $a_{1}=a_{2}, \pi_{1} \neq \pi_{2}$, is discussed. For this case, the construction of the solution of (1.4) is essentially simpler, because here the pole sets do not have a tree structure. In Section 10, relations are derived for some characteristic probabilities and moments of the model.

The approach developed in the present study is applicable to a larger class of asymmetrical two-server models, e.g., the asymmetrical variant of the model in [9]. This class is characterized by zero one-step transition probabilities to the north, north-east and east in the upper as well as in the lower triangle of the first quadrant [15].

\section{The Functional Equation}

The functional equations for the bivariate generating function of the joint distribution of the queue lengths $\mathbf{x}_{1}, \mathbf{x}_{2}$, of the asymmetrical shortest queue model have been derived in [1]; see Section III.1.2, p. 245. Below we recall these functional equations using mainly the same notation as in [1].

Denote for $|r| \leq 1$,

(ii) $\Omega_{2}(r):=\mathrm{E}\left\{r^{\mathbf{x}_{2}}\left(\mathbf{x}_{1}=0\right)\right\}$,

(iii) $\Omega_{1}(r):=\mathrm{E}\left\{r^{\mathbf{x}_{1}}\left(\mathbf{x}_{2}=0\right)\right\}$,

(iv) $B(r):=\Phi_{0}(r)\left(\frac{1}{a_{2}}+\pi_{1}\right)-\frac{1}{a_{2}} \Phi_{0}(0)-\left(1+\frac{1}{a_{1} r}\right) \mathrm{E}\left\{r^{\mathbf{x}_{1}}\left(\mathbf{x}_{1}=\mathbf{x}_{2}+1\right)\right\}$

$=-\Phi(r)\left(\frac{1}{a_{1}}+\pi_{2}\right)+\frac{1}{a_{1}} \Phi_{0}(0)+\left(1+\frac{1}{a_{2} r}\right) \mathrm{E}\left\{r^{\mathbf{x}_{2}}\left(\mathbf{x}_{2}=\mathbf{x}_{1}\right)\right\}$

$K_{1}\left(r_{1}, r_{2}\right):=r_{1}+\frac{1}{a_{1} r_{1}}+\frac{1}{a_{2} r_{2}}-\frac{b}{a_{1} a_{2}}$,

with

$K_{2}\left(r_{1}, r_{2}\right):=r_{2}+\frac{1}{a_{1} r_{1}}+\frac{1}{a_{2} r_{2}}-\frac{b}{a_{1} a_{2}}$

$$
\begin{aligned}
& a_{1}>0, a_{2}>0, b:=a_{1}+a_{2}+a_{1} a_{2}, \\
& 1>\pi_{1}>0,1>\pi_{2}>0, \pi_{1}+\pi_{2}+1, \\
& \pi_{1} \neq \pi_{2} \text { if } a_{1}=a_{2}, \text { cf. Remark } 2.3 \text { below. }
\end{aligned}
$$

The functional equations read: for $\left|r_{1}\right| \leq 1,\left|r_{2}\right| \leq 1$,

$$
\begin{aligned}
& \mathrm{E}\left\{r_{1}^{\mathbf{x}_{1}} r_{2}^{\mathbf{x}_{2}}\left(\mathbf{x}_{2}>\mathrm{x}_{1}\right)\right\} K_{1}\left(r_{1}, r_{2}\right)+\Omega_{2}\left(r_{2}\right) \frac{1}{a_{1}}\left(1-\frac{1}{r_{1}}\right) \\
& +\Phi_{0}\left(r_{1} r_{2}\right)\left[\pi_{2} r_{2}+\frac{1}{a_{1} r_{1}}-\frac{1}{a_{1}}-\pi_{2}\right]-B\left(r_{1} r_{2}\right)=0,
\end{aligned}
$$


(ii) $\quad \mathrm{E}\left\{r_{1}^{\mathbf{x}_{1}} r_{2}^{\mathbf{x}_{2}}\left(\mathbf{x}_{1}>\mathbf{x}_{2}\right)\right\} K_{2}\left(r_{1}, r_{2}\right)+\Omega_{1}(r) \frac{1}{a_{2}}\left(1-\frac{1}{r_{2}}\right)$

$$
+\Phi_{0}\left(r_{1} r_{2}\right)\left[\pi_{1} r_{1}+\frac{1}{a_{2} r_{2}}-\frac{1}{a_{2}}-\pi_{1}\right]+B\left(r_{1} r_{2}\right)=0 \text {. }
$$

From these equations it follows, by using the norming condition, i.e., $\operatorname{Pr}\left\{\mathbf{x}_{i} \geq 0, \mathbf{x}_{2} \geq 0\right\}=1$, that cf. [1], p. 242,

$$
\frac{1}{a_{1}} \operatorname{Pr}\left\{\mathbf{x}_{1}=0\right\}+\frac{1}{a_{2}} \operatorname{Pr}\left\{\mathbf{x}_{2}=0\right\}=\frac{1}{a_{1}}+\frac{1}{a_{2}}-1 \text {. }
$$

It follows that

$$
\frac{1}{a_{1}}+\frac{1}{a_{2}}>1
$$

is a necessary condition for the existence of a stationary distribution.

Remark 2.1: It will be shown, cf. Theorem 8.1, that $(2.6)$ is also a sufficient condition.

Assumption 2.1: In the present analysis it will be assumed that

$$
\frac{1}{a_{1}}+\frac{1}{a_{2}}>1
$$

For a zero-tuple $\left(\widehat{r}_{1}, \widehat{r}_{2}\right)$ of $K_{1}\left(r_{1}, r_{2}\right),\left|r_{1}\right| \leq 1,\left|r_{2}\right| \leq 1$, we have

$$
0<\mid \mathrm{E}\left\{\widehat{r}_{1} \mathbf{x}_{1} \widehat{r}_{2} \mathbf{x}_{2}\left(\mathbf{x}_{2}>\mathbf{x}_{1}\right) \mid \leq 1\right.
$$

and so it follows from $(2.4)(i)$,

$$
\Omega_{2}\left(\widehat{r}_{2}\right) \frac{1}{a_{2}}\left(1-\frac{1}{\widehat{r}_{1}}\right)+\Phi_{0}\left(\widehat{r}_{1} \widehat{r}_{2}\right)\left[\pi_{2} \widehat{r}_{2}+\frac{1}{a_{1} \widehat{r}_{1}}-\frac{1}{a_{1}}-\pi_{2}\right]-B\left(\widehat{r}_{1} \widehat{r}_{2}\right)=0 .
$$

Set

$$
\begin{gathered}
h_{1}(\rho, \tau):=a_{1} a_{2} \tau^{2}+\left(a_{1}-b \rho\right) \tau+a_{2} \rho^{2}, \\
h_{2}(r, t):=a_{1} a_{2} t^{2}+\left(a_{2}-b r\right) t+a_{1} r^{2}, \\
k_{1}(\rho, \tau):=-1+a_{2} \pi_{2}\left(a_{1} \tau-\rho\right), \\
k_{2}(r, t):=-1+a_{1} \pi_{1}\left(a_{2} t-r\right) .
\end{gathered}
$$

By using the properties of the zeros of $K_{1}\left(r_{1}, r_{2}\right)$ when simplifying the expression in square brackets in (2.7) and taking $\rho=\widehat{r}_{2}, \tau=\widehat{r}_{1} \widehat{r}_{2}$ it is readily verified that (2.7) is equivalent to

$$
\Omega_{2}(\rho)+\frac{a_{1} \tau}{\rho-\tau} B(\tau)+k_{1}(\rho, \tau) \Phi_{0}(\tau)=0
$$

for a zero-tuple $(\rho, \tau)$ of $h_{1}(\rho, \tau) ;|\rho| \leq 1,|\tau| \leq 1$;

$\Omega_{1}(r)-\frac{a_{2} t}{r-t} B(t)+k_{2}(r, t) \Phi_{0}(t)=0$,

for a zero-tuple $(r, t)$ of $h_{2}(r, t) ;|r| \leq 1,|t| \leq 1$;

note that the derivation of (2.9) (ii) is analogous to that of $(2.9)(i)$.

From the above, it is seen that the determination of the bivariate generating function $\mathrm{E}\left\{r_{1}^{\mathbf{x}_{1}} r_{2}^{\mathbf{x}_{2}}\right\}$ of the stationary joint distribution of the queue lengths requires the construction of functions $\Omega_{1}(\rho), \Omega_{2}(\rho), \Phi_{0}(\rho)$ and $B(\rho)$, which should satisfy the 
following conditions:

(i) they are regular for $|\rho|<1$ and the sum of the coefficients in their series expansions in powers of $\rho^{n}$ converges absolutely;

(ii) they satisfy relations (2.1) $(i v),(2.5),(2.9)(i),(i i)$ and, cf. $(2.1), \Omega_{i}(0)=$ $\Phi_{0}(0), i=1,2$.

Remark 2.2: Once functions $B(\cdot), \Phi_{0}(\cdot), \Omega_{1}(\cdot)$ and $\Omega_{2}(\cdot)$ have been constructed, which are not identically zero and satisfy conditions (2.10), so that $\mathrm{E}\left\{r_{1}^{\mathbf{x}_{1}} r_{2}^{\mathbf{x}_{2}}\right\}$, $\left|r_{1}\right| \leq 1,\left|r_{2}\right| \leq 1$, is then determined via (2.4), then it follows that the Kolmogorov equations for the stationary probabilities possess an absolute convergent nonnull solution. By applying the well known Foster criterion, cf. [3], p. 25, it follows that the queue length process $\left(\mathbf{x}_{1}(t), \mathbf{x}_{2}(t)\right)$, of which the state space is irreducible, is positive recurrent and further that there is only one solution, which satisfies (2.10) and the norming condition. Hence it suffices to construct functions $B(\cdot), \Omega_{1}(\cdot)$ and $\Omega_{1}(\cdot)$, which satisfy $(2.10)$.

Remark 2.3: The analysis of the problem formulated in (2.10) for the case $a_{1}=$ $a_{2}, \pi_{1} \neq \pi_{2}$, differs from that for the case $a_{1} \neq a_{2}$. The analysis in Sections 6,7 and 8 concerns the case $a_{1} \neq a_{2}$. In Section 9 , the case $a_{1}=a_{2}, \pi_{1} \neq \pi_{2}$ is discussed. For the case $a_{1}=a_{2}, \pi_{1}=\pi_{2}$, see [12].

\section{On the Zeros of $h_{1}(\rho, \tau)$ and $h_{2}(r, t)$}

In this section we shall describe several properties of the zeros of $h_{1}(\rho, \tau)$ and $h_{2}(r, t)$ and introduce several functions of these zeros; these functions are needed to describe the functions $\Omega_{1}(r), \Omega_{2}(\rho), \Phi_{0}(\tau)$ and $B(\tau)$. Because of the symmetry between $h_{1}(\rho, \tau)$ and $h_{2}(r, t)$, we mainly restrict the discussion to $h_{1}(\rho, \tau)$; those for $h_{2}(r, t)$ follow by interchanging $a_{1}$ and $a_{2}$.

From (2.8) we have

$$
\begin{aligned}
& h_{1}(\rho, \tau)=0 \Rightarrow \tau(\rho)=\frac{1}{2 a_{1} a_{2}}\left[-a_{1}+b \rho \pm \sqrt{\left(a_{1}-b \rho\right)^{2}-4 a_{1} a_{2}^{2} \rho^{2}}\right] \\
& h_{1}(\rho, \tau)=0 \Rightarrow \rho(\tau)=\frac{1}{2 a_{2}}\left[b \tau \pm \sqrt{b^{2} \tau^{2}-4 a_{1} a_{2}\left(\tau+a_{2} \tau^{2}\right)}\right] .
\end{aligned}
$$

Lemma 3.1: For every $\rho$ with $|\rho| \geq 1, \rho \neq 1$, the two zeros $\tau^{ \pm}(\rho)$ of $h_{1}(\rho, \tau)$ may be defined so that

$$
\left|\tau^{-}(\rho)\right|<|\rho|<\left|\tau^{+}(\rho)\right|
$$

and, similarly, for the two zeros $\rho(\tau)$ with $|\tau| \geq 1, \tau \neq 1$, i.e.,

$$
\left|\rho^{-}(\tau)\right|<|\tau|<\left|\rho^{+}(\tau)\right| \text {. }
$$

Analogously, for $h_{2}(r, t)=0$,

$$
\begin{gathered}
\left|t^{-}(r)\right|<|r|<\left|t^{+}(r)\right| \text { for }|r| \geq 1, r \neq 1 \\
\left|r^{-}(t)\right|<|t|<\left|r^{+}(t)\right| \text { for }|t| \geq 1, t \neq 1 .
\end{gathered}
$$

For the proof of Lemma 3.1, see Appendix A.

From (3.1), it is seen that $\tau(\rho)$ has two branch points $\rho^{-}$and $\rho^{+}$and that $\rho(\tau)$ 
has also two branch points $\tau^{-}$and $\tau^{+}$. It is readily verified that

$$
\begin{gathered}
\rho^{ \pm}=\left[1+\frac{a_{2}}{a_{1}}\left(1 \pm \sqrt{a_{1}}\right)^{2}\right]^{-1}, \quad 0<\rho^{-}<\rho^{+} \leq 1, \\
\tau^{-}=0, \quad 0<\frac{4 a_{1} a_{2}}{b^{2}-4 a_{1} a_{2}^{2}}=\tau^{+}<1 ;
\end{gathered}
$$

analogous relations hold for the branch points $r^{ \pm}$and $t^{ \pm}$of the zeros of $h_{2}(r, t)$. by

For real $\rho$ and $\tau$, the curve $h_{1}(\rho, \tau)=0$ is a hyperbola with center $\left(\check{\rho}_{m}, \check{\tau}_{m}\right)$ given

$$
\check{\rho}_{m}=\frac{a_{1} b}{b^{2}-4 a_{1} a_{2}^{2}}>0, \quad \check{\tau}_{m}=\frac{2 a_{1} a_{2}}{b^{2}-4 a_{1} a_{2}^{2}}>0,
$$

and asymptotes given by

$$
\rho-\check{\rho}_{m}=\frac{1}{2 a_{2}}\left[b \pm \sqrt{b^{2}-4 a_{1} a_{2}^{2}}\right]\left(\tau-\check{\tau}_{m}\right) .
$$

Some special zeros of $h_{1}(\rho, \tau)$ are listed below:

$$
\begin{gathered}
\tau^{+}(0)=-\frac{1}{a_{2}}, \quad \tau^{-}(0)=0, \quad \rho^{ \pm}(0)=0, \\
\tau^{-}(1)=\min \left(1, \frac{1}{a_{1}}\right), \quad \tau^{+}(1)=\max \left(1, \frac{1}{a_{1}}\right), \\
\rho^{-}(1)=\min \left(1, a_{1}+\frac{a_{1}}{a_{2}}\right), \quad \rho^{+}(1)=\max \left(1, a_{1}+\frac{a_{1}}{a_{2}}\right), \\
\frac{d \rho(\tau)}{d \tau}=\frac{a_{2}\left(a_{1}-1\right)}{a_{1} a_{2}+a_{1}-a_{2}} \text { for }\left(\rho(\tau, \tau)=(1,1) \text { and } a_{2} a_{1}+a_{2}-1 \neq 0 .\right.
\end{gathered}
$$

In Figure 3.1, we depicted the hyperbola $h_{1}(\rho, \tau)=0$ and $\tau=0$ being its tangent line at $\rho=0, \tau=0$.

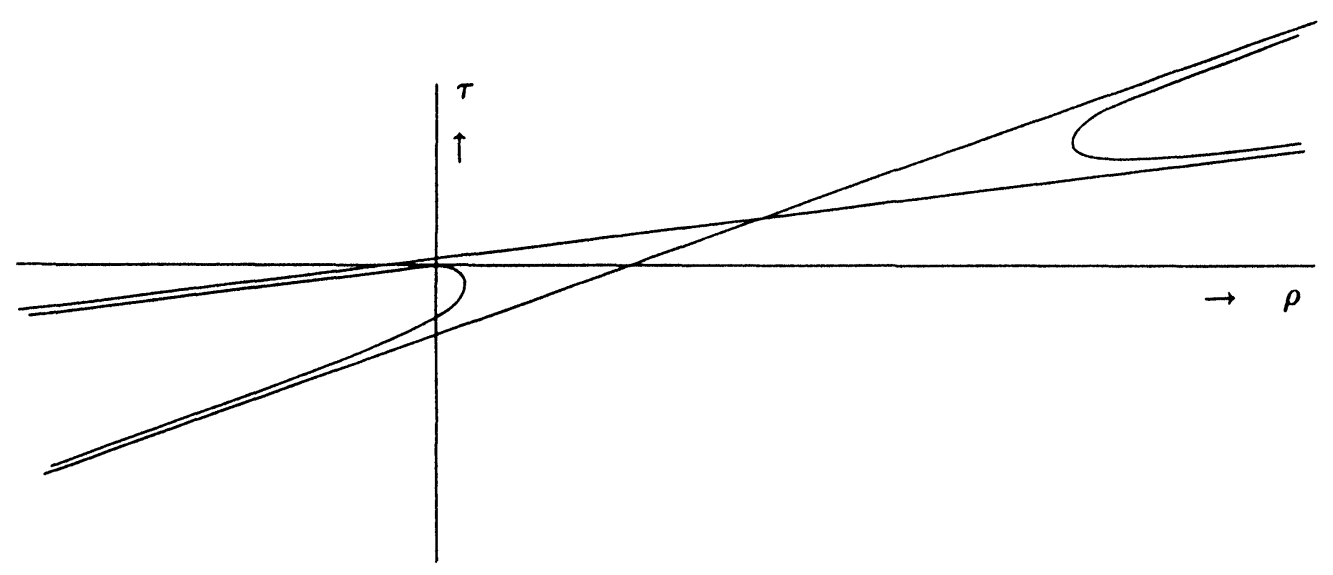

Figure 3.1

Let $\left(\rho_{n}, \tau_{n}\right)$ be a zero-tuple of $h_{1}(\rho, \tau)$ with $\rho_{n}=\rho^{-}\left(\tau_{n}\right), \tau_{n}=\tau^{+}\left(\rho_{n}\right)$, and $\tau_{n}>1$; see Figure 3.2 . 


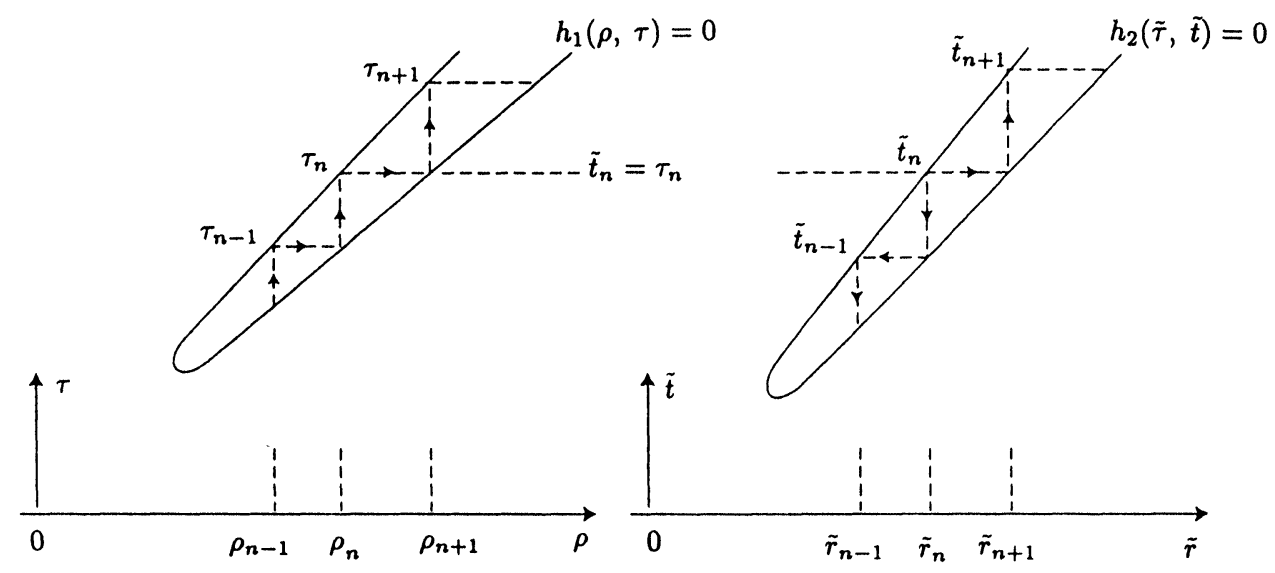

Figure 3.2

Starting from $\left(\rho_{n}, \tau_{n}\right)$, we construct a series of zero-tuples of $h_{1}(\rho, \tau)$, cf. (3.2) and $(3.3)$ :

$$
\left(\rho_{\nu}, \tau_{\nu}\right), \ldots,\left(\rho_{n-1}, \tau_{n-1}\right),\left(\rho_{n}, \tau_{n}\right),\left(\rho_{n+1}, \tau_{n+1}\right), \ldots
$$

They are recursively defined by:

$$
\begin{gathered}
\tau_{n-1}=\tau^{-}\left(\rho_{n}\right), \quad \rho_{n+1}=\rho^{+}\left(\tau_{n}\right), \\
\rho_{n-1}=\rho^{-}\left(\tau_{n-1}\right), \quad \tau_{n+1}=\tau^{+}\left(\rho_{n+1}\right) .
\end{gathered}
$$

The sequence in (3.8) will be called the ladder generated by $\tau_{n}$ on the hyperbola $h_{1}(\rho, \tau)$. Its "up-ladder" is unbounded, while its "down-ladder" is finite and stopped at that index $\nu$, for which $0<\tau_{\nu}<1$ or $\rho_{\nu}<1$.

Whenever $a_{1} \neq a_{2}$, cf. Remark 2.1, the zero-tuple $\left(\rho_{n}, \tau_{n}\right)$ of $h_{1}(\rho, \tau)$ induces also a ladder viz.

$$
, \ldots,\left(\tilde{r}_{n-1}, \tilde{t}_{n-1}\right),\left(\widetilde{r}_{n}, \tilde{t}_{n}\right),\left(\widetilde{r}_{n+1}, \tilde{\tau}_{n+1}\right), \ldots
$$

on the hyperbola $h_{2}(r, t)=0$, and $\tilde{t}_{n \mp i} \neq \tau_{n \mp i}, i \neq 0$. It is recursively defined by

$$
\begin{gathered}
\tilde{t}_{n}=\tau_{n}, \quad \tilde{r}_{n}=r^{-}\left(\tilde{t}_{n}\right), \\
\tilde{t}_{n-1}=t^{-}\left(\tilde{r}_{n}\right), \quad \tilde{r}_{n+1}=r^{+}\left(\tilde{t}_{n}\right), \\
\tilde{r}_{n-1}=r^{-}\left(\tilde{t}_{n-1}\right), \quad \tilde{t}_{n+1}=t^{+}\left(\tilde{r}_{n+1}\right) ;
\end{gathered}
$$

again the "down-ladder" is stopped at that $\mu$ for which $0<\tilde{t}_{\mu}<1$ or $0<\widetilde{r}_{\mu}<1$.

Remark 3.1: Note that for $a_{1} \neq a_{2}$, i.e., $h_{1}(\rho, t) \not \equiv h_{2}(\rho, t)$,

$$
\begin{array}{ll}
\tilde{t}_{n-1} \neq \tau_{n-1}, & \tilde{t}_{n+1} \neq \tau_{n+1}, \\
\tilde{r}_{n-1} \neq r_{n-1}, & \tilde{r}_{n+1} \neq r_{n+1} .
\end{array}
$$

Analogously, a zero-tuple of $h_{2}(r, t)$ with $r_{n}=r^{-}\left(t_{n}\right), t_{n}=t^{+}\left(r_{n}\right)$ and $t_{n}>1$ or $r_{n}>1$ generates a ladder on $h_{2}(r, t)=0$ and induces a ladder on $h_{1}(\tilde{\rho}, \tilde{t})=0$; see Figure 3.3. 


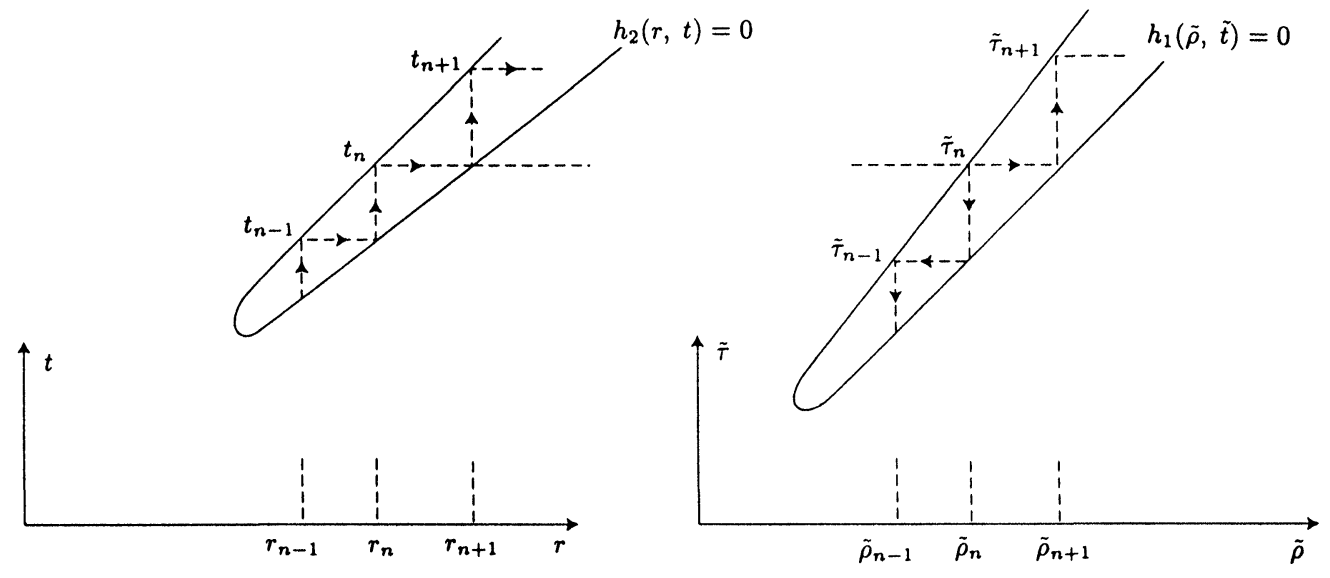

Figure 3.3

These ladders are defined analogously to those in Figure 3.2. Actually, interchange in (3.9) and (3.11) $\rho$ and $r$ and also $\tau$ and $t$. It should be noted that the ladder in (3.8) with top $\left(\rho_{n}, \tau_{n}\right)$ is identical to the ladder generated on $h_{1}(\rho, \tau)=0$ with top $\left(\rho_{n+1}, \tau_{n+1}\right)$ if $\tau_{n+1}=\tau^{+}\left(\rho_{n+1}\right), \rho_{n+1}=\rho^{+}\left(\tau_{n}\right)$.

However, the ladder on $h_{2}(r, t)$ induced by $\left(\rho_{n}, \tau_{n}\right)$ generally differs from that induced on $h_{2}(r, t)$ by $\left(\rho_{n+1}, \tau_{n+1}\right)$.

We shall denote by

(i) $\quad l\left(\tau_{n}\right)$ with $h_{1}\left(\rho_{n}, \tau_{n}\right)=0$, the ladder generated by $\left(\rho_{n}, \tau_{n}\right)$ on $h_{1}(\rho, \tau)=0$;

(ii) $\tilde{l}\left(\tau_{n}\right)$ the ladder induced by $\left(\rho_{n}, \tau_{n}\right)$ on $h_{2}(\tilde{r}, \tilde{t})=0$ with $h_{1}\left(\rho_{n}, \tau_{n}\right)=0$.

Analogously, we define the ladders $l\left(t_{n}\right)$ and $\tilde{l}\left(t_{n}\right)$.

Remark 3.2: Note that every point of $h_{1}(\rho, \tau)$ with $\rho>1$ or $\tau>1$ induces a ladder on $h_{2}(r, t)$ and visa versa.

Further, it is readily seen from (3.9) and (3.10) that, cf. Figure 3.2 and 3.3:

$$
\begin{gathered}
\tau_{n+1}=\tau^{+}\left(\rho^{+}\left(\tau_{n}\right)\right), \quad \rho_{n+1}=\rho^{+}\left(\tau^{+}\left(\tau^{+}\left(\rho_{n}\right)\right),\right. \\
t_{n+1}=t^{+}\left(r^{+}\left(t_{n}\right)\right), \quad r_{n+1}=r^{+}\left(t^{+}\left(r_{n}\right)\right), \\
\tau_{n+m} \rightarrow \infty, \rho_{n+m} \rightarrow \infty, t_{n+m} \rightarrow \infty, r_{n+m} \rightarrow \infty, \\
\text { for } m \rightarrow \infty \text { and } \tau_{n}>1, t_{n}>1 .
\end{gathered}
$$

Next we introduce a notation to describe all the ladder points on the up-ladders on $h_{1}(\rho, t)=0$ as well as on $h_{2}(r, t)=0$ generated by a point

$$
\left(\rho_{0}, \tau_{0}\right) \text { of } h_{1}(\rho, \tau)=0 .
$$

Define for $m=0,1,2, \ldots$, the binary numbers

$$
\begin{gathered}
\delta_{m}:=\delta_{m 1} \delta_{m 2} \ldots \delta_{m m} \text { with } \delta_{m j} \in\{0,1\}, \quad j=1, \ldots, m, \\
\mathscr{B}_{m}:=\left\{\delta: \delta=\delta_{j}, j=0, \ldots, 2^{m}-1\right\} \equiv\left\{\delta: \delta \in\left\{0,1,2, \ldots, 2^{m}-1\right\}\right\} .
\end{gathered}
$$


The tree generated by $\left(\tau_{0}, \rho_{0}\right)$ is defined as follows:

its nodes at the $n$th level, $n=0,1, \ldots$, are

(i) $\quad \tau_{\delta}^{(n)}(n)$ with $\delta_{j}^{(n)}<\delta_{j+1}^{(n)}, \delta_{j}^{(n)}, \delta_{j+1}^{(n)} \in \mathfrak{B}_{n}, \quad j=0, \ldots, 2^{n-1}$,

(ii) $\quad \tau_{\delta_{j}^{(n+1)}}^{(n+1)}:=\tau^{+}\left(\rho^{+}\left(\tau_{\delta_{j}^{(n)}}^{(n)}\right)\right)$ for $\delta_{j}^{(n+1)}=2 \delta_{j}^{(n)}$,

(iii) $\tau_{0}^{(0)} \quad=\tau_{0}=\tau^{+}\left(\rho_{0}\right)$.

$$
:=t^{+}\left(r^{+}\left(\tau_{\delta_{j}^{(n)}}^{(n)}\right)\right) \text { for } \delta_{j}^{(n+1)}=2 \delta_{j}^{(n)}+1 .
$$

In Figure 3.4, we depicted the nodes at levels $0,1,2$ and 3 for the tree generated by $\left(\tau_{0}, \rho_{0}\right)$.

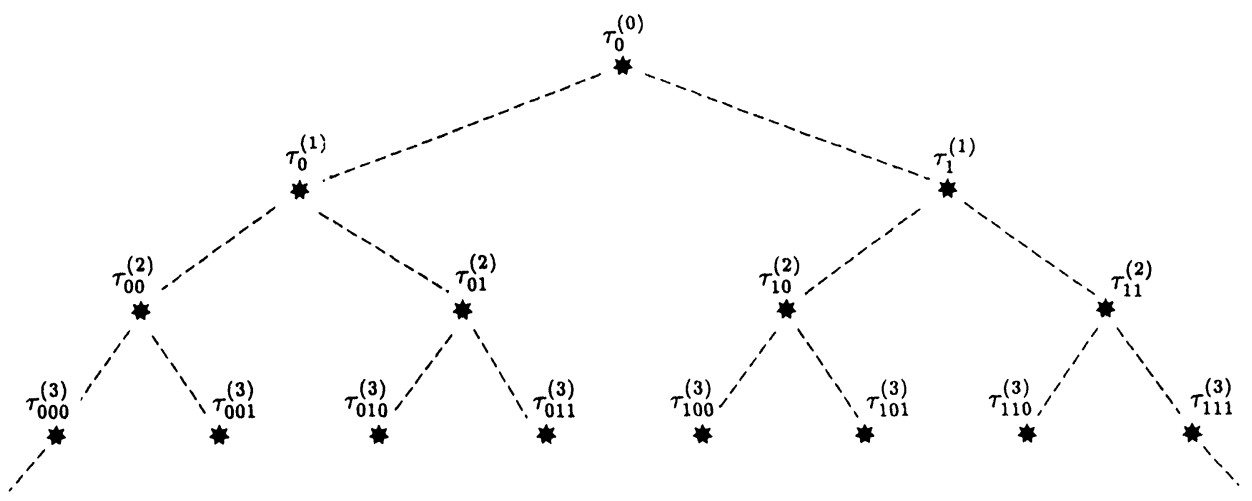

Figure 3.4

From the definitions above it is readily seen that the tree so constructed contains all the ladder points on $h_{1}(\rho, \tau)=0$ and $h_{2}(r, t)=0$ generated by $\tau_{0}$ with $h_{1}\left(\rho_{0}, \tau_{0}\right)=0$; note that $l\left(\tau_{0}\right)$ is the set of nodes on the left branch of the tree and $\tilde{l}\left(\tau_{0}\right)$ is the set of nodes on the right branch of the tree.

The tree generated by $\left(t_{0}, r_{0}\right)$ is defined analogously, interchange the symbols $\tau$ and $t$ and also $\rho$ and $r$.

We conclude this section with the derivation of some asymptotic results. From $(3.1)$, it is seen that

$$
\begin{gathered}
R_{1}^{ \pm}:=\lim _{r \rightarrow \infty} \frac{\rho^{ \pm}(\tau)}{\tau}=\frac{1}{2 a_{2}}\left(b \pm d_{1}\right), \\
R_{2}^{ \pm}:=\lim _{t \rightarrow \infty} \frac{r^{ \pm}(t)}{t}=\frac{1}{2 a_{1}}\left(b \pm d_{2}\right), \\
T_{1}^{ \pm}:=\lim _{\rho \rightarrow \infty} \frac{\tau^{ \pm}(\rho)}{\rho}=\frac{1}{2 a_{1} a_{2}}\left(b \pm d_{1}\right), \\
T_{2}^{ \pm}:=\lim _{r \rightarrow \infty} \frac{t^{ \pm}(r)}{r}=\frac{1}{2 a_{1} a_{2}}\left(b \pm d_{2}\right),
\end{gathered}
$$

with 


$$
d_{1}:=\sqrt{b^{2}-4 a_{1} a_{2}^{2}}, \quad d_{2}:=\sqrt{b^{2}-4 a_{1}^{2} a_{2}}
$$

Note that, cf. Lemma 3.1 , for $j=1,2$,

$$
\begin{gathered}
R_{j}^{+}>1, T_{j}^{+}>1, \quad R_{j}^{ \pm} T_{j}^{\mp}=1, \\
a_{1} a_{2}\left(T_{1}^{ \pm}\right)^{2}-b T_{1}^{ \pm}+a_{2}=0, \quad a_{2}\left(R_{1}^{ \pm}\right)^{2}-b R_{1}^{ \pm}+a_{1} a_{2}=0 \\
a_{2}\left(R_{1}^{ \pm}-1\right)^{2}-\left(b-2 a_{2}\right)\left(R_{1}^{ \pm}-1\right)-a_{1}=0 .
\end{gathered}
$$

Further, from (2.4) by using (3.16) and (3.17),

$$
\begin{gathered}
\lim _{\rho \rightarrow \infty} \frac{d \tau^{ \pm}(\rho)}{d \rho}=\frac{b T_{1}^{ \pm}-2 a_{2}}{2 a_{1} a_{2} T_{1}^{ \pm}-b}=\left[R_{1}^{\mp}\right]^{-1}, \\
\lim _{r \rightarrow \infty} \frac{d \rho^{ \pm}(\tau)}{d \tau}=\frac{2 a_{1} a_{2}-b R_{1}^{ \pm}}{b-2 a_{2} R_{1}^{ \pm}}=T_{1}^{\mp} .
\end{gathered}
$$

Because

we have

$$
R_{1}^{-}<1<R_{1}^{+} \text {and } R_{1}^{-} R_{1}^{+}=a_{1}
$$

and

$$
R_{1}^{-}<\min \left(1, a_{1}\right), R_{1}^{+}>\max \left(1, a_{1}\right)
$$

\section{Meromorphic Continuation}

In this section we shall consider the meromorphic continuation of the functions $\Omega_{1}(s)$, $\Omega_{2}(s), B(s)$ and $\Phi_{0}(s)$ out from $|s| \leq 1$ into $|s|>1$.

Lemma 4.1: The functions $\Omega_{1}(s), \Omega_{2}(s), B(s)$ and $\Phi_{0}(s)$ can be continued meromorphically out from $|s| \leq 1$ into $|s|>1$.

For the proof of this lemma, see Appendix B.

Remark 4.1: The lemma does not imply that these continuations are meromorphic functions, i.e., have only a finite number of poles in every finite domain, but it implies that their only singularities are poles or accumulation points of poles.

Assumption 4.1: Henceforth it will be assumed that the meromorphic continuations of $B(\cdot), \Phi_{0}(\cdot), \Omega_{1}(\cdot)$ and $\Omega_{2}(\cdot)$ are all meromorphic functions.

Remark 4.2: Whenever Assumption 4.1 leads to the construction of functions $B(\cdot), \Phi_{0}(\cdot), \Omega_{1}(\cdot)$ and $\Omega_{2}(\cdot)$, which satisfy conditions $(2.10)$, our problem is solved, because there exists only one set of such functions, cf. Remark 2.2.

The meromorphic continuations of $B(\cdot), \Phi_{0}(\cdot), \Omega_{1}(\cdot)$ and $\Omega_{2}(\cdot)$ will be indicated by the same symbols, respectively.

It is further shown in Appendix $\mathrm{B}$ that the functional equations (2.9) can be extended into the domains $|\tau|>1,|\rho|>1,|t|>1$ and $|r|>1$. Actually it is shown that the following relations hold for all those $\tau, \rho, r, t$ for which $B(\cdot), \Phi_{0}(\cdot)$, $\Omega_{1}(\cdot), \Omega_{2}(\cdot)$ are finite:

$$
\Omega_{2}(\rho)+\frac{a_{1} \tau^{ \pm}(\rho)}{\rho-\tau^{ \pm}(\rho)} B\left(\tau^{ \pm}(\rho)\right)+k_{1}\left(\rho, \tau^{ \pm}(\rho)\right) \Phi_{0}\left(\tau^{ \pm}(\rho)\right)=0
$$




$$
\begin{gathered}
\text { for } h_{1}\left(\rho, \tau^{ \pm}(\rho)\right)=0, \\
\Omega_{1}(r)-\frac{a_{2} t^{ \pm}(r)}{r-t^{ \pm}(r)} B\left(t^{ \pm}(r)\right)+k_{2}\left(r, t^{ \pm}(r)\right) \Phi_{0}\left(t^{ \pm}(r)\right)=0 \\
\text { for } h_{2}\left(r, t^{ \pm}(r)\right)=0, \\
\Omega_{2}\left(\rho^{ \pm}(\tau)\right)+\frac{a_{1} \tau}{\rho^{ \pm}(\tau)-\tau} B(\tau)+k_{1}\left(\rho^{ \pm}(\tau), \tau\right) \Phi_{0}(\tau)=0 \\
\text { for } h_{1}\left(\rho^{ \pm}(\tau), \tau\right)=0, \\
\Omega_{1}\left(r^{ \pm}(t)\right)-\frac{a_{2} t}{r^{ \pm}(t)-t} B(t)+k_{2}\left(r^{ \pm}(t), t\right) \Phi_{0}(t)=0 \\
\text { for } h_{2}\left(r^{ \pm}(t), t\right)=0 .
\end{gathered}
$$

Remark 4.3: From the analysis in Appendix B it is seen that the relations (4.1)(4.4), are independent of Assumption 4.1. Note that (4.2) and (4.4), and similarly, (4.1) and (4.3), are not independent.

Assumption 4.2: Henceforth, it will be assumed that the poles of $B(\cdot), \Phi_{0}(\cdot)$, $\Omega_{1}(\cdot), \Omega_{2}(\cdot)$ are all simple.

Remark 4.4: Concerning the introduction of the latter assumption, it is noted that Remark 4.2 also applies here.

Put for finite $t$ and $\rho$ :

$$
\begin{gathered}
b(t):=\lim _{s \rightarrow t}(s-t) B(s), \quad \phi_{0}(t):=\lim _{s \rightarrow t}(s-t) \Phi_{0}(s), \\
\omega_{i}(\rho):=\lim _{r \rightarrow \rho}(r-\rho) \Omega_{i}(r), \quad i=1,2 .
\end{gathered}
$$

Assumption 4.2 implies that these limits exist. Obviously, cf. Assumption 4.2,

$$
\begin{gathered}
|b(t)| \neq 0 \Leftrightarrow t \text { is a pole of } B(\cdot), \\
\left|\phi_{0}(t)\right| \neq 0 \Leftrightarrow t \text { is a pole of } \Phi_{0}(\cdot), \\
\left|\omega_{i}(\rho)\right| \neq 0 \Leftrightarrow \rho \text { is a pole of } \Omega_{i}(\cdot), \quad i=1,2 .
\end{gathered}
$$

Note that (2.10) implies that

$$
\begin{gathered}
b(t)=0 \text { and } \phi_{0}(t)=0 \text { for }|t| \leq 1, \\
\omega_{i}(\rho)=0 \text { for }|\rho| \leq 1, i=1,2 .
\end{gathered}
$$

From (4.1)-(4.6), it readily follows that, cf. (3.4),

for $|\rho| \neq \rho^{ \pm}$and $h_{1}\left(\rho, \tau^{ \pm}(\rho)\right)=0$,

$\omega_{2}(\rho)+\left[\frac{a_{1} \tau^{ \pm}(\rho)}{\rho-\tau^{ \pm}(\rho)} b\left(\tau^{ \pm}(\rho)\right)+k_{1}\left(\rho, \tau^{ \pm}(\rho)\right) \phi_{0}\left(\tau^{ \pm}(\rho)\right)\right]\left[\frac{d \tau^{ \pm}(\sigma)}{d \sigma}\right]_{\sigma=\rho}^{-1}=0$

for $|r| \neq r^{ \pm}$and $h_{2}^{ \pm}\left(r, t^{ \pm}(r)\right)=0$,

$\omega_{1}(r)+\left[\frac{-a_{2} t^{ \pm}(r)}{r-t^{ \pm}(r)} b\left(t^{ \pm}(r)\right)+k_{2}\left(r, t^{ \pm}(r)\right) \phi_{0}\left(t^{ \pm}(r)\right)\right]\left[\frac{d t^{ \pm}(\sigma)}{d \sigma}\right]_{\sigma=r}^{-1}=0$ 
for $|\tau| \neq \tau^{ \pm}$and $h_{1}\left(\rho^{ \pm}(\tau), \tau\right)=0$,

$$
\omega_{2}\left(\rho^{ \pm}(\tau)\right)\left[\frac{d \rho^{ \pm}(\sigma)}{d \sigma}\right]_{\sigma=r}^{-1}+\frac{a_{1} \tau}{\rho^{ \pm}(\tau)-\tau} b(\tau)+k_{1}\left(\rho^{ \pm}(\tau), \tau\right) \phi_{0}(\tau)=0 ;
$$

for $|t| \neq t^{ \pm}$and $h_{2}\left(r^{ \pm}(t), t\right)=0$

$$
\omega_{1}\left(r^{ \pm}(t)\right)\left[\frac{d r^{ \pm}(\sigma)}{d \sigma}\right]_{\sigma=r}^{-1}+\frac{-a_{2} t}{r^{ \pm}(t)-t} b(t)+k_{2}\left(r^{ \pm}(t), t\right) \phi_{0}(t)=0 .
$$

In Sections 6, 7, 8 and 9, relations (4.8)-(4.11) will be used to calculate the residues of $B(\cdot), \Phi_{0}(\cdot), \Omega_{1}(\cdot), \Omega_{2}(\cdot)$ at their various poles. Note that relations $(4.10)$ and (4.11) on the one hand and (4.8) and (4.9) on the other hand are dependent on each other.

\section{The Equation for the Top of the Tree}

In this section, we derive a relation for the smallest in absolute value pole of $B(\cdot)$ and of $\Phi_{0}(\cdot)$.

From (4.3) and (4.4) we have that for $|\tau|>1, \quad h_{1}\left(\rho^{-}(\tau), \tau\right)=0$, $h_{2}\left(r^{-}(\tau), \tau\right)=0$ :

$$
\begin{aligned}
& \frac{a_{1} \tau}{\rho^{-}(\tau)-\tau} B(\tau)+k_{1}\left(\rho^{-}(\tau), \tau\right) \Phi_{0}(\tau)=-\Omega_{2}\left(\rho^{-}(\tau)\right) \\
& \frac{-a_{2} \tau}{r^{-}(\tau)-\tau} B(\tau)+k_{2}\left(r^{-}(\tau), \tau\right) \Phi_{0}(\tau)=-\Omega_{1}\left(r^{-}(\tau)\right)
\end{aligned}
$$

Put

$$
D(\rho(\tau), r(\tau), \tau):=\left|\begin{array}{cc}
\frac{a_{1} \tau}{\rho(\tau)-\tau} & k_{1}(\rho(\tau), \tau) \\
\frac{-a_{2} \tau}{r(\tau)-\tau} & k_{2}(r(\tau), \tau)
\end{array}\right|
$$

A simple calculation using (2.3), (2.8) and (5.3) shows that

$$
\begin{gathered}
\frac{1}{\tau}\left(\rho^{-}(\tau)-\tau\right)\left(r^{-}(\tau)-\tau\right) D\left(\rho^{-}(\tau), r^{-}(\tau), \tau\right) \\
=\left[b-a_{1} a_{2}\left(\pi_{1} r^{-}(\tau)+\pi_{2} \rho^{-}(\tau)\right)\right] \tau-a_{1} r^{-}(\tau)-a_{2} \rho^{-}(\tau) .
\end{gathered}
$$

Because $\rho^{-}(\tau)-\tau \neq 0, r^{-}(\tau)-\tau \neq 0$ for $|\tau|>1$, it follows that

$$
D\left(\rho^{-}(\tau), r^{-}(\tau), \tau\right)=0 \Leftrightarrow \tau=\frac{a_{1} r^{-}(\tau)+a_{2} \rho^{-}(\tau)}{b-a_{1} a_{2}\left[\pi_{1} r^{-}(\tau)+\pi_{2} \rho^{-}(\tau)\right]} .
$$

From (2.3) and (3.7), it is seen that

$$
\frac{b}{a_{1} a_{2}}-\pi_{1} r^{-}(1)-\pi_{2} \rho^{-}(1)>0 .
$$




\section{Lemma 5.1:}

(i) The equation

$$
\tau=\frac{\frac{r^{-}(\tau)}{a_{2}}+\frac{\rho^{-}(\tau)}{a_{1}}}{\frac{b}{a_{1} a_{2}}-\left\{\pi_{1} r^{-}(\tau)+\pi_{2} \rho^{-}(\tau)\right\}}
$$

has in $\tau>1$ a unique root $\tau=T$, of multiplicity one,

(ii) for $\frac{1}{a_{1}}+\frac{1}{a_{2}}>1$, it is given by

$$
T=\left(\frac{1}{a_{1}}+\frac{1}{a_{2}}\right)^{2}
$$

furthermore,

$$
\rho^{-}(T)=r^{-}(T)=\frac{1}{a_{1}}+\frac{1}{a_{2}} .
$$

Proof: It is readily seen that the above equation is equivalent to

$$
\tau=\frac{1+\frac{1}{a_{2}}\left(1-\frac{r^{-}(\tau)}{\tau}\right)+\frac{1}{a_{1}}\left(1-\frac{\rho^{-}(\tau)}{\tau}\right)}{1-\pi_{1}\left(1-\frac{r^{-}(\tau)}{\tau}\right)-\pi_{2}\left(1-\frac{\rho^{-}(\tau)}{\tau}\right)} .
$$

Because (cf. Lemma 3.1 and (3.16)),

$$
\begin{aligned}
& 0<\frac{r^{-}(\tau)}{\tau}<1, \quad 0<\frac{\rho^{-}(\tau)}{\tau}<1 \text { for } \tau>1, \\
& \frac{r^{-}(\tau)}{\tau} \downarrow R_{2}^{-}, \quad \frac{\rho^{-}(\tau)}{\tau} \downarrow R_{1}^{-1} \text { for } \tau: 1 \rightarrow \infty
\end{aligned}
$$

and because the right-hand side of (5.7) is readily seen to be larger than 1 for $\tau \geq 1$ and it is continuous and increasing in $\tau$ with a finite limit for $\tau \rightarrow \infty$, the first statement of the lemma follows. It is simply verified that $\tau=T, \rho^{-}(\tau)=\rho^{-}(T)$, $r^{-}(\tau)=r^{-}(T)$ satisfy (5.7). It remains to show that, cf. (3.1) (ii) and Lemma 3.1,

(i) $\quad \rho^{-}(T)<T, r^{-}(T)<T$,

(ii) $\quad h_{1}\left(\rho^{-}(T), T\right)=0, h_{2}\left(r^{-}(T), T\right)=0$.

Because $\frac{1}{a_{1}}+\frac{1}{a_{2}}>1,(5.8)(i)$ follows. Further by noticing that $T=[\rho-(T)]^{2}$, it is readily seen that (5.8) (ii) holds.

Assumption 5.1: Henceforth, it will be assumed that, cf. also Assumption 6.1.

$$
\left|\Omega_{2}\left(\rho^{-}(T)\right)\right|<\infty,\left|\Omega_{1}\left(r^{-}(T)\right)\right|<\infty
$$

Remark 5.1: Concerning the introduction of the latter assumption it is noted that Remark 4.2 also applies here.

Since $\tau=T$ is a simple zero of the determinant of (5.3), it follows from (5.2), and Assumption 5.1 that

$$
\tau=T \text { is a simple pole of } B(\tau) \text { and also of } \Phi_{0}(\tau) \text {. }
$$

In Section 8, Remark 8.2, it will be shown that $T$ is the smallest pole of $B(\cdot)$ and also of $\Phi_{0}(\cdot)$.

From (4.6), (5.2) and Assumption 5.1, we obtain, 


$$
\begin{aligned}
& \frac{a_{1} T}{\rho^{-}(T)-T} b(T)+k_{1}\left(\rho^{-}(T), T\right) \phi_{0}(T)=0 \\
& \frac{-a_{2} T}{r^{-}(T)-T} b(T)+k_{2}\left(r^{-}(T), T\right) \phi_{0}(T)=0
\end{aligned}
$$

note that these relations are linearly dependent.

Remark 5.2: A simple calculation shows that for $\tau>1$ the determinant

Similarly,

$$
\left|\begin{array}{ll}
\frac{a_{1} \tau}{\rho^{+}(\tau)-\tau} & k_{1}\left(\rho^{+}(\tau), \tau\right) \\
\frac{a_{1} \tau}{\rho^{-}(\tau)-\tau} & k_{1}\left(\rho^{-}(\tau), \tau\right)
\end{array}\right| \neq 0 \text { with } h_{1}\left(\rho^{ \pm}(\tau), \tau\right)=0
$$

$$
\left|\begin{array}{ll}
\frac{-a_{2} t}{r^{+}(t)-t} & k_{2}\left(r^{+}(t), t\right) \\
\frac{-a_{2} t}{r^{-}(t)-t} & k_{2}\left(r^{-}(t), t\right)
\end{array}\right| \neq 0 \text { with } h_{2}\left(r^{ \pm}(\tau), \tau\right)=0
$$

and, by using (5.7),

$$
\begin{gathered}
\left|\begin{array}{cc}
\frac{-a_{1} T}{\rho^{+}(T)-T} & k_{1}\left(\rho^{+}(T), T\right) \\
\frac{-a_{2} T}{r^{+}(T)-T} & k_{2}\left(r^{+}(T), T\right)
\end{array}\right| \neq 0 \\
\text { with } h_{1}\left(\rho^{ \pm}(T), T\right)=0, \quad h_{2}\left(r^{+}(T), T\right)=0 .
\end{gathered}
$$

Remark 5.3: From Lemma 5.1, it is seen that $T$ is independent of the value of $\pi_{1}$, and that it depends only on the sum $\frac{1}{a_{1}}+\frac{1}{a_{2}}$.

\section{Poles and Residues for the Case $a_{1} \neq a_{2}$}

In this section we determine the poles of $B(\cdot), \Phi_{0}(\cdot), \Omega_{i}(\cdot), i=1,2$, and derive equations for the residues at these poles. From these equations, it will be seen that these residues can be calculated recursively and that they all contain the factor $\phi_{0}(T)$.

With

$$
\tau_{0}^{(0)}:=T, \rho_{0}^{(0)}:=\rho^{-}\left(\tau_{0}^{(0)}\right), r_{0}^{(0)}=r^{-}\left(\tau_{0}^{(0)}\right),
$$

and $T$, as defined in Lemma 5.1, we have

(i) $\quad \frac{a_{1} \tau_{0}^{(0)}}{\rho_{0}^{(0)}-\tau_{0}} b\left(\tau_{0}^{(0)}\right)+k_{1}\left(\rho_{0}^{(0)}, \tau_{0}^{(0)}\right) \phi_{0}\left(\tau_{0}^{(0)}\right)=0$,

(ii) $\frac{-a_{2} \tau_{0}^{(0)}}{r_{0}^{(0)}-\tau_{0}} b\left(\tau_{0}^{(0)}\right)+k_{2}\left(r_{0}^{(0)}, \tau_{0}^{(0)}\right) \phi_{0}\left(\tau_{0}^{(0)}\right)=0$.

With

$$
\rho_{0}^{(1)}=\rho^{+}\left(\tau_{0}^{(0)}\right), \quad r_{1}^{(1)}:=r^{+}\left(\tau_{0}^{(0)}\right)
$$


we obtain from (4.10) and (4.11) that,

(i) $\frac{a_{1} \tau_{0}^{(0)}}{\rho_{0}^{(1)}-\tau_{0}^{(0)}} b\left(\tau_{0}^{(0)}\right)+k_{1}\left(\rho_{0}^{(1)}, \tau_{0}^{(0)}\right) \phi_{0}\left(\tau_{0}^{(0)}\right)+\omega_{2}\left(\rho_{0}^{(1)}\right)\left[\frac{d \rho+(\sigma)}{d \sigma}\right]_{\sigma=\tau_{0}^{(0)}}^{-1}=0$

(ii) $\frac{-a_{2} \tau_{0}^{(0)}}{r_{0}^{(1)}-\tau_{0}^{(0)}} b\left(\tau_{0}^{(0)}\right)+k_{2}\left(r_{1}^{(1)}, \tau_{0}^{(0)}\right) \phi_{0}\left(\tau_{0}^{(0)}\right)+\omega_{1}\left(r_{1}^{(1)}\right)\left[\frac{d r+(\sigma)}{d \sigma}\right]_{\sigma=\tau_{0}^{(0)}}^{-1}=0$.

From (2.8) and (3.4), it is readily verified that the derivative in (6.4) $(i)$ is finite and nonzero. Furthermore, the determinant, formed by the coefficients of $b\left(\tau_{0}^{(0)}\right)$ and $\phi_{0}\left(\tau_{0}^{(0)}\right)$ in (6.2) $(i)$ and (6.4) $(i)$, is nonzero; cf. (5.11). Hence, from (6.2) $(i)$ and $(6.4)(i)$ it is seen that $\omega_{2}\left(\rho_{0}^{(1)}\right)$ is nonzero and finite and proportional to $\phi_{0}\left(\tau_{0}^{(0)}\right)$, analogously for $\omega_{1}\left(r_{1}^{(1)}\right)$. Consequently,

(i) $\rho_{0}^{(1)}=\rho^{+}\left(\tau_{0}^{(0)}\right)$ is a simple pole of $\Omega_{2}(\cdot)$,

(ii) $\quad r_{1}^{(1)}=r^{+}\left(\tau_{0}^{(0)}\right)$ is a simple pole of $\Omega_{1}(\cdot)$,

(iii) $\omega_{2}\left(\rho_{0}^{(1)}\right)$ and $\omega_{1}\left(r_{1}^{(1)}\right)$ contain $\phi_{0}\left(\tau_{0}^{(0)}\right)$ as a factor.

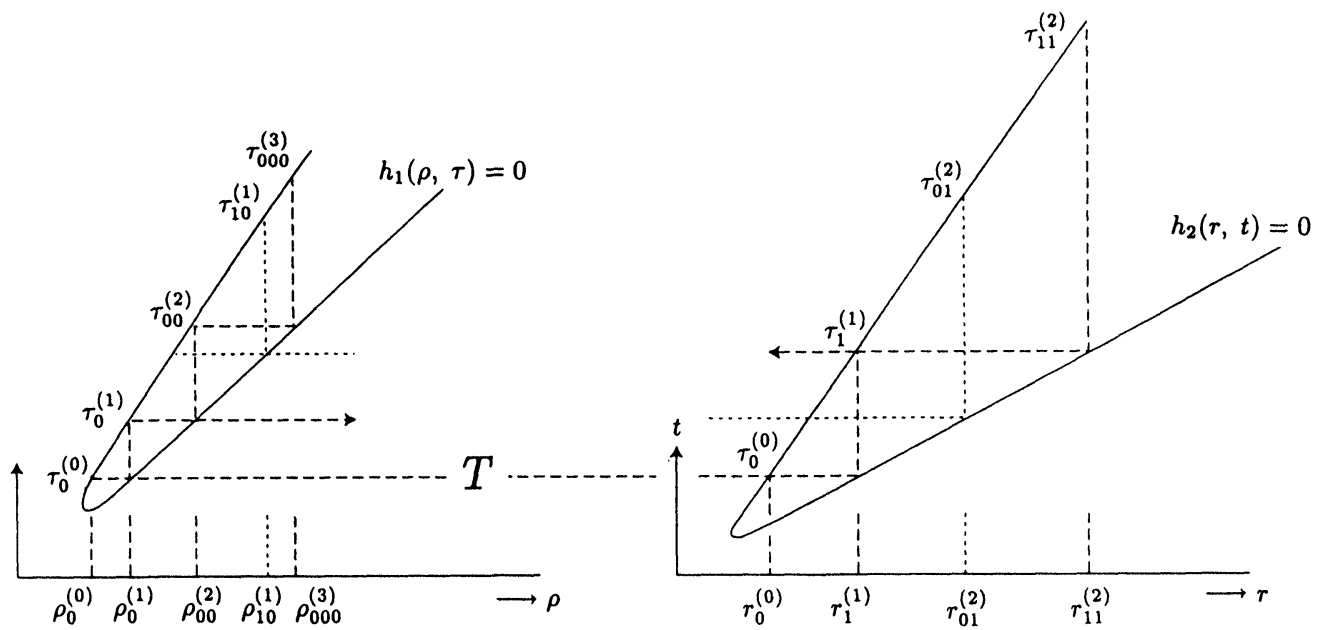

Figure 6.1

In Figure 6.1, several nodes of the tree generated by $\tau_{0}^{(0)}=T$ are shown and

$$
\rho_{00}^{(2)}=\rho^{+}\left(\tau_{0}^{(1)}\right), r_{11}^{(2)}=r^{+}\left(\tau_{1}^{(1)}\right), r_{01}^{(2)}=r^{+}\left(\tau_{0}^{(1)}\right), \rho_{10}^{(1)}=\rho^{+}\left(\tau_{1}^{(1)}\right) .
$$

Note that

$$
\begin{gathered}
\left(r^{-}\left(\tau_{0}^{(1)}\right), \tau_{0}^{(1)}\right) \text { and }\left(r_{01}^{(2)}, \tau_{0}^{(1)}\right) \text { are zero-tuples of } h_{2}(r, t)=0 \text { induced by } \tau_{0}^{(1)}, \\
\left(r^{-}\left(\tau_{0}^{(1)}\right) \text { is not shown in Figure } 6.1\right)
\end{gathered}
$$

$\left(\rho^{-}\left(\tau_{1}^{(1)}\right), \tau_{1}^{(1)}\right)$ and $\left(\rho_{10}^{(1)}, \tau_{1}^{(1)}\right)$ are zero-tuples of $h_{1}(\rho, \tau)=0$ induced by $\tau_{1}^{(1)}$, $\left(\rho^{-}\left(\tau_{1}^{(1)}\right)\right.$ is not shown in Figure 6.1).

Next, we consider (4.11) for the zero-tuple $\left(r^{-}\left(\tau_{0}^{(1)}\right), \tau_{0}^{(1)}\right)$ of $h_{2}(r, t)=0$ and (4.10) for the zero-tuple $\left(\rho^{-}\left(\tau_{1}^{(1)}\right), \tau_{1}^{(1)}\right)$ of $h_{1}(\rho, \tau)=0$, i.e., 
(i)

$$
\begin{aligned}
& \omega_{1}\left(r^{-}\left(\tau_{0}^{(1)}\right)\right)\left[\frac{d r^{-}(\sigma)}{d \sigma}\right]_{\sigma}^{-1} \tau_{0}^{(1)}+\frac{-a_{2} \tau_{0}^{(1)}}{r^{-}\left(\tau_{0}^{(1)}\right)-\tau_{0}^{(1)}} b\left(\tau_{0}^{(1)}\right) \\
& +k_{2}\left(r^{-}\left(\tau_{0}^{(1)}\right), \tau_{0}^{(1)}\right) \phi_{0}\left(\tau_{0}^{(1)}\right)=0 \\
& \omega_{2}\left(\rho^{-}\left(\tau_{1}^{(1)}\right)\right)\left[\frac{d \rho-(\sigma)}{d \sigma}\right]_{\sigma=\tau_{1}^{(1)}}^{-1}+\frac{a_{1} \tau_{1}^{(1)}}{\rho^{-}\left(\tau_{1}^{(1)}\right)-\tau_{1}^{(1)}} b\left(\tau_{1}^{(1)}\right) \\
& +k_{1}\left(\rho^{-}\left(\tau_{1}^{(1)}\right), \tau_{1}^{(1)}\right) \phi_{0}\left(\tau_{1}^{(1)}\right)=0
\end{aligned}
$$$$
\text { (ii) }
$$

Below we introduce Assumption 6.1, which implies that for the case $a_{1} \neq a_{2}$,

$$
\omega_{1}\left(r^{-}\left(\tau_{0}^{(1)}\right)\right)=0, \omega_{2}\left(\rho^{-}\left(\tau_{1}^{(1)}\right)\right)=0
$$

Hence, from (4.10) and (6.8) $(i)$, we have

$$
\begin{gathered}
\omega_{2}\left(\rho_{0}^{(1)}\right)\left[\frac{d \rho^{-}(\sigma)}{d \sigma}\right]_{\sigma=\tau_{0}^{(1)}}^{-1}+\frac{a_{1} \tau_{0}^{(1)}}{\rho_{0}^{(1)}-\tau_{0}^{(1)}} b\left(\tau_{0}^{(1)}\right)+k_{1}\left(\rho_{0}^{(1)}, \tau_{0}^{(1)}\right) \phi_{0}\left(\tau_{0}^{(1)}\right)=0 \\
-\frac{a_{2} \tau_{0}^{(1)}}{r^{-}\left(\tau_{0}^{(1)}\right)-\tau_{0}^{(1)}} b\left(\tau_{0}^{(1)}\right)+k_{2}\left(r^{-}\left(\tau_{0}^{(1)}\right), \tau_{0}^{(1)}\right) \phi_{0}\left(\tau_{0}^{(1)}\right)=0 .
\end{gathered}
$$

Here $\omega_{2}\left(\rho_{0}^{(1)}\right)$ is given by $(6.4)(i)$. The main determinant of the two equations $(6.10)$ with unknowns $b\left(\tau_{0}^{(1)}\right), \phi_{0}\left(\tau_{0}^{(1)}\right)$ is $D\left(\rho^{-}\left(\tau_{0}^{(1)}\right), r^{-}\left(\tau_{0}^{(1)}\right), \tau_{0}^{(1)}\right)$; cf. (5.3). The unique zero of determinant (5.3) in $\tau>1$ is $\tau=T=\tau_{0}^{(0)}$, cf. Lemma 5.1 , so that it is nonzero, because $\tau_{0}^{(1)}>\tau_{0}^{(0)}>1$. The derivative in $(6.10)$ is also finite and nonzero; hence, $(6.10)$ has a nonzero and finite solution $b\left(\tau_{0}^{(1)}\right), \phi_{0}\left(\tau_{0}^{(1)}\right)$.

Analogously, the equations

$$
\begin{gathered}
\omega_{1}\left(r_{1}^{(1)}\right)\left[\frac{d r^{-}(\sigma)}{d \sigma}\right]_{\sigma=r_{1}^{(1)}}^{-1}-\frac{a_{2} \tau_{1}^{(1)}}{r_{1}^{(1)}-\tau_{1}^{(1)}} b\left(\tau_{1}^{(1)}\right)+k_{2}\left(r_{1}^{(1)}, \tau_{1}^{(1)}\right) \phi_{0}\left(\tau_{1}^{(1)}\right)=0 \\
\frac{a_{1} \tau_{1}^{(1)}}{\rho^{-}\left(\tau_{1}^{(1)}\right)-\tau_{1}^{(1)}} b\left(\tau_{1}^{(1)}\right)+k_{1}\left(\rho^{-}\left(\tau_{1}^{(1)}\right), \tau_{1}^{(1)}\right) \phi_{0}\left(\tau_{1}^{(1)}\right)=0
\end{gathered}
$$

have a nonzero and finite solution $b\left(\tau_{1}^{(1)}\right), \phi_{0}^{(1)}\left(\tau_{1}^{(1)}\right)$. Consequently,

(i) $\tau_{0}^{(1)}$ and $\tau_{1}^{(1)}$ are simple poles of $B(\cdot)$ and also $\Phi_{0}(\cdot)$,

(ii) $\quad b\left(\tau_{0}^{(1)}\right), b\left(\tau_{1}^{(1)}\right), \phi_{0}\left(\tau_{0}^{(1)}\right), \phi_{0}\left(\tau_{1}^{(1)}\right)$ all contain $\phi_{0}\left(\tau_{0}^{(1)}\right)$ as a factor.

With $b\left(\tau_{0}^{(1)}\right), \phi_{0}\left(\tau_{0}^{(1)}\right)$ defined above as the solution of equations $(6.10)$ and $b\left(\tau_{1}^{(1)}\right)$, $\phi_{0}\left(\tau_{1}^{(1)}\right)$ as the solution of equations (6.11), we obtain from (4.4) and (4.11),

$$
\omega_{2}\left(\rho_{00}^{(2)}\right)\left[\frac{d \rho+(\sigma)}{d \sigma}\right]_{\sigma=\tau_{0}^{(1)}}^{-1}+\frac{a_{1} \tau_{0}^{(1)}}{\rho_{00}^{(2)}-\tau_{0}^{(1)}} b\left(\tau_{0}^{(1)}\right)+k_{1}\left(\rho_{00}^{(2)}, \tau_{0}^{(1)}\right) \phi_{0}\left(\tau_{0}^{(1)}\right)=0
$$

(ii) $\omega_{1}\left(r_{01}^{(2)}\right)\left[\frac{d r^{+}(\sigma)}{d \sigma}\right]_{\sigma=\tau_{0}^{(1)}}^{-1}+\frac{-a_{2} \tau_{0}^{(1)}}{r_{01}^{(2)}-\tau_{0}^{(1)}} b\left(\tau_{0}^{(1)}\right)+k_{2}\left(r_{01}^{(2)}, \tau_{0}^{(1)}\right) \phi_{0}\left(\tau_{0}^{(1)}\right)=0$

(i) $\quad \omega_{2}\left(\rho_{10}^{(2)}\right)\left[\frac{d \rho+(\sigma)}{d \sigma}\right]_{\sigma=\tau_{1}^{(1)}}^{-1}+\frac{a_{1} \tau_{1}^{(1)}}{\rho_{10}^{(2)}-\tau_{1}^{(1)}} b\left(\tau_{1}^{(1)}\right)+k_{1}\left(\rho_{10}^{(2)}, \tau_{1}^{(1)}\right) \phi_{0}\left(\tau_{1}^{(1)}\right)=0$ 
(ii) $\omega_{1}\left(r_{11}^{(2)}\right)\left[\frac{d r^{+}(\sigma)}{d \sigma}\right]_{\sigma=\tau_{1}^{(1)}}^{-1}+\frac{-a_{2} \tau_{1}^{(1)}}{r_{11}^{(2)}-\tau_{1}^{(1)}} b\left(\tau_{1}^{(1)}\right)+k_{2}\left(r_{11}^{(2)}, \tau_{1}^{(1)}\right) \phi_{0}\left(\tau_{1}^{(1)}\right)=0$

From (6.13) and (6.14), it is seen that

$$
\begin{aligned}
& \rho_{00}^{(2)}, \rho_{10}^{(2)} \text { and } r_{01}^{(2)}, r_{11}^{(2)} \text { are simple poles of } \Omega_{2}(\cdot) \text { and } \Omega_{1}(\cdot), \text { respectively, } \\
& \text { for which the residues follow from }(6.13) \text { and }(6.14) .
\end{aligned}
$$

Relations (6.4) and (6.8) represent the relations for the poles and residues at the zerolevel of the tree, cf. also (6.3); the relations $(6.10),(6.11),(6.13)$ and (6.14) describe the relations for the poles and residues at the first level of the tree generated by $\tau_{0}^{(0)}$. To obtain those relations at the $n$th level of the tree, we introduce, cf. (6.9), the following.

Assumption 6.1: For the case $a_{1} \neq a_{2}$, let $\tau_{\delta}^{(n)}, \delta \in\left\{0,1, \ldots, 2^{n}-1\right\}$ be an element of the tree generated by $\tau_{0}^{(0)}=T$; see Figure 3.4. Henceforth, it will be assumed, cf. Remark 2.1, that

(i) for $\delta$ even:

$$
\omega_{1}\left(r^{-}\left(\tau_{\delta}^{(n)}\right)\right)=0 \text { with } h_{2}\left(r^{-}\left(\tau_{\delta}^{(n)}\right), \tau_{\delta}^{(n)}\right)=0
$$

(ii) for $\delta$ odd:

$$
\omega_{2}\left(\rho^{-}\left(\tau_{\delta}^{(n)}\right)\right)=0 \text { with } h_{1}\left(\rho^{-}\left(\tau_{\delta}^{(n)}\right), \tau_{\delta}^{(n)}\right)=0
$$

Remark 6.1: Concerning the latter assumption, it is noted that Remark 4.2 also applies here.

Consider Figure 6.2,

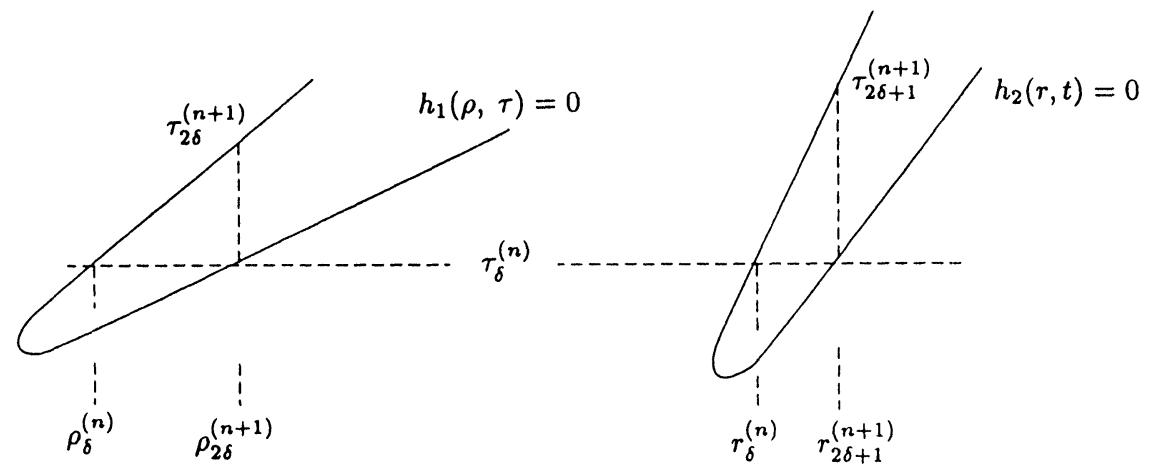

Figure 6.2

with the symbols defined by 
and

$$
\begin{gathered}
\rho_{\delta}^{(n)}:=\rho^{-}\left(\tau_{\delta}^{(n)}\right), \quad r_{\delta}^{(n)}:=r^{-}\left(\tau_{\delta}^{(n)}\right), \\
\rho_{2 \delta}^{(n+1)}:=\rho^{+}\left(\tau_{\delta}^{(n)}\right), \quad r_{2 \delta+1}^{(n+1)}=r^{+}\left(\tau_{\delta}^{(n)}\right),
\end{gathered}
$$

$$
\delta \in\left\{0, \ldots, 2^{n}-1\right\}
$$

written as a binary number, cf. (3.14).

Suppose for the present that

$$
\begin{aligned}
& \text { for } \delta \text { even: } \rho_{\delta}^{(n)} \text { is a simple pole of } \Omega_{2}(\cdot), \\
& \text { for } \delta \text { odd: } r_{\delta}^{(n)} \text { is a simple pole of } \Omega_{1}(\cdot)
\end{aligned}
$$

We consider first the case of

$$
\delta \text { being even. }
$$

Because Assumption 6.1 implies $\omega_{1}\left(r_{\delta}^{(n)}\right)=0$, for which, we have from (4.10) and (4.11),

(i) $\quad \omega_{2}\left(\rho_{\delta}^{(n)}\right)\left[\frac{d \rho^{-}(\sigma)}{d \sigma}\right]_{\sigma=\tau}^{-1}(n)+\frac{a_{1} \tau_{\delta}^{(n)}}{\rho_{\delta}^{(n)}-\tau_{\delta}^{(n)}} b\left(\tau_{\delta}^{(n)}\right)+k_{1}\left(\rho_{\delta}^{(n)}, \tau_{\delta}^{(n)}\right) \phi_{0}\left(\tau_{\delta}^{(n)}\right)=0$,

$$
-\frac{a_{2} \tau_{\delta}^{(n)}}{r_{\delta}^{(n)}-\tau_{\delta}^{(n)}} b\left(\tau_{\delta}^{(n)}\right)+k_{2}\left(r_{\delta}^{(n)}, \tau_{\delta}^{(n)}\right) \phi_{0}\left(\tau_{\delta}^{(n)}\right)=0
$$

(ii) $\quad \omega_{2}\left(\rho_{2 \delta}^{(n+1)}\right)\left[\frac{d \rho+(\sigma)}{d \sigma}\right]_{\sigma=\tau \delta}^{-1}(n)+\frac{a_{1} \tau_{\delta}^{(n)}}{\rho_{2 \delta}^{(n+1)}-\tau_{\delta}^{(n)}} b\left(\tau_{\delta}^{(n)}\right)$

$$
+k_{1}\left(\rho_{2 \delta}^{(n+1)}, \tau_{\delta}^{(n)}\right) \phi_{0}\left(\tau_{\delta}^{(n)}\right)=0
$$

(iii) $\quad \omega_{1}\left(r_{2 \delta+1}^{(n+1)}\right)\left[\frac{d r^{+}(\sigma)}{d \sigma}\right]_{\sigma=\tau_{\delta}^{(n)}}^{-1}-\frac{a_{2} \tau_{\delta}^{(n)}}{r_{2 \delta+1}^{(n+1)}-\tau_{\delta}^{(n)}} b\left(\tau_{\delta}^{(n)}\right)$

$$
+k_{2}\left(r_{2 \delta+1}^{(n+1)}, \tau_{\delta}^{(n)}\right) \phi_{0}\left(\tau_{\delta}^{(n)}\right)=0
$$

Next consider the case that

$$
\delta \text { is odd. }
$$

Then, analogously, because $\omega_{2}\left(\rho_{\delta}^{(n)}\right)=0$, we have:

$$
\begin{array}{ll}
\text { (i) } \quad \omega_{1}\left(r_{\delta}^{(n)}\right)\left[\frac{d r-(\sigma)}{d \sigma}\right]_{\sigma=\tau}^{-1}(n)+\frac{-a_{2} \tau_{\delta}^{(n)}}{r_{\delta}^{(n)}-\tau_{\delta}^{(n)}} b\left(\tau_{\delta}^{(n)}\right)+k_{2}\left(r_{\delta}^{(n)}, \tau_{\delta}^{(n)}\right) \phi_{0}\left(\tau_{\delta}^{(n)}\right)=0, \\
& \frac{a_{1} \tau_{\delta}^{(n)}}{\rho_{\delta}^{(n)}-\tau_{\delta}^{(n)}} b\left(\tau_{\delta}^{(n)}\right)+k_{1}\left(\rho_{\delta}^{(n)}, \tau_{\delta}^{(n)}\right) \phi_{0}\left(\tau_{\delta}^{(n)}\right)=0 \\
\text { (ii) } \quad & \omega_{1}\left(r_{2 \delta+1}^{(n+1)}\right)\left[\frac{d r^{+}(\sigma)}{d \sigma}\right]_{\sigma=\tau \delta^{(n)}}^{-1}+\frac{-a_{2} \tau_{\delta}^{(n)}}{r_{2 \delta+1}^{(n+1)}-\tau_{\delta}^{(n)}} b\left(\tau_{\delta}^{(n)}\right)
\end{array}
$$




$$
+k_{2}\left(r_{2 \delta+1}^{(n+1)}, \tau_{\delta}^{(n)}\right) \phi_{0}\left(\tau_{\delta}^{(n)}\right)=0
$$

(iii) $\omega_{2}\left(\rho_{2 \delta}^{(n+1)}\right)\left[\frac{d \rho+(\sigma)}{d \sigma}\right]_{\sigma=\tau}(n)+\frac{a_{1} \tau_{\delta}^{(n)}}{\rho_{2 \delta}^{(n+1)}-\tau_{\delta}^{(n)}} b\left(\tau_{\delta}^{(n)}\right)$

$$
+k_{1}\left(\rho_{2 \delta}^{(n+1)}, \tau_{\delta}^{(n)}\right) \phi_{0}\left(\tau_{\delta}^{(n)}\right)=0
$$

(6.20) $(i)$ are two equations for $b\left(\tau_{\delta}^{(n)}\right), \phi_{0}\left(\tau_{\delta}^{(n)}\right)$ and $\delta$ being even. Again, it is readily verified that the main determinant is nonzero and that the derivative in $(6.20)(i)$ is finite and nonzero; see below (6.10). From (4.6) and (6.18) it is seen that $\omega_{2}\left(\rho_{\delta}^{(n)}\right)$ is finite and nonzero. Then $(6.17)(i)$ has a nonnull solution $b\left(\tau_{\delta}^{(n)}\right)$, $\phi_{0}\left(\tau_{\delta}^{(n)}\right)$, i.e., (cf. (4.6)), for $\delta$ even:

$$
\tau_{\delta}^{(n)} \text { is a simple pole of } B(\cdot) \text { and also of } \Phi_{0}(\cdot) \text {. }
$$

From the solution of (6.20) (i), it follows from (6.22) (ii) and (iii) that, cf. (4.6), for $\delta$ even:

$$
\rho_{2 \delta}^{(n+1)} \text { and } r_{2 \delta+1}^{(n+1)} \text { are simple poles of } \Omega_{2}(\cdot) \text { and } \Omega_{1}(\cdot), \text { respectively, }
$$

and their residues are calculated from (6.20) (ii) and (6.20) (iii). Further, from (4.6) and (6.18), it follows that for $\delta$ odd:

$$
\tau_{\delta}^{(n)} \text { is a simple pole of } B(\cdot) \text { and } \Phi_{0}(\cdot),
$$

$\rho_{2 \delta}^{(n+1)}$ and $r_{2 \delta+1}^{(n+1)}$ are simple poles of $\Omega_{2}(\cdot)$ and $\Omega_{1}(\cdot)$, respectively,

and their residues are calculated from (6.22) (ii) and (6.22) (iii).

It remains to consider the hypothesis, cf. $(6.18)$, that $\omega_{2}\left(\rho_{\delta}^{(n)}\right)$ for $\delta$ even and $\omega_{1}\left(r_{\delta}^{(n)}\right)$ for $\delta$ odd are both finite and nonzero. By induction, it is seen from $(6.20)$ and from $(6.22)$ for $n=1,2, \ldots$, and $(6.10),(6.11),(6.13)$, and (6.14) that these hypotheses are indeed valid. Note that these relations show that all residues are zero or no one is zero; the first case is impossible, see the penultimate paragraph of Appendix B.

Lemma 6.1: For the case $a_{1} \neq a_{2}$ and with $T$, as defined in Lemma 5.1,

(i) every element $\tau_{\delta}^{(n)}, n=0,1,2, \ldots ; \delta \in\left\{0,1, \ldots, 2^{n}-1\right\}$, of the tree generated by $\tau_{0}^{(0)}=T$ is a simple pole of $B(\cdot)$ and also of $\Phi_{0}(\cdot)$,

(ii) $\quad \rho_{\delta}^{(n)} \equiv \rho^{-}\left(\tau_{\delta}^{(n)}\right)$ is for $\delta$ even a simple pole of $\Omega_{2}(\cdot)$,

(iii) $\quad r_{\delta}^{(n)} \equiv r^{-}\left(\tau_{\delta}^{(n)}\right)$ is for $\delta$ odd a simple pole of $\Omega_{1}(\cdot)$,

(iv) the residues $b\left(\tau_{\delta}^{(n)}\right), \phi_{0}\left(\tau_{\delta}^{(n)}\right)$ of $B(\cdot)$ and $\Phi_{0}(\cdot)$ are obtained by solving for each $\tau_{\delta}^{(n)}$, two linear equations, viz. (6.2) for $n=0,(6.10)$ and (6.11) for $n=1$, and (6.20) and (6.22) for $n=2,3, \ldots$,

$(v) \quad$ for $\delta$ even, the residues $\omega_{2}\left(\rho_{2 \delta}^{(n+1)}\right)$ and $\omega_{1}\left(r_{2 \delta+1}^{(n+1)}\right)$ are determined by $(6.8)$ for $n=0$, by (6.13) for $n=1$, by (6.20) (ii), (iii) for $n=2,3, \ldots$,

(vi) for $\delta$ odd, the residues $\omega_{2}\left(\rho_{2 \delta}^{(n+1)}\right)$ and $\omega_{1}\left(r_{2 \delta+1}^{(n+1)}\right)$ are determined by $(6.14)$ for $n=1$, and by (6.22) (ii), (iii) for $n=2,3, \ldots$, 
(vii) these residues can be calculated recursively, except for $\phi_{0}\left(\tau_{0}^{(0)}\right)$ which is a factor of every residue.

Proof: The proof follows immediately from the above analysis in this section.

Lemma 6.1 describes the equations for the residues at all nodes of the tree generated by $\tau_{0}^{(0)}=T$. But, as we have seen in Section 3 , every node $\tau_{\delta}^{(n)}$, with $\delta$ even, induces on $h_{2}(r, t)=0$ a ladder, and analogously for $\delta$ odd, a ladder on $h_{1}(\rho, \tau)=0$, see Figures 3.2 and 3.3. So we have to consider also the equations (4.9)-(4.12) for the residues at the points of the down-ladders of such induced ladders; see below (3.9).

Lemma 6.2: For the case $a_{1} \neq a_{2}$, the only poles of $B(\cdot), \Phi_{0}(\cdot), \Omega_{1}(r)$ and $\Omega_{2}(\rho)$ are those described in Lemma 6.1 .

Proof: Let $\left(\tau_{n}, \rho_{n}\right)$ be a zero-tuple of $h_{1}(\rho, \tau)$ and consider the down-ladder induced by $\tau_{n}$ on $h_{2}(r, t)=0$; see Figure 3.2 .

With

$$
\tau_{n}=\tilde{t}_{n}, \widetilde{r}_{n}=r^{-}\left(\tilde{t}_{n}\right), \tilde{t}_{n-1}=t^{-}\left(\tilde{r}_{n}\right), \tilde{r}_{n-1}=r^{-}\left(\tilde{t}_{n-1}\right), \ldots
$$

it follows from (4.10) that

$$
\begin{aligned}
& -\omega_{1}\left(\tilde{r}_{n}\right)=\left[\frac{-a_{2} \tilde{t}_{n}}{\tilde{r}_{n}-\tilde{t}_{n}} b\left(\tilde{t}_{n}\right)+k_{2}\left(\tilde{r}_{n}, \tilde{t}_{n}\right) \phi_{0}\left(\tilde{t}_{n}\right)\right]\left[\frac{d t+(\sigma)}{d \sigma}\right]_{\sigma=\tilde{r}_{n}}^{-1} \\
= & {\left[\frac{-a_{2} \tilde{t}_{n-1}}{\tilde{r}_{n}-\tilde{t}_{n-1}}\right] b\left(\tilde{t}_{n-1}\right)+k_{2}\left(\tilde{r}_{n}, \tilde{t}_{n-1}\right) \phi_{0}\left(\tilde{t}_{n-1}\right)\left[\frac{d t-(\sigma)}{d \sigma}\right]_{\sigma}=\tilde{r}_{n} }
\end{aligned}
$$

and

$$
\begin{gathered}
-\omega_{1}\left(\tilde{r}_{i}\right)=\left[\frac{-a_{2} \tilde{t}_{i}}{\tilde{r}_{i}-\tilde{t}_{i}} b\left(\tilde{t}_{i}\right)+k_{2}\left(\tilde{r}_{i}, \tilde{t}_{i}\right) \phi_{0}\left(\tilde{t}_{i}\right)\right]\left[\frac{d t+(\sigma)}{d \sigma}\right]_{\sigma=\tilde{r}_{i}}^{-1} \\
=\frac{-a_{2} \tilde{t}_{i-1}}{\tilde{r}_{i}-\tilde{t}_{i-1} b\left(\tilde{t}_{i-1}\right)+k_{2}\left(\tilde{r}_{i}, \tilde{t}_{i-1}\right) \phi_{0}\left(\tilde{t}_{i-1}\right)}\left[\frac{d t-(\sigma)}{d \sigma}\right]_{\sigma=\tilde{r}_{i-1}}^{-1}
\end{gathered}
$$

for $i=n-1, n-2, \ldots, \nu$, with $\nu$ being the index at which the down-ladder is stopped (cf. below (3.9)), i.e., the index, for which

so that; cf. (4.8),

$$
0<\tilde{t}_{\nu}<1 \text { or } 0<\tilde{r}_{\nu}<1
$$

$$
b\left(\tau_{\nu}\right)=0, \phi_{0}\left(\tau_{\nu}\right)=0 \text { or }-\omega_{1}\left(\widetilde{r}_{\nu}\right)=0
$$

and, cf. Assumption 6.1,

$$
\omega_{1}\left(\widetilde{r}_{n}\right)=0
$$

The set of relations (6.27), (6.28) and (6.29) is insufficient to determine the unknown residues $\omega_{1}\left(\tilde{r}_{i}\right), b\left(\tilde{t}_{i}\right)$ and $\phi_{0}\left(\tilde{t}_{i}\right)$. However, Assumption 4.1 leads to the conclusion that the only solution of this set of relations is the zero-solution, i.e.,

at all induced down-ladders, the residues at the elements of these down-ladders are zero, so that these elements cannot be poles of $B(\cdot), \Phi_{0}(\cdot), \Omega_{1}(\cdot)$ and $\Omega_{2}(\cdot)$. 
To see this, first note that every element of the tree generated by $\tau_{0}^{(0)}=T$ induces down-ladders on $h_{1}(\rho, \tau)=0$ or on $h_{2}(r, t)=0$, and elements of down-ladders again induce down-ladders. Since the tree generated by $\tau_{0}^{(0)}$ consists of an infinite but countable number of nodes, it follows that the finite part of $h_{1}(\rho, \tau)=0$ with $\rho \in$ $\left[\rho^{-}(1), \rho^{+}(1)\right]$ contains an infinitely countable set of elements $(\rho, \tau)$ stemming from the induced down-ladders, similarly for $h_{2}(r, t)=0$. Hence, Assumption 4.1 requires that the residues at these elements are all zero, because of a meromorphic function can have at most a finite number of poles in a finite domain. It is readily seen (cf. (6.27), (6.28)) from Assumption 6.1 together with the relations (4.8)-(4.11), that all residues at points of the down-ladders induced by any point of the tree are indeed zero. Consequently, for the various assumptions so far introduced, cf. Assumptions $4.1,4.2,5.1$ and 6.1 , the only poles of $B(\cdot), \Phi_{0}(\cdot), \Omega_{i}(\cdot), i=1,2$, are those mentioned in Lemma 6.1 .

\section{Asymptotic Behavior of the Residue, $a_{1} \neq a_{2}$}

For further analysis of the functions $B(\cdot), \Phi_{0}(\cdot), \Omega_{1}(\cdot)$ and $\Omega_{2}(\cdot)$, we require the asymptotic behavior for $n \rightarrow \infty$ of the residues of these functions at their poles; see the preceding section.

First we consider the ladder generated by $\tau_{0}^{(0)}=T$ on $h_{1}(\rho, \tau)=0$, i.e., the set of nodes $\tau_{\delta_{j}^{(n)}}^{(n)}, \delta_{j}^{(n)}=0, n=0,1,2, \ldots$, which is the extreme left branch of the tree generated by $\left(T, \rho^{-}(T)\right)$.

Put, cf. Figure 3.2, for $n=0,1,2, \ldots$,

$$
\begin{gathered}
\tau_{n}:=\tau_{0}^{(n)}, \tau_{0}^{(0)}=T \\
\rho_{n}:=\rho^{-}\left(\tau_{n}\right), \rho_{n+1}=\rho^{+}\left(\tau_{n}\right)=\rho^{-}\left(\tau_{n+1}\right), h_{1}\left(\rho_{n}, \tau_{n}\right)=0 \\
\tau_{n}=\tau^{+}\left(\rho_{n}\right), \tau_{n+1}=\tau^{+}\left(\rho_{n+1}\right), \quad h_{1}\left(\rho_{n+1}, \tau_{n+1}\right)=0 .
\end{gathered}
$$

From (4.8), we obtain

$$
\begin{gathered}
\omega_{2}\left(\rho_{n+1}\right)+\left[\frac{a_{1} \tau_{n+1}}{\rho_{n+1}-\tau_{n+1}} b\left(\tau_{n+1}\right)+k_{1}\left(\rho_{n+1}, \tau_{n+1}\right) \phi_{0}\left(\tau_{n+1}\right)\right] \\
\times\left[\frac{d \tau^{+}(\sigma)}{d \sigma}\right]_{\sigma=\rho_{n+1}}^{-1}=0, \\
\omega_{2}\left(\rho_{n+1}\right)+\left[\frac{a_{1} \tau_{n}}{\rho_{n+1}-\tau_{n}} b\left(\tau_{n}\right)+k_{1}\left(\rho_{n+1}, \tau_{n}\right) \phi_{0}\left(\tau_{n}\right)\right] \\
\times\left[\frac{d \tau^{-}(\sigma)}{d \sigma}\right]_{\sigma}^{-1}=\rho_{n+1}=0 .
\end{gathered}
$$

Elimination of $\omega_{2}\left(\rho_{n+1}\right)$ yields

$$
\left[\frac{a_{1} \tau_{n+1}}{\rho_{n+1}-\tau_{n+1}} b\left(\tau_{n+1}\right)+\frac{k_{1}\left(\rho_{n+1}, \tau_{n+1}\right)}{\tau_{n+1}} \tau_{n+1} \phi_{0}\left(\tau_{n+1}\right)\right]\left[\frac{d \tau^{+}(\sigma)}{d \sigma}\right]_{\sigma=\rho_{n+1}}^{-1}
$$




$$
=\left[\frac{a_{1} \tau_{n}}{\rho_{n+1}-\tau_{n}} b\left(\tau_{n}\right)+\frac{k_{1}\left(\rho_{n+1}, \tau_{n}\right)}{\tau_{n}} \tau_{n} \phi_{0}\left(\tau_{n}\right)\right]\left[\frac{d \tau^{-}(\sigma)}{d \sigma}\right]_{\sigma=\rho_{n+1}}^{-1} .
$$

Further, with

from $(6.15)$,

$$
\tilde{r}_{n+1}=r^{-1}\left(\tau_{n+1}\right), h_{2}\left(\tilde{r}_{n+1}, \tau_{n+1}\right)=0
$$

$$
\frac{-a_{2} \tau_{n+1}}{\widetilde{r}_{n+1}-\tau_{n+1}} b\left(\tau_{n+1}\right)+\frac{k_{2}\left(\tilde{r}_{n+1}, \tau_{n+1}\right)}{\tau_{n+1}} \tau_{n+1} \phi_{0}\left(\tau_{n+1}\right)=0 .
$$

For $n \rightarrow \infty$ we have, cf. (2.8), (3.16)-(3.19), since $\tau_{n} \rightarrow \infty$ implies $\rho_{n} \rightarrow \infty$ and $\tilde{r}_{n} \rightarrow \infty$ :

$$
\begin{gathered}
\frac{a_{1} \tau_{n+1}}{\rho_{n+1}-\tau_{n+1}} \rightarrow \frac{a_{1}}{R_{1-1}^{-}}, \quad \frac{k_{1}\left(\rho_{n+1}, \tau_{n+1}\right)}{\tau_{n+1}} \rightarrow a_{2} \pi_{2}\left(a_{1}-R_{1}^{-}\right), \\
\frac{a_{1} \tau_{n}}{\rho_{n+1}-\tau_{n}} \rightarrow \frac{a_{1}}{R_{1}^{+}-1}, \quad \frac{k_{1}\left(\rho_{n+1}, \tau_{n}\right)}{\tau_{n}} \rightarrow a_{2} \pi_{2}\left(a_{1}-R_{1}^{+}\right), \\
\frac{a_{2} \tau_{n+1}}{\widetilde{r}_{n+1}-\tau_{n+1}} \rightarrow \frac{a_{2}}{R_{2}^{-}-1}, \frac{k_{2}\left(\widetilde{r}_{n+1}, \tau_{n+1}\right)}{\tau_{n+1}} \rightarrow a_{1} \pi_{1}\left(a_{2}-R_{2}^{-}\right), \\
\left.\frac{d \tau^{ \pm}(\sigma)}{d \sigma}\right|_{\sigma}=\rho_{n+1} \rightarrow \frac{b T_{1}^{ \pm}-2 a_{2}}{2 a_{1} a_{2} T_{1}^{ \pm}-b}, \\
\frac{\tau_{n+1}}{\tau_{n}}=\frac{\tau^{+}\left(\rho+\left(\tau_{n}\right)\right)}{\rho^{+}\left(\tau_{n}\right)} \frac{\rho^{+}\left(\tau_{n}\right)}{\tau_{n}} \rightarrow \frac{R_{1}^{+}}{R_{1}^{-}}=\frac{T_{1}^{+}}{T_{1}^{-}}=R_{1}^{+} T_{1}^{+}>1 .
\end{gathered}
$$

Lemma 7.1: For the elements $\left(\rho_{n}, \tau_{n}\right)$ of the extreme left ladder of the tree generated by $\left(T, \rho_{0}(T)\right)$, cf. (7.1), holds: for $n=0,1,2, \ldots$,

(i) $\lim _{m \rightarrow \infty} \frac{b\left(\tau_{m+n}\right)}{b\left(\tau_{m}\right)}=\mu_{1}^{n}$

(ii) $\lim _{m \rightarrow \infty} \frac{\tau_{m+n} \phi_{0}\left(\tau_{m+n}\right)}{\tau_{m} \phi_{0}\left(\tau_{m}\right)}=\mu_{1}^{n}, \quad \lim _{m \rightarrow \infty} \frac{\phi_{0}\left(\tau_{m+1}\right)}{\phi_{0}\left(\tau_{m}\right)}=\mu_{1} \lambda_{1}$,

(ii) $\lim _{m \rightarrow \infty} \frac{\omega_{2}\left(\rho_{m+n}\right)}{\omega_{2}\left(\rho_{m}\right)}=\mu_{1}^{n}, \quad \lim _{m \rightarrow \infty} \frac{\phi_{0}\left(\tau_{m+n}\right)}{\phi_{0}\left(\tau_{m}\right)}=\left(\mu_{1} \lambda_{1}\right)^{n}$

with

(iv) $0<\lambda_{1}$ : $=\frac{R_{1}^{-}}{R_{1}^{+}}<1, \quad \mu_{1}=\lambda_{1}^{-1} \frac{R_{1}^{-}-1}{R_{1}^{+}-1} \frac{\pi_{1} R_{2}^{-}+\pi_{2} R_{1}^{+}}{\pi_{1} R_{2}^{-}+\pi_{2} R_{1}^{-}}<0$,

(v) $\lim _{n \rightarrow \infty} \frac{b\left(\tau_{n}\right)}{\tau_{n} \phi_{0}\left(\tau_{n}\right)}=-\pi_{1} R_{2}^{-}$,

(vi) $\lim _{n \rightarrow \infty} \frac{\omega_{2}\left(\rho_{n}\right)}{\tau_{n} \phi_{0}\left(\tau_{n}\right)}=\frac{a_{1} R_{1}^{-}}{\left(R_{1}^{-}-1\right)}\left(\pi_{1} R_{2}^{-}+\pi_{2} R_{1}^{-}\right)<0$;

for the elements $\left(r_{n}, t_{n}\right)$ of the right-most ladder of the tree generated by $\left(T, r^{-}(T)\right)$ holds; for $n=0,1,2, \ldots$, 
(i) $\lim _{m \rightarrow \infty} \frac{b\left(t_{m+n}\right)}{b\left(t_{m}\right)}=\mu_{2}^{n}$

(ii) $\lim _{m \rightarrow \infty} \frac{t_{m+n} \phi_{0}\left(t_{m+n}\right)}{t_{m} \phi_{0}\left(t_{m}\right)}=\mu_{2}^{n}, \quad \lim _{m \rightarrow \infty} \frac{\phi_{0}\left(t_{m+1}\right)}{\phi_{0}\left(t_{m}\right)}=\mu_{2} \lambda_{2}$

(iii) $\lim _{m \rightarrow \infty} \frac{\omega_{1}\left(r_{m+n}\right)}{\omega_{1}\left(r_{m}\right)}=\mu_{2}^{n}, \lim _{m \rightarrow \infty} \frac{\phi_{0}\left(t_{m+n}\right)}{\phi_{0}\left(t_{m}\right)}=\left(\mu_{2} \lambda_{2}\right)^{n}$

with

(iv) $0<\lambda_{2}:=\frac{R_{2}^{-}}{R_{2}^{+}}<1, \quad \mu_{2}=\lambda_{2}^{-1} \frac{R_{2}^{-}-1}{R_{2}^{+}-1} \frac{\pi_{2} R_{1}^{-}+\pi_{1} R_{2}^{+}}{\pi_{2} R_{1}^{-}+\pi_{1} R_{2}^{-}}<0$,

(v) $\lim _{n \rightarrow \infty} \frac{b\left(t_{n}\right)}{t_{n} \phi_{0}\left(t_{n}\right)}=-\pi_{2} R_{1}^{-}$,

(vi) $\lim _{n \rightarrow \infty} \frac{\omega_{1}\left(r_{n}\right)}{\tau_{n} \phi_{0}\left(\tau_{n}\right)}=\frac{a_{2} R_{2}^{-}}{R_{2}^{-}-1}\left(\pi_{2} R_{1}^{-}+\pi_{1} R_{2}^{-}\right)<0$.

Proof: From (7.5) and (7.6), it follows that the following limit exists and is given by, cf. (3.20),

$$
\lim _{n \rightarrow \infty} \frac{b\left(\tau_{n+1}\right)}{\tau_{n+1} \phi_{0}\left(\tau_{n+1}\right)}=\frac{a_{1}}{a_{2}} \pi_{1}\left(R_{2}^{-}-1\right)\left(a_{2}-R_{2}^{-}\right)=-\pi_{1} R_{2}^{-},
$$

so that $(7.7)(v)$ has been proved.

From (7.4), (7.6) and (7.7) $(v)$, it is seen that the first limit in (7.7) (ii) exists for $n=1$ and from this result, the relation for the second limit in (7.7) (ii) follows by using the definition of $\lambda_{1}$, cf. (7.7) $(i v)$, and the last relation of (7.6). The second relation in (7.7) (iii) follows immediately from the first one in (7.7) (ii).

Next, we let $n \rightarrow \infty$ in (7.4). We then obtain by using (3.16), (3.18), (7.1) and $(7.7)(v)$

$$
\begin{aligned}
& {\left[\frac{a_{1}}{R_{1}^{-}-1}\left(-\pi_{1} R_{2}^{-}\right)+a_{2} \pi_{2}\left(a_{1}-R_{1}^{-}\right)\right] R_{1}^{-} \mu_{1}} \\
& =\left[\frac{a_{1}}{R_{1}^{+}-1}\left(-\pi_{1} R_{2}^{-}\right)+a_{2} \pi_{2}\left(a_{1}-R_{1}^{+}\right)\right] R_{1}^{+} .
\end{aligned}
$$

By using (3.20), the second relation in (7.7) $(i v)$ is readily obtained, since $0<R_{1}^{-}<$ $1, R_{1}^{+}>1$. The inequality on the left of the first relation of (7.7) (iv) follows from $R_{1}^{+}>R_{1}^{-}>0$.

From (7.7) $(i i),(v)$ and from

$$
\begin{aligned}
-\pi_{1} R_{2}^{-} & =\lim _{n \rightarrow \infty} \frac{b\left(\tau_{n+1}\right)}{b\left(\tau_{n}\right)} \frac{b\left(\tau_{n}\right)}{\tau_{n} \phi_{0}\left(\tau_{n}\right)} \frac{\tau_{n} \phi_{0}\left(\tau_{n}\right)}{\tau_{n+1} \phi_{0}\left(\tau_{n+1}\right)} \\
& =-\pi_{1} R_{2}^{-} \frac{1}{\mu_{1}} \lim _{n \rightarrow \infty} \frac{b\left(\tau_{n+1}\right)}{b\left(\tau_{n}\right)}
\end{aligned}
$$

the relation $(7.7)(i)$ readily follows.

From (7.2), we obtain for $n \rightarrow \infty$,

$$
\lim _{n \rightarrow \infty} \frac{\omega_{2}\left(\rho_{n+1}\right)}{\tau_{n+1} \phi_{0}\left(\tau_{n+1}\right)}+\frac{a_{1}}{R_{1}^{-}-1}\left(-\pi_{1} R_{2}^{-}\right)+a_{2} \pi_{2}\left(a_{1}-R_{1}^{-}\right) R_{1}^{-}=0 .
$$


So, by using (3.20), we obtain (7.7) (vi). From

$$
\frac{\omega_{2}\left(\rho_{n+1}\right)}{\tau_{n+1} \phi_{0}\left(\tau_{n+1}\right)}=\frac{\omega_{2}\left(\rho_{n+1}\right)}{\omega_{2}\left(\rho_{n}\right)} \frac{\omega_{2}\left(\rho_{n}\right)}{\tau_{n} \phi_{0}\left(\tau_{n}\right)} \frac{\tau_{n} \phi_{0}\left(\tau_{n}\right)}{\tau_{n+1} \phi_{0}\left(\tau_{n+1}\right)},
$$

it follows, because the first and third quotient have the same limit, cf. (7.7), whereas that of the last quotient is equal to $\mu_{1}^{-}$, that

$$
\lim _{n \rightarrow \infty} \frac{\omega_{2}\left(\rho_{n+1}\right)}{\omega_{2}\left(\rho_{n}\right)}=\mu_{1}
$$

Consequently, the first relation in (7.7) (iii) follows. Hence, (7.7) is proved, and (7.8) follows by symmetry.

The lemma above describes only the asymptotic behavior of the various residues at those nodes of the tree generated by $\left(T, \rho^{-}(T)\right)$, which belong to the left- or rightmost branch of that tree, i.e., at the nodes

$$
\tau_{0}^{(m+n)} \text { and } \tau_{\delta}^{(n)} \text { with } \delta=2^{n}-1
$$

To consider the asymptotics for $n \rightarrow \infty$ of the residues at a generic point

we write

$$
\tau_{\delta}^{(m+n)}, \delta \in\left\{0,1, \ldots, 2^{m+n}-1\right\}
$$

$$
\begin{gathered}
\delta=e_{m} 2^{n}+d_{n} \\
e_{m} \in\left\{0, \ldots, 2^{m}-1\right\}, d_{n} \in\left\{0,1, \ldots, 2^{n}-1\right\} .
\end{gathered}
$$

Hence, $\tau_{\delta}^{(m+n)}, \quad n=0,1,2, \ldots$, is the tree generated by $\tau_{e_{m}}^{(m)}$, and it is a subtree of that generated by $T$. Now, write $d_{n}$ as a binary number and in this binary representation denote by

$$
\begin{aligned}
& d_{1}^{(n)} \text { the number of zeros, } \\
& d_{2}^{(n)} \text { the number of ones, }
\end{aligned}
$$

so that

$$
d_{1}^{(n)}+d_{2}^{(n)}=n
$$

It readily follows from (7.6), (7.9) and (7.10) that for every finite $n$ : for $m \rightarrow \infty$,

$$
\frac{\tau_{\delta}^{(m+n)}}{\tau_{e_{m}}^{(m)}} \rightarrow\left\{T_{1}^{+} R_{1}^{+}\right\}^{d_{1}^{(n)}}\left\{T_{2}^{+} R_{2}^{+}\right\}^{d_{2}^{(n)}}=\lambda_{1}^{-d_{1}^{(n)}} \lambda_{2}^{-d_{2}^{(n)}}>1
$$

Lemma 7.2: Let $\tau_{\delta}^{(m+n)}, m=0,1,2, \ldots ; n=0,1,2, \ldots$, be a generic element of the tree generated by $\left(T, \rho^{-}(T)\right)$, then: for $n=0,1,2, \ldots$,

(i) $\lim _{m \rightarrow \infty} \frac{b\left(\tau_{\delta}^{(m+n)}\right)}{b\left(\tau_{e_{m}^{(m)}}^{(m)}\right.}=\mu_{1}^{d_{1}^{(n)}} \mu_{2}^{d_{2}^{(n)}}$,

(ii) $\lim _{m \rightarrow \infty} \frac{\tau_{\delta}^{(m+n)} \phi_{0}\left(\tau_{\delta}^{(m+m)}\right)}{\tau_{e_{m}}^{(m)} \phi_{0}\left(\tau_{e_{m}}^{(m)}\right)}=\mu_{1}^{d_{1}^{(n)} \mu_{2}{ }_{2}^{(n)}}$ 
(iii) $\lim _{m \rightarrow \infty} \frac{\omega_{i}\left(\rho_{\delta}^{(m+n)}\right)}{\omega_{i}\left(\rho_{e_{m}}^{(m+n)}\right)}=\mu_{1}^{d_{1}^{(n)}} \mu_{2}^{d_{2}^{(n)}}, i=1,2$

with $e_{m}, d_{n}, d_{1}^{(n)}, d_{2}^{(n)}$, for given $\delta \in\left\{0,1, \ldots, 2^{m+n}-1\right\}$, as defined in (7.9) and (7.10), and, cf. (6.17),

$$
\begin{gathered}
\rho_{\delta}^{(m+n)}=\rho^{-}\left(\tau_{\delta}^{(m+n)}\right) \text { for } \delta \text { even } \\
=r^{-}\left(\tau_{\delta}^{(m+n)}\right) \text { for } \delta \text { odd } .
\end{gathered}
$$

Proof: For $\delta=e_{m} 2^{n}+d$ consider, the tree generated by $\tau_{e_{m}}^{(m)}$, which is a subtree of the tree generated by $\tau_{0}^{(0)}=T$. Apply for this subtree Lemma 7.1 with $n=1$. Next, apply again this lemma with $n=1$ for the elements

$$
\tau_{\delta}^{(m+1)} \text { with } \delta=2 e_{m}+d_{1}, d_{1} \in\{0,1\}
$$

then, (7.13) $(i)$, for $n=1$, follows from (7.7) $(i)$ and (7.8) (i). Apply Lemma 7.1 again with $n=1$ for the subtree generated by $\tau_{\delta}^{(m+1)}$ as given in (7.14), then (7.13) (i) follows for

$$
\tau_{\delta}^{(m+2)} \text { with } \delta=2^{2} e_{m}+d_{2}, d_{2} \in\{0,1,2,3\}
$$

Repeating this procedure leads to (7.13) (i). The statements (7.13) (ii) and (iii) are similarly proved.

\section{The Solution for the Case $a_{1} \neq a_{2}$}

We introduce the following meromorphic functions.

For $\tau_{\delta}^{(n)}, \delta \in\left(0,1,2, \ldots, 2^{n}-1\right), n=0,1,2, \ldots$, a node of the $n$th level of the tree generated by $\tau_{0}^{(0)}=T$, cf. Section 3 , and with

$$
\begin{aligned}
& \rho_{\delta}^{(n)}=\rho^{-}\left(\tau_{\delta}^{(n)}\right), h_{1}\left(\rho^{-}\left(\tau_{\delta}^{(n)}\right), \tau_{\delta}^{(n)}\right)=0, \delta \text { even, } \\
& r_{\delta}^{(n)}=r^{-}\left(\tau_{\delta}^{(n)}\right), h_{2}\left(r^{-}\left(\tau_{\delta}^{(n)}\right), \tau_{\delta}^{(n)}\right)=0, \delta \text { odd },
\end{aligned}
$$

set for nonnegative integers $m_{b}, m_{\phi}, m_{2}, m_{1}$ :

(i) $\widetilde{B}(\tau):=\sum_{n=0}^{\infty} \sum_{\delta \in \mathscr{B}_{n}} \frac{b_{\delta}^{(n)}}{\tau-\tau_{\delta}^{(n)}}\left(\frac{\tau}{\tau_{\delta}^{(n)}}\right)^{m b}$,

(ii) $\quad \widetilde{\Phi}_{0}(\tau):=\sum_{n=0}^{\infty} \sum_{\delta \in \mathscr{B}_{n}} \frac{\phi_{\delta}^{(n)}}{\tau-\tau_{\delta}^{(n)}}\left(\frac{\tau}{\tau_{\delta}^{(n)}}\right)^{m_{\phi}}$,

(iii) $\quad \tilde{\Omega}_{2}(\rho):=\sum_{n=1}^{\infty} \sum_{\delta \in \mathscr{B}_{n}} \frac{\omega_{2 \delta}^{(n)}}{\rho-\rho_{\delta}^{(n)}}\left(\frac{\rho}{\rho_{\delta}^{(n)}}\right)^{m_{2}}$,

(iv) $\widetilde{\Omega}_{1}(r):=\sum_{n=1}^{\infty} \sum_{\delta \in \mathscr{B}_{n}} \frac{\omega_{1 \delta}^{(n)}}{r-r_{\delta}^{(n)}}\left(\frac{r}{r_{\delta}^{(n)}}\right)^{m_{1}}$,

$$
\mathscr{B}_{n}=\left\{0,1,2, \ldots, 2^{n}-1\right\}
$$


and where, cf. (4.6),

$$
\left.b_{\delta}^{(n)} \equiv b\left(\tau_{\delta}^{(n)}\right), \phi_{\delta}^{(n)} \equiv \phi_{0}\left(\tau_{\delta}^{(n)}\right), \omega_{2 \delta}^{(n)} \equiv \omega_{2}\left(\rho_{\delta}^{(n)}\right), \omega_{1 \delta}^{(n)}\right) \equiv \omega_{1}\left(r_{\delta}^{(n)}\right) .
$$

First, we have to determine the values of $m_{\ldots}$ for which the right-hand sides of $(8.2)$ are well-defined meromorphic functions; cf. [15].

Consider first the function in (8.2) (i). From (7.12) it follows that $\widetilde{B}(\tau)$, as defined in (8.2) $(i)$, has only a finite number of poles in any finite interval. From Lemma 7.2 and (7.12), we have with

$$
\delta=e_{k} 2^{n}+d_{n}, d_{1}^{(n)}, d_{2}^{(n)}, \text { as defined in }(7.10),
$$

for $k \rightarrow \infty$,

$$
\begin{gathered}
\frac{b_{\delta}^{(k+n)}}{\left[\tau_{\delta}^{(k+n)}\right]^{m+1}} / \frac{b_{e_{k}}^{(k)}}{\left[\tau_{e_{k}}^{(k)}\right]^{m+1}} \rightarrow \frac{\mu_{1}^{d_{1}^{(n)}} \mu_{2}^{(n)}}{\left[\lambda_{1}^{-d_{1}^{(n)}} \lambda_{2}^{-d_{2}^{(n)}}\right]^{m+1}} \\
=\left[\mu_{1} \lambda_{1}^{m+1}\right]^{d_{1}^{(n)}}\left[\mu_{2} \lambda_{2}^{m+1}\right]^{d_{2}^{(n)}} .
\end{gathered}
$$

Because $0<\lambda_{i}<1, i=1,2$, and $d_{1}^{(n)}+d_{2}^{(n)}=n$, we have

$$
\sum_{d_{1}^{(n)}=0}^{n}\left(\begin{array}{c}
n \\
d_{1}^{(n)}
\end{array}\right)\left[\mu_{1} \lambda_{1}^{m+1}\right]^{d_{1}^{(n)}}\left[\mu_{2} \lambda_{2}^{m+1}\right]^{d_{2}^{(n)}}=\left[\mu_{1} \lambda_{1}^{m+1}+\mu_{2} \lambda_{2}^{m+1}\right]^{n} .
$$

Obviously, a unique, finite nonnegative number $\tilde{M}$ may be defined by

(i) $\quad\left|\mu_{1} \lambda_{1}^{\tilde{M}+1}+\mu_{2} \lambda_{2}^{\tilde{M}+1}\right|<1<\left|\mu_{1} \lambda_{1}^{\tilde{M}}+\mu_{2} \lambda_{2}^{\tilde{M}}\right|$ if $\left|\mu_{1} \lambda_{1}+\mu_{2} \lambda_{2}\right| \geq 1$;

(ii) $\quad \tilde{M}=0$ if $\left|\mu_{1} \lambda_{1}+\mu_{2} \lambda_{2}\right|<1$;

note that $\mu_{1}$ and $\mu_{2}$ are both negative; cf. (7.7) (iv), (7.8) (iv).

Hence, for $m_{b} \geq M$, it is seen that the right-hand side of (8.2) (i) converges absolutely for every $\tau$ with $|\tau|<R$, for every finite $R$, whenever terms with poles $\tau_{\delta}^{(n)},\left|\tau_{\delta}^{(n)}\right|<R$, are deleted from this sum. Consequently, the sum in the righthand side of $(8.2)(i)$ is a well-defined meromorphic function for $m_{b} \geq \widetilde{M}_{\dot{\sim}}$ The same conclusion is reached for the sums in (8.2) (iii) and (8.2) (iv), i.e., $m_{2} \geq \dot{\tilde{M}}, m_{1} \geq \tilde{M}$. Next, consider (8.2) (ii). A calculation analogous to that in (8.5) yields, by using (7.7) (ii), that the meromorphic function in (8.2) (ii) is well-defined for $m_{\phi} \geq$ $\max (0, \tilde{M}-1)$.

Lemma 8.1: For $a_{1} \neq a_{2}, \frac{1}{a_{1}}+\frac{1}{a_{2}}>1$, the functions $\widetilde{B}(\cdot), \widetilde{\Phi}(\cdot), \widetilde{\Omega}_{i}, i=1,2$, are well-defined meromorphic functions for

$$
m_{b} \geq \tilde{M}, m_{2} \geq \tilde{M}, m_{1} \geq \tilde{M}, m_{\phi} \geq \max (0, \tilde{M}-1)
$$

with $\tilde{M}$ as defined in $(8.6) ; \widetilde{B}(\tau)$ and $\widetilde{\Phi}_{0}(\tau)$ are both regular for $|\tau|<T=$ $\left(\frac{1}{a_{1}}+\frac{1}{a_{2}}\right)^{2}, \widetilde{\Omega}_{2}(\rho)$ is regular for $|\rho|<\rho+\left(\tau_{0}^{(0)}\right)=a_{1}\left(\frac{1}{a_{1}}+\frac{1}{a_{2}}\right)^{3}+\frac{a_{1}}{a_{2}}\left(\frac{1}{a_{1}}+\frac{1}{a_{2}}\right)$, and $\widetilde{\Omega}_{1}(r)$ for $|r|<a_{2}\left(\frac{1}{a_{1}}+\frac{1}{a_{2}}\right)^{3}+\frac{a_{2}}{a_{1}}\left(\frac{1}{a_{2}}+\frac{1}{a_{2}}\right)$.

Proof: The first statement has been proved above, the other statements follow 
from Lemma 6.2 and $\tau_{0}^{(0)}=T>1, \tau_{\delta}^{(n)}>T$,

$$
\rho_{\delta}^{(n)} \geq \rho^{+}\left(\tau_{0}^{(0)}\right)=a_{1}\left(\frac{1}{a_{1}}+\frac{1}{a_{2}}\right)^{3}+\frac{a_{1}}{a_{2}}+\left(\frac{1}{a_{1}}+\frac{1}{a_{2}}\right), \delta \in \mathfrak{B}_{n}, n=1,2, \ldots
$$

and, analogously, for $\widetilde{\Omega}_{1}(r)$.

Next, we introduce four polynomials, viz. $\widehat{B}(\cdot), \widehat{\Phi}_{0}(\cdot), \widehat{\Omega}_{1}(\cdot)$, with degrees $\widehat{N}_{b}$, $\widehat{N}_{\phi}, \widehat{N}_{i}, i=1,2$, and put

$$
\begin{gathered}
B(\tau)=\widehat{B}(\tau)+\widetilde{B}(\tau) \text { with } \widehat{B}(\tau)=\sum_{k=0}^{\widehat{N}_{b}} \widehat{B}_{k} \tau^{k}, \\
\Phi_{0}(\tau)=\widehat{\Phi}_{0}(\tau)+\widetilde{\Phi}_{0}(\tau) \text { with } \widehat{\Phi}_{0}(\tau)=\sum_{k=0}^{\widehat{N}_{\phi}} \widehat{\Phi}_{0 k} \tau^{k} \\
\Omega_{2}(\rho)=\widehat{\Omega}_{2}(\rho)+\widetilde{\Omega}_{2}(\rho) \text { with } \widehat{\Omega}_{2}(\rho)=\sum_{k=0}^{\widehat{N}_{2}} \widehat{\Omega}_{2 k} \rho^{k}, \\
\Omega_{1}(r)=\widehat{\Omega}_{1}(r)+\widetilde{\Omega}_{1}(r) \text { with } \widehat{\Omega}_{1}(r)=\sum_{k=0}^{\widehat{N}_{1}} \widehat{\Omega}_{1 k} r^{k}
\end{gathered}
$$

and $\widetilde{B}(\cdot), \widetilde{\Phi}_{0}(\cdot), \widetilde{\Omega}_{i}(\cdot), i=1,2$, given by $(8.2)$; cf. also Lemma 8.1 . It will be shown that these polynomials may be determined in such a way that the functions in the left-hand sides of (8.7) satisfy conditions $(2.10)$.

Lemma 8.2: The functions $B(\cdot), \Phi_{0}(\cdot), \Omega_{i}(\cdot), i=1,2$, as given by (8.7), satisfy condition (2.10) (i).

Proof: The statement of the lemma follows immediately from Lemma 8.2., note that $T>1, \rho^{+}(T)>1$ and $r^{+}(T)>1$; cf. Lemmas 3.1 and 5.1 .

For the functions $B(\cdot), \Phi_{0}(\cdot), \Omega_{i}(\cdot), i=1,2$, as described in (8.7), denote by

$$
\begin{aligned}
& F_{2}^{ \pm}(\tau) \text { the left-hand side of (4.3), } \\
& F_{1}^{ \pm}(\tau) \text { the left-hand side of (4.4). }
\end{aligned}
$$

From $(8.2),(8.7)$ and $(8.8)$ we then have (note $\omega_{20}^{(0)}=0$ ) that

$$
\begin{gathered}
F_{2}^{ \pm}(\tau)=\widehat{\Omega}_{2}\left(\rho^{ \pm}(\tau)\right)+\frac{a_{1} \tau}{\rho^{ \pm}-\tau} \widehat{B}(\tau)+\frac{1}{\tau} k_{1}\left(\rho^{ \pm}(\tau), \tau\right) \tau \widehat{\Phi}_{0}(\tau) \\
+\sum_{n=0}^{\infty} \sum_{\delta \in \mathscr{B}_{n}} \frac{1}{\tau-\tau_{\delta}^{(n)}}\left[\omega_{2 \delta}^{(n)} \frac{\tau-\tau_{\delta}^{(n)}}{\rho^{ \pm}(\tau)-\rho_{\delta}^{(n)}}\left\{\frac{\rho^{ \pm}(\tau)}{\rho_{\delta}^{(n)}}\right\}^{m_{2}}+b_{\delta}^{(n)} \frac{a_{1} \tau}{\rho^{ \pm}(\tau)-\tau}\left\{\frac{\tau}{\tau_{\delta}^{(n)}}\right\}^{m_{b}}\right. \\
\left.+\tau \phi_{\delta}^{(n)} \frac{k_{1}\left(\rho^{ \pm}(\tau), \tau\right)}{\tau}\left\{\frac{\tau}{\tau_{\delta}^{(n)}}\right\}^{m_{\phi}}\right]
\end{gathered}
$$

Note that the sum of the three terms inside the square brackets is zero for $\tau=\tau_{\delta}^{(n)},($ cf. (4.10)). For $\tau \rightarrow \infty$, it follows from (2.8), (3.16), (8.7) and (8.9), since $F_{2}^{ \pm}(\tau)$ is regular for $|\tau| \geq 1$, that 


$$
\begin{gathered}
F_{2}^{ \pm}(\tau)=\frac{\widehat{\Omega}_{2}\left(\rho^{ \pm}(\tau)\right)}{\left[\rho^{ \pm}(\tau) \widehat{N}_{2}\right.}\left[\frac{\rho^{ \pm}(\tau)}{\tau}\right]^{\widehat{N}_{2}} \tau^{\widehat{N}_{2}}+\frac{a_{1}}{R_{1}^{ \pm}-1} \frac{\widehat{B}(\tau)}{[\tau]^{\widehat{N}_{b}}} \tau^{\widehat{N}_{b}} \\
+a_{2} \pi_{2}\left(a_{1}-R_{1}^{ \pm}\right) \frac{\widehat{\Phi}_{0}(\tau)}{\left[\tau \widehat{N}_{\phi}\right.} \tau^{\widehat{N}_{\phi}+1}+\widetilde{\gamma}_{2} \tau^{\tilde{m}_{2}}+O\left(\left|\tau^{\tilde{m}_{2}-1}\right|\right), \tau \rightarrow \infty
\end{gathered}
$$

with $\tilde{\gamma}_{2}$ a nonzero constant and

Put

$$
\tilde{m}_{i}:=\max \left(m_{i}-1, m_{b}-1, m_{\phi}\right), i=1,2 .
$$

$$
\widehat{\mu}_{i}:=\max \left(\widehat{N}_{i}, \widehat{N}_{b}, \widehat{N}_{\phi}+1, m_{2}-1, m_{b}-1, m_{\phi}\right), i=1,2,
$$

with $\widehat{N}_{\phi}+1$ deleted if $\widehat{\Phi}_{0}(\cdot) \equiv 0$.

From (8.9), (8.10) and (8.12), it is readily verified that

$$
\left|F_{2}^{ \pm}(\tau)\right| \sim \tilde{\gamma}_{2}|\tau|^{\hat{\mu}_{2}} \text { for }|\tau| \rightarrow \infty
$$

Because $\tau^{ \pm}$are the only branch points of $\rho^{+}(\tau)$ and $\rho^{-}(\tau)$, it follows from (4.3) and Lemma 6.1 that $F_{2}^{+}(\tau)+F_{2}^{-}(\tau)$ is regular for all $\tau$. Consequently, Liouville's theorem implies that $F_{2}^{+}(\tau)+F_{2}^{-}(\tau)$ is a polynomial of degree $\hat{\mu}_{2}$. Such a polynomial contains $\widehat{\mu}_{2}+1$ coefficients. Because $F_{2}^{+}(\tau)+F_{2}^{-}(\tau)$ should be zero for all $\tau$, cf. (4.3), and (8.8), we thus obtain conditions for the coefficients of the polynomials $\widehat{B}(\cdot), \widehat{\Phi}_{0}(\cdot), \widehat{\Omega}_{i}(\cdot), i=1,2$, since analogous conclusions hold for $F_{1}^{ \pm}(t)$.

Note that next to these conditions we have the two conditions which stem from the definitions (2.1); see also (2.10). Further, it should be mentioned that the set of Kolmogorov equations, which are equivalent to the conditions (2.10), contains one dependent equation. So, in total, the coefficients of the polynomials have to satisfy $\widehat{\mu}_{1}+1+\widehat{\mu}_{2}+1+2-1=\widehat{\mu}_{1}+\widehat{\mu}_{2}+3$ conditions. Consequently, we have, cf. (8.7) and Lemma 8.1,

(i) $\quad m_{b} \geq \tilde{M}, m_{2} \geq \tilde{M}, m_{1} \geq \tilde{M}, m_{\phi} \geq \max (0, \tilde{M}-1)$,

(ii) $\widehat{N}_{b} \geq 0, \widehat{N}_{2} \geq 0, \widehat{N}_{1} \geq 0, \widehat{N}_{\phi} \geq 0$,

(iii) $\left|\widehat{B}_{0}\right| \geq 0,\left|\widehat{\Omega}_{20}\right| \geq 0,\left|\widehat{\Omega}_{10}\right| \geq 0,\left|\widehat{\Phi}_{00}\right| \geq 0$,

(iv) $\widehat{\mu}_{1}+\widehat{\mu}_{2}+3$ conditions have to be satisfied.

The determination of the polynomials in (8.7) for given $m_{b} m_{i}, m_{\phi}, \widehat{N}_{b}, \widehat{N}_{i}, \widehat{N}_{\phi}, i=$ 1,2 , basically proceeds as follows. From $(8.12), \widehat{\mu}_{i}, i=1,2$, is determined, so that we need $\widehat{\mu}_{2}+1$ relations to guarantee that $F_{2}^{+}(\tau) \equiv 0$, and analogously, $\widehat{\mu}_{1}+1$ relations in order that $F_{1}^{+}(t) \equiv 0$. These relations are obtained by choosing $\widehat{\mu}_{2}+1$ zero-tuples $\left(\rho+\left(\tau_{j}\right), \tau_{j}\right), j=1, \ldots, \widehat{\mu}_{2}+1$, of $h_{1}(\rho, \tau)=0$. Insertion of these zero-tuples in (4.3) leads to $\widehat{\mu}_{2}+1$ nonhomogeneous linear equations for the coefficients of the polynomials in (8.7). Analogously, $\widehat{\mu}_{1}+1$ zero-tuples $\left(r\left(t_{j}\right), t_{j}\right)$ of $h_{2}(r, t)$ are chosen and substituted in (4.4). From the structure of relations (4.3) and (4.4), it is seen that $\tau_{j}$ and $t_{j}$ may always be chosen in such a way that the resulting set of linear equations together with $\Omega_{i}(0)=\Phi_{0}(0)$, cf. $(2.10)$, is sufficient and hence leads to a solution. Once the polynomials in (8.7) have been determined, the left-hand sides in (8.7) are known. From the analysis given so far it then follows that the functions given by (8.7) satisfy (2.9) for all $|\tau|>0$. 
Obviously, we have quite some freedom in choosing the exponents in (8.2) and the degree of the polynomials in (8.7). This freedom is not so surprising because in general, a meromorphic function does not have a unique decomposition, (cf. [15], p. 304); see also Remark 8.1.

The available freedom will be used to choose the numbers in (8.14) $(i)$ and $(i i)$ as small as possible, with $\widetilde{M}$ being defined in (8.6). Before discussing this point, we first consider several zero-tuples which are most appropriate for the determination of the polynomials in (8.7).

Denote by $(\widehat{\rho}, \widehat{\tau})$ and $(\widehat{r}, \widehat{t})$ a zero-tuple of $h_{1}(\rho, \tau)=0$ and $h_{2}(r, t)=0$, respectively.

For

$$
(\widehat{\rho}, \widehat{\tau})=\left(0,-\frac{1}{a_{2}}\right) \text { and }(\widehat{r}, \widehat{t})=\left(0,-\frac{1}{a_{1}}\right)
$$

it follows from (2.9) that

(i) $\quad \Omega_{2}(0)-a_{1} B\left(-\frac{1}{a_{2}}\right)-\left(1+a_{1} \pi_{2}\right) \Phi_{0}\left(-\frac{1}{a_{2}}\right)=0$,

(ii) $\quad \Omega_{1}(0)+a_{2} B\left(-\frac{1}{a_{1}}\right)-\left(1+a_{2} \pi_{1}\right) \Phi_{0}\left(-\frac{1}{a_{1}}\right)=0$.

Note that $\tau=-\frac{1}{a_{2}}>\tau_{0}^{(0)}=T$ and $\tau=-\frac{1}{a_{1}}>\tau_{0}^{(0)}=T$, so that $\tau=-\frac{1}{a_{2}}$ and $\tau=$ $-\frac{1}{a_{1}}$ are not poles of $B(\cdot)$ and of $\Phi_{0}(\cdot)$; cf. Lemmas 6.1 and 6.2. From definitions (2.1), we have

Hence, from (8.16),

$$
\Omega_{i}(0)=\Phi_{0}(0), \quad i=1,2
$$

(i) $\quad a_{1} B\left(-\frac{1}{a_{2}}\right)+\left(1+a_{1} \pi_{2}\right) \Phi_{0}\left(-\frac{1}{a_{2}}\right)-\Phi_{0}(0)=0$,

(ii) $\quad-a_{2} B\left(-\frac{1}{a_{1}}\right)+\left(1+a_{2} \pi_{1}\right) \Phi_{0}\left(-\frac{1}{a_{1}}\right)-\Phi_{0}(0)=0$.

Comparison of the relations (8.18) with (2.1) (iv) shows that

For

$$
\begin{aligned}
& E\left\{r^{\mathbf{x}_{1}}\left(\mathbf{x}_{1}=\mathbf{x}_{2}+1\right\} \text { is finite for } r=-\frac{1}{a_{1}},\right. \\
& E\left\{r^{\mathbf{x}_{2}}\left(\mathbf{x}_{2}=\mathbf{x}_{1}+1\right\} \text { is finite for } r=-\frac{1}{a_{2}} .\right.
\end{aligned}
$$

we have

$$
(\widehat{\rho}, \widehat{\tau})=(0,0) \text { and }(\widehat{r}, \widehat{t})=(0,0)
$$

and so, from (2.9)

$$
\left.\frac{d \tau}{d \rho}\right|_{\substack{\tau=0 \\ \rho=0}}=0,\left.\frac{d t}{d r}\right|_{\substack{t=0 \\ r=0}}=0
$$

(i) $\quad \Omega_{2}(0)+a_{1} B(0)-\Phi_{0}(0)=0$,

(ii) $\quad \Omega_{1}(0)-a_{2} B(0)-\Phi_{0}(0)=0$.

Hence, from (8.17) and (8.21) $(i)$ or (8.21) $(i i)$,

$$
B(0)=0 \text {. }
$$

Obviously, here the dependency of the set of Kolmogorov equations is manifested.

For

$$
(\widehat{\rho}, \widehat{\tau})=\left(1, \frac{1}{a_{1}}\right) \text { and }(\widehat{r}, \widehat{t})=\left(1, \frac{1}{a_{2}}\right)
$$

we have from (2.9) 
(i) $\quad \Omega_{2}(1)+\frac{a_{1}}{a_{1}-1} B\left(\frac{1}{a_{1}}\right)-\Phi_{0}\left(\frac{1}{a_{1}}\right)=0, a_{1} \neq 1$,

(ii) $\quad \Omega_{1}(1)-\frac{a_{2}}{a_{2}-1} B\left(\frac{1}{a_{2}}\right)-\Phi_{0}\left(\frac{1}{a_{2}}\right)=0, a_{2} \neq 1$.

For

$$
(\widehat{\rho}, \widehat{\tau})=(1,1) \text { and }(\widehat{r}, \widehat{t})=(1,1)
$$

we have from (2.9),

$$
\begin{aligned}
& \Omega_{2}(1)+a_{1} \lim _{\rho \rightarrow \tau=1} \frac{B(\tau)}{\rho-\tau}+\left[-1+a_{2} \pi_{2}\left(a_{1}-1\right)\right] \Phi_{0}(1)=0 \\
& \Omega_{1}(1)-a_{2} \lim _{r \rightarrow t=1} \frac{B(t)}{r-t}+\left[-1+a_{1} \pi_{1}\left(a_{2}-1\right)\right] \Phi_{0}(1)=0 .
\end{aligned}
$$

Hence, since $\Omega_{i}(1)$ is finite,

From (3.7) we have

$$
B(1)=0
$$

Hence, from (8.26),

$$
\frac{d \rho}{d t}-1=\frac{-a_{1}}{a_{1} a_{2}+a_{1}-a_{2}}, \frac{d r}{d t}-1=\frac{-a_{2}}{a_{1} a_{2}+a_{1}-a_{2}} .
$$

$$
\begin{aligned}
& \Omega_{2}(1)-\left.\left(a_{1} a_{2}+a_{1}-a_{2}\right) \frac{d}{d \tau} B(\tau)\right|_{\tau=1}+\left[-1+a_{2} \pi_{2}\left(a_{1}-1\right)\right] \Phi_{0}(1)=0, \\
& \Omega_{1}(1)+\left.\left(a_{1} a_{2}+a_{1}-a_{2}\right) \frac{d}{d \tau} B(\tau)\right|_{\tau=1}+\left[-1+a_{1} \pi_{1}\left(a_{2}-1\right)\right] \Phi_{0}(1)=0 .
\end{aligned}
$$

Next we consider the zero-tuples, cf. (3.4),

$$
(\widehat{\rho}, \widehat{\tau})=\left(\rho\left(\tau^{+}\right), \tau^{+}\right) \text {and }(\widehat{r}, \widehat{t})=\left(r\left(t^{+}\right), t^{+}\right) .
$$

From (3.4), it is seen that $\rho\left(\tau^{+}\right)$is a zero with multiplicity two of $h_{1}\left(\rho, \tau^{+}\right)$. Consequently, it follow from (4.3) that $\rho\left(\tau^{+}\right)$should be a zero of multiplicity two of (4.3) with $\tau=\tau^{+}$, since $\tau=\tau^{+}$is not a pole of $B(\tau)$ and $\Phi_{0}(\tau)$, and $\rho=\rho\left(\tau^{+}\right)$is not a pole of $\Omega_{2}(\rho)$. Hence, from (2.9):

(ii) $\quad\left[\frac{d}{d \rho} \Omega_{2}(\rho)+B\left(\tau^{+}\right) \frac{d}{d \rho} \frac{a_{1} \tau^{+}}{\rho-\tau^{+}}+\Phi_{0}\left(\tau^{+}\right) \frac{d}{d \rho} k_{1}\left(\rho, \tau^{+}\right)\right]_{r=\rho\left(\tau^{+}\right)}=0$

(iii) $\left[\Omega_{1}(r)-\frac{a_{2} t^{+}}{r-t^{+}} B\left(t^{+}\right)+k_{2}\left(r, t^{+}\right) \Phi_{0}\left(t^{+}\right)\right]_{r=r\left(t^{+}\right)}=0$

(iv) $\left[\frac{d}{d r} \Omega_{1}(r)-B\left(\tau^{+}\right) \frac{d}{d r} \frac{a_{2} t^{+}}{r-t^{+}}+\Phi_{0}\left(t^{+}\right) \frac{d}{d r} k_{2}\left(r, t^{+}\right)\right]_{r=r\left(t^{+}\right)}=0$.

Note that $\rho\left(\tau^{+}\right)-\tau^{+} \neq 0$.

Next note that $(2.4)(i)$ for $r_{2}=0, r_{1} \neq 0$, leads to (8.17) and so the zero-tuple $(\widehat{\rho}, \widehat{\tau})=\left(\rho\left(\tau^{-}\right), \tau^{-}\right)=(0,0)$ needs no further attention.

Finally, we consider the zero-tuples, cf. (3.4),

$$
(\widehat{\rho}, \widehat{\tau})=\left(\rho^{ \pm}, \tau\left(\rho^{ \pm}\right)\right) \text {and }(\widehat{r}, \widehat{t})=\left(r^{ \pm}, t\left(r^{ \pm}\right)\right) .
$$

For $\rho=\rho^{+}$it is seen from (3.4) that $\widehat{\tau}=\tau\left(\rho^{+}\right)$is a zero of multiplicity two of $h_{1}\left(\rho^{+}, \tau\right)$; also, $\widehat{\tau}=\tau\left(\rho^{-}\right)$is a zero of multiplicity two of $h_{1}\left(\rho^{-}, \tau\right)$. As before, we 
obtain from (2.9): for $\rho^{+} \neq 1$,

$$
\begin{aligned}
& \text { (i) }\left[\Omega_{2}\left(\rho^{ \pm}\right)+\frac{a_{1} \tau}{\rho^{ \pm}-\tau} B(\tau)+k_{1}\left(\rho^{ \pm}, \tau\right) \Phi_{0}(\tau)\right]_{\tau=\tau\left(\rho^{ \pm}\right)}=0, \\
& \text { (ii) }\left[\frac{d}{d \tau}\left\{\frac{a_{1} \tau}{\rho^{ \pm}-\tau} B(\tau)\right\}+\frac{d}{d \tau}\left\{k_{1}\left(\rho^{ \pm}, \tau\right) \Phi_{0}\right\}\right]_{\tau=\tau\left(\rho^{ \pm}\right)}=0,
\end{aligned}
$$

and for $r^{+} \neq 1$,

(i) $\quad\left[\Omega_{1}\left(r^{ \pm}\right)-\frac{a_{2} t}{r^{ \pm}-t} B(t)+k_{2}\left(r^{ \pm}, t\right) \Phi_{0}(t)\right]_{t=t\left(r^{ \pm}\right)}=0$

(ii) $\left.\quad \frac{d}{d t}\left\{{\frac{-a_{2} t}{r^{ \pm}-t}}_{-t} B(t)\right\}+\frac{d}{d t}\left\{k_{2}\left(r^{ \pm}, t\right) \Phi_{0}(t)\right\}\right]_{t=t\left(r^{ \pm}\right)}=0$.

Note that, cf. (3.4),

$$
\rho^{+}=1 \Leftrightarrow a_{1}=1 \text { and } r^{+}=1 \Leftrightarrow a_{2}=1 \text {. }
$$

The case $a_{1}=1$ has to be excluded from (8.24) $(i)$ and (8.30) (i), similarly $a_{2}=1$ from (8.24) $(i i)$ and (8.30) (ii). If $a_{1}=1$, then the second terms in (8.24) $(i)$ and (8.30) $(i)$ have to replaced by their limits for $a_{1} \rightarrow 1$.

We proceed with the determination of the polynomials in (8.7). With regard to the available freedom mentioned above we shall try to choose the degrees of the polynomials in (8.7) as small as possible.

First we consider the case

$$
\tilde{M}=1 \text {. }
$$

Take for the present case

$$
\begin{gathered}
m_{b}-1=m_{2}-1=m_{1}-1=m_{\phi}=\widehat{N}_{b}=\widehat{N}_{2}=\widehat{N}_{1}=\widehat{N}_{\phi}=0, \\
\widehat{B}_{0}=0, \widehat{\Phi}_{00}=0 .
\end{gathered}
$$

This choice is consistent with (8.14), and it follows from (8.2) and (8.12) that

(i) $\widetilde{B}(0)=\widetilde{\Omega}_{2}(0)=\widetilde{\Omega}_{1}(0)=0, \widetilde{\Phi}_{0}(0)=-\sum_{n=0}^{\infty} \sum_{\delta \in \mathscr{B}_{n}} \frac{\phi \delta}{\tau_{\delta}^{(n)}}$.

(ii) $\widehat{\mu}_{1}=\widehat{\mu}_{2}=0$.

From (8.37) $(i i)$, it is seen that we need three coefficients. From (8.8), (8.17), (8.22) and (8.37), we obtain

$$
\widehat{\Omega}_{i}(0)=\widehat{\Omega}_{i 0}=\widetilde{\Phi}_{0}(0), i=1,2 ;
$$

and so the three nonzero coefficients of the polynomials in (8.8) have been determined. The results so far obtained lead to the following.

Theorem 8.1: For $\frac{1}{a_{1}}+\frac{1}{a_{1}}>1, a_{1} \neq a_{2}, \widetilde{M}=1$, cf. (8.6), the functions $B(\cdot)$, $\Phi_{0}(\cdot), \Omega_{i}(\cdot), i=1,2$, which satisfy the conditions (2.10), are given by, cf. (8.2),

(i) $\quad B(\tau)=\sum_{n=0}^{\infty} \sum_{\delta \in \mathscr{B}_{n}} \frac{b_{\delta}^{(n)}}{\tau-\tau \delta^{(n)}} \frac{\tau}{\tau_{\delta}^{(n)}}, \quad|\tau|<T=\left(\frac{1}{a_{1}}+\frac{1}{a_{2}}\right)^{2}$,

(ii) $\Phi_{0}(\tau)=\sum_{n=0}^{\infty} \sum_{\delta \in \mathscr{B}_{n}} \frac{\phi_{\delta}^{(n)}}{\tau-\tau \delta^{(n)}}, \quad|\tau|<T$,

(iii) $\Omega_{2}(\rho)=-\sum_{n=0}^{\infty} \sum_{\delta \in \mathscr{B}_{n}} \frac{\phi_{\delta}^{(n)}}{\tau\left(^{(n)}\right.}+\sum_{n=1}^{\infty} \sum_{\delta \in \mathscr{B}_{n}} \frac{\omega_{2 \delta}^{(n)}}{\rho-\rho_{\delta}^{(n)}} \frac{\rho}{\rho \delta},|\rho|<\rho+(T)$, 
(iv) $\quad \Omega_{1}(r)=-\sum_{n=0}^{\infty} \sum_{\delta \in \mathscr{B}_{n}} \frac{\phi \delta^{(n)}}{\tau \delta^{(n)}}+\sum_{n=1}^{\infty} \sum_{\delta \in \mathscr{B}_{n}} \frac{\omega_{1 \delta}^{(n)}}{r-r_{\delta}^{(n)}} \frac{r}{r(n)},|r|<r+(T)$.

These functions have meromorphic continuations throughout the whole complex plane, which are given by the right-hand sides of $(8.39)(i) \ldots(i v)$. The residues $b_{\delta}^{(n)}$, $\phi_{n}^{(n)}, \omega_{i \delta}^{(n)}, i=1,2$ can be calculated recursively (see Lemma 6.2), they all contain the factor $\phi_{0}\left(\tau_{0}^{(0)}\right)$, which is uniquely determined by

$$
\frac{1}{a_{1}} \Omega_{2}(1)+\frac{1}{a_{2}} \Omega_{1}(1)=\frac{1}{a_{1}}+\frac{1}{a_{2}}-1 \text {. }
$$

The generating functions $E\left\{r^{\mathbf{x}_{1}}\left(\mathbf{x}_{1}=\mathbf{x}_{2}+1\right)\right\}, \quad E\left(r^{\mathbf{x}_{2}}\left(\mathbf{x}_{2}=\mathbf{x}_{1}+1\right)\right\}, \quad|r|<$

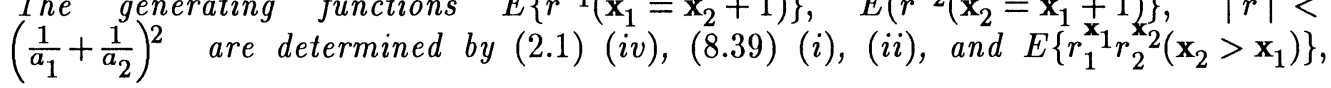
$E\left\{r_{1}^{\mathbf{x}_{1}}, r_{2}^{\mathbf{x}_{2}}\left(\mathbf{x}_{1}>\mathbf{x}_{2}\right)\right\}$ are obtained from (2.4) and (8.39).

The queue length process $\left\{\mathbf{x}_{1}(t), \mathbf{x}_{2}(t), t>0\right\}$ is positive recurrent if and only if $\frac{1}{a_{1}}+\frac{1}{a_{2}}>1$.

Proof: From (8.35)-(8.38) and the analysis given above, it follows that the functions in (8.39) satisfy (4.3) for all $|\tau| \geq 0$ and (4.4) for all $|t| \geq 0$. So, by using Lemma 8.2, they satisfy conditions $(2.10)(i)$ and $(2.9)$ or $(2.10)(i i)$. The determination of $E\left\{r^{\mathbf{x}_{1}}\left(\mathbf{x}_{1}=\mathbf{x}_{2}+1\right)\right\}$ follows from (2.1) (iv) and (8.39). It is further seen, cf. (8.19), that the radius of convergence of the latter generating function is larger than one, analogously, for $E\left\{r^{\mathbf{x}_{2}}\left(\mathbf{x}_{2}=\mathbf{x}_{1}+1\right)\right\}$. From (2.4) and (8.39), the bivariate generating functions $E\left\{r_{1}^{\mathbf{x}_{1}} r_{2}^{\mathbf{x}_{2}}\left(\mathbf{x}_{2}>\mathbf{x}_{1}\right)\right\}$ and $E\left\{r_{1}^{\mathbf{x}_{1}} r_{2}^{\mathbf{x}_{2}}\left(\mathbf{x}_{1}>\mathbf{x}_{2}\right)\right\}$ are obtained. It is readily seen that the domain of convergence of these bivariate generating functions contains the Cartesian product unit disks $\left|r_{1}\right| \leq 1,\left|r_{2}\right| \leq 1$, as a true subset. Hence, the coefficients in the series expansions of the bivariate generating function $E\left\{r_{1}^{\mathbf{x}_{1}} r_{2}^{\mathbf{x}_{2}}\right\}$ is an absolutely convergent solution of the Kolmogorov equations. Hence, Foster's criterion, cf. Remark 2.2, implies that the queue length process $\left\{\mathbf{x}_{1}(t), \mathbf{x}_{2}(t), t>0\right\}$ is positive recurrent for $\frac{1}{a_{1}}+\frac{1}{a_{2}}>1 ;(2.6)$ shows that this condition is also necessary. Because all generating functions contain $\phi_{0}(T)$ as a linear factor, cf. Lemma 6.1, this factor follows from (8.40), cf. (2.1) and (2.5), and so it is uniquely defined. For the uniqueness of the solution constructed for the conditions of the theorem, see Remark 2.2.

Next, we consider the case

Take

$$
\tilde{M}=2, \frac{1}{a_{1}}+\frac{1}{a_{2}}>1, a_{1} \neq a_{2} .
$$

$$
\begin{gathered}
m_{b}-1=m_{2}-1=m_{1}-1=m_{\phi}=1 \\
\widehat{N}_{b}=1, \quad \widehat{N}_{2}=\widehat{N}_{1}=\widehat{N}_{\phi}=0 .
\end{gathered}
$$

This choice is again consistent with (8.14) and it follows that

$$
\widehat{\mu}_{1}=\widehat{\mu}_{2}=1 \text {. }
$$

Hence, we need four coefficients. We have $\widehat{N}_{b}=1$ and, further, $\widehat{\Phi}_{00}$ should be nonzero since $\widetilde{\Phi}_{0}(0)=0$, and $\Phi_{0}(0)$ should be positive for a positive recurrent queue length process; cf. $(2.1)(i)$. Note $\widetilde{B}(0)=0$ for $m_{b}=1$, so that $\widehat{B}_{0}(0)=0$; cf. (8.17). From the two equations (8.8), $\widehat{B}_{1}$ and $\widehat{\Phi}_{00}$ can be determined, their main determinant is nonzero. Then, from (8.17) we obtain $\widehat{\Omega}_{i 0}, i=1,2$. The explicit equations for 
$\widehat{B}_{1}$ and $\widehat{\Phi}_{00}$ read, cf. $(8.18)$,

$$
\begin{gathered}
-\frac{a_{1}}{a_{2}} \widehat{B}_{1}+a_{1} \pi_{2} \widehat{\Phi}_{00}=-a_{1} \widetilde{B}\left(-\frac{1}{a_{2}}\right)-\left(1+a_{1} \pi_{2}\right) \widetilde{\Phi}_{0}\left(-\frac{1}{a_{2}}\right) \\
+\frac{a_{2}}{a_{1}} \widehat{B}_{1}+a_{2} \pi_{1} \widehat{\Phi}_{00}=a_{2} \widetilde{B}\left(-\frac{1}{a_{1}}\right)-\left(1+a_{2} \pi_{1}\right) \widetilde{\Phi}_{0}\left(-\frac{1}{a_{1}}\right) .
\end{gathered}
$$

Hence, with $\widehat{B}_{1}$ and $\widehat{\Phi}_{00}$ determined by (8.44) we have for the present case (8.41):

$$
\begin{gathered}
B(\tau)=\tau \widehat{B}_{1}+\sum_{n=0}^{\infty} \sum_{\delta \in \mathscr{B}_{n}} \frac{b_{\delta}^{(n)}}{\tau-\tau_{\delta}^{(n)}}\left[\frac{\tau}{\tau_{\delta}^{(n)}}\right]^{2}, \\
\Phi(\tau)=\widehat{\Phi}_{00}+\sum_{n=0}^{\infty} \sum_{\delta \in \mathscr{B}_{n}} \frac{\phi_{\delta}^{(n)}}{\tau-\tau_{\delta}^{(n)}} \frac{\tau}{\tau \delta^{(n)}}, \\
\Omega_{2}(\rho)=\widehat{\Phi}_{00}+\sum_{n=1}^{\infty} \sum_{\delta \in \mathscr{B}_{n}} \frac{\omega_{2 \delta}^{(n)}}{\rho-\rho_{\delta}^{(n)}}\left[\frac{\rho}{\rho_{\delta}^{(n)}}\right]^{2}, \\
\Omega_{1}(r)=\widehat{\Phi}_{00}+\sum_{n=1}^{\infty} \sum_{\delta \in \mathscr{B}_{n}} \frac{\omega_{1 \delta}^{(n)}}{r-r_{\delta}^{(n)}}\left[\frac{r}{r_{\delta}^{(n)}}\right]^{2} .
\end{gathered}
$$

Note that (8.27) implies

$$
\widehat{B}_{1}=-\sum_{n=0}^{\infty} \sum_{\delta \in \mathfrak{B}_{n}} \frac{b_{\delta}^{(n)}}{1-\tau_{\delta}^{(n)}} \frac{1}{\left(\tau_{\delta}^{(n)}\right)^{2}} .
$$

For conditions (8.41) and with (8.39) replaced by (8.45) in Theorem 8.1 we obtain a relevant theorem for the case $\widetilde{M}=2, a_{1} \neq a_{2}, \frac{1}{a_{1}}+\frac{1}{a_{2}}>1$. It is fully analogous to Theorem 8.1 and its explicit formulation is, therefore, omitted.

The determination of the polynomials in $(8.7)$ for $\widetilde{M} \geq 3, \frac{1}{a_{1}}+\frac{1}{a_{1}}>1, a_{1} \neq a_{2}$, proceeds along the same lines as for the cases $\tilde{M}=1,2$. The relations $(8.17),(8.18)$, (8.22), (8.24), (8.27), (8.28), (8.30), (8.32) and (8.34) yield, in general, twenty-two equations, except for $a_{1}=1$ or $a_{2}=1$, cf. (8.24), (8.28) and (8.34), so their number suffices for rather large $\tilde{M}$; cases with $\tilde{M} \geq 3$ seem hardly to occur in the applications.

Remark 8.1: The degrees of the polynomials and the exponents of the meromorphic functions have been introduced in (8.7) and (8.2). They have to be determined in such a way that (8.14) is satisfied and $F_{i}^{+}(\tau)+F_{i}^{-}(\tau), i=1,2$ are zero at $\widehat{\mu}_{i}+1$ points. In this determination there is no objection replacing $m_{b}$ by $m_{b}+h_{b}, m_{\phi}$ by ${\underset{\sim}{m} \phi}_{\phi}+h_{\phi}, m_{2}$ by $m_{2}+h_{2}$ and $m_{1}$ by $m_{1}+h_{1}$, with $h_{b}, h_{\phi}, h_{2}, h_{1}$, positive integers (and $\tilde{M}$ defined by (8.6)). Such a change when compared with the case that $h_{b}, h_{\phi}, h_{2}, h_{1}$ are all zero, actually amounts to subtraction of a polynomial from the meromorphic function and addition of that polynomial to the " $\wedge$ " polynomial; see (8.7). In fact, this also occurs by noting that the solution given by (8.44) also holds for the case $\tilde{M}=1$.

Remark 8.2: From (8.2) and (8.7), it is readily seen that $T=\tau_{0}^{(0)}=\left(\frac{1}{a_{1}}+\frac{1}{a_{2}}\right)^{2}$ $>1$ is the smallest pole of $\Phi_{0}(\cdot)$ and also of $B(\cdot)$. Hence, $T$ determines the asymptotic behavior of $\operatorname{Pr}\left\{\mathbf{x}_{1}=\mathbf{x}_{2}=n\right\}$ for $n \rightarrow \infty$, i.e.

Similarly, it is seen that

$$
\operatorname{Pr}\left\{\mathbf{x}_{1}=\mathbf{x}_{2}=n\right\} \sim-\frac{\phi_{0}(T)}{T^{n+1}} \text { for } n \rightarrow \infty .
$$




$$
\begin{aligned}
& \rho_{0}^{(1)}=\rho^{+}\left(\tau_{0}^{(0)}\right)=a_{1}\left(\frac{1}{a_{1}}+\frac{1}{a_{2}}\right)^{3}+\frac{a_{1}}{a_{2}}\left(\frac{1}{a_{1}}+\frac{1}{a_{2}}\right), \\
& r_{0}^{(1)}=r^{+}\left(\tau_{0}^{(0)}\right)=a_{2}\left(\frac{1}{a_{1}}+\frac{1}{a_{2}}\right)^{3}+\frac{a_{2}}{a_{1}}\left(\frac{1}{a_{1}}+\frac{1}{a_{2}}\right),
\end{aligned}
$$

are the smallest poles of $\Omega_{2}(\cdot)$ and $\Omega_{1}(\cdot)$, respectively, and so they determine the leading term in the asymptotic behavior of $\operatorname{Pr}\left\{\mathbf{x}_{2}=n, \mathbf{x}_{1}=0\right\}$ and $\operatorname{Pr}\left\{\mathbf{x}_{1}=n, \mathbf{x}_{2}=0\right\}$ for $n \rightarrow \infty$.

Remark 8.4: Numerical calculations indicate that always $\left|\lambda_{1} \mu_{1}+\lambda_{2} \mu_{2}\right|>1$. For quite a few cases this has been proved in Appendix C. However, a complete proof of $\widetilde{M} \geq 1$ has not been obtained; actually, this is not very important, because if $\widetilde{M}=0$, then Theorem 8.1 also applies. Note that then the sum in (8.39) (i) may be written as

$$
\sum_{n=0}^{\infty} \sum_{\delta \in \mathscr{B}_{n}} \frac{b_{\delta}^{(n)}}{\tau-\tau_{\delta}^{(n)}} \frac{\tau}{\tau_{\delta}^{(n)}}=\sum_{n=0}^{\infty} \sum_{\delta \in \mathscr{B}_{n}} \frac{b_{\delta}^{(n)}}{\tau_{\delta}^{(n)}}+\sum_{n=0}^{\infty} \sum_{\delta \in \mathscr{B}_{n}} \frac{b_{\delta}^{(n)}}{\tau-\tau_{\delta}^{(n)}},
$$

since for $\tilde{M}=0$, the first sum in the right-hand side converges absolutely and the second sum is a well-defined meromorphic function, analogously for the other sums in (8.39).

\section{The Solution for the Case $a_{1}=a_{2}<2, \pi_{1} \neq \pi_{2}$}

In the preceding section, the solution has been described for the case $a_{1} \neq a_{2}$. In this section, we derive the solution for the case

$$
a:=a_{1}=a_{2}<2, \pi_{1} \neq \pi_{2}, 0<\pi_{1}=1-\pi_{2}<1 .
$$

Again, $T$ is defined as in Section 5, so cf. Lemma 5.1:

$$
T=\frac{4}{a^{2}}, \rho^{-}(T)=r^{-}(T)=\frac{2}{a}
$$

note that (9.1) implies $h_{1}(\rho, \tau)=h_{2}(\rho, \tau)$, so

$$
r^{ \pm}(\tau)=\rho^{ \pm}(\tau), \tau^{ \pm}(\rho)=t^{ \pm}(\rho) .
$$

Again, Assumption 5.1 is here made, and, as in (5.9), it follows that

$$
\tau=T \text { is a simple pole of } B(\tau) \text { and also of } \Phi_{0}(\tau) \text {. }
$$

It follows, cf. the derivations of (5.10), that

$$
\begin{aligned}
& \frac{a T}{\rho^{-}(T)-T} b(T)+k_{1}\left(\rho^{-}(T), T\right) \phi_{0}(T)=0, \\
& \frac{-a T}{\rho^{-}(T)-T} b(T)+k_{2}\left(\rho^{-}(T), T\right) \phi_{0}(T)=0,
\end{aligned}
$$

and that the two relations in (9.5) are linearly dependent, because (9.2) implies that the main determinant of the system (9.5) is equal to zero.

From (9.1) and (9.3), it is seen that the zero-tuple of the ladder (3.8) generated by the zero-tuple $T$ on $h_{1}(\rho, \tau)$ induces on $h_{2}(r, t)=0$ ladders, which are all congruent to ladder, cf. Figure 3.2,

$$
\begin{gathered}
\left(\rho_{\nu}, \tau_{\nu}\right), \ldots,\left(\rho_{n-1}, \tau_{n-1}\right),\left(\rho_{n}, \tau_{n}\right),\left(\rho_{n+1}, \tau_{n+1}\right), \ldots \\
\tau_{0}:=T, \rho_{-}:=\rho^{-}(T)
\end{gathered}
$$


with $\nu$ as defined below (3.9), and $\rho_{n}, \tau_{n}$ recursively defined as in (3.9).

Consider for the present case the relations (6.2) and (6.4), i.e.,

$$
\begin{gathered}
\frac{a T}{\rho_{1}-T} b(T)+k_{1}\left(\rho_{1}, T\right) \phi_{0}(T)+\omega_{2}\left(\rho_{1}\right)\left[\frac{d \rho+(\sigma)}{d \sigma}\right]_{\sigma=T}^{-1}=0 \\
\frac{a T}{\rho_{0}-T} b(T)+k_{2}\left(\rho_{0}, T\right) \phi_{0}(T)=0 .
\end{gathered}
$$

As in Section 6, cf. (6.5), it follows that

$$
\rho_{1} \text { is a simple pole of } \Omega_{i}(\rho), 0<\left|\omega_{i}\left(\rho_{1}\right)\right|<\infty, i=1,2 .
$$

Next, consider for the present case the relations (4.9) $(i)$ and (4.10) $(i)$,

$$
\begin{aligned}
& \omega_{2}\left(\rho_{1}\right)+\left[\frac{a \tau_{1}}{\rho_{1}-\tau_{1}} b\left(\tau_{1}\right)+k_{1}\left(\rho_{1}, \tau_{1}\right) \phi_{0}\left(\tau_{1}\right)\right]\left[\frac{d \tau^{+}(\sigma)}{d \sigma}\right]_{\sigma=\rho_{1}}^{-1}=0, \\
& \omega_{1}\left(\rho_{1}\right)+\left[\frac{-a \tau_{1}}{\rho_{1}-\tau_{1}} b\left(\tau_{1}\right)+k_{2}\left(\rho_{1}, \tau_{1}\right) \phi_{0}\left(\tau_{1}\right)\right]\left[\frac{d \tau^{+}(\sigma)}{d \sigma}\right]_{\sigma=\rho_{1}}^{-1}=0 .
\end{aligned}
$$

Via (9.7), $\omega_{2}\left(\rho_{1}\right)$ can be expressed uniquely as a linear function of $\phi_{0}(T)$; note that the determinant formed by the coefficients of $b(T)$ and $\phi_{0}(T)$ is nonzero, cf. (5.13), (9.5), and that $\rho_{0}=\rho^{-}(T)$. Analogously, $\omega_{1}\left(\rho_{1}\right)$ is determined. It is now readily seen that system (9.9) for the unknowns $b\left(\tau_{1}\right)$ and $\phi_{0}\left(\tau_{1}\right)$ has a solution $b\left(\tau_{1}\right) \neq 0$, $\phi_{0}\left(\tau_{1}\right) \neq 0$, since its main determinant is nonzero; cf. (5.13) for $a_{1}=a_{2}, \pi_{1} \neq \pi_{2}$. Consequently,

$$
\tau_{1} \text { is a simple pole of } B(\tau) \text { and also of } \Phi_{0}(\tau) \text {. }
$$

Next, we consider relations (4.10) and (4.11) for the present case, i.e.,

$$
\begin{aligned}
& \omega_{2}\left(\rho_{2}\right)\left[\frac{d \rho+(\sigma)}{d \sigma}\right]_{\sigma=\tau_{1}}^{-1}+\frac{a \tau_{1}}{\rho_{2}-\tau_{1}} b\left(\tau_{1}\right)+k_{1}\left(\rho_{2}, \tau_{1}\right) \phi_{0}\left(\tau_{1}\right)=0, \\
& \omega_{1}\left(\rho_{2}\right)\left[\frac{d \rho+(\sigma)}{d \sigma}\right]_{\sigma=\tau_{1}}^{-1}+\frac{-a \tau_{1}}{\rho_{2}-\tau_{1}} b\left(\tau_{1}\right)+k_{1}\left(\rho_{2}, \tau_{1}\right) \phi_{0}\left(\tau_{1}\right)=0 .
\end{aligned}
$$

Hence, since $b\left(\tau_{1}\right)$ and $\phi_{0}\left(\tau_{1}\right)$ are determined by $(9.9)$, it readily follows that $\omega_{2}\left(\rho_{2}\right)$ and $\omega_{1}\left(\rho_{2}\right)$ are both finite and nonzero. Consequently,

$$
\rho_{2} \text { is a simple pole of } \Omega_{i}(\rho), i=1,2 .
$$

By repeating the argumentation above, it is readily verified that the following lemma holds; its detailed proof is therefore omitted.

Lemma 9.1: For the case $a_{1}=a_{2}, \pi_{1} \neq \pi_{2}$ with $\tau_{0}=T$ :

(i) $\quad \tau_{n}, n=0,1,2, \ldots$, are simple poles of $B(\tau)$ and also of $\Phi_{0}(\tau)$;

(ii) $\quad \rho_{n}, n=1,2, \ldots$, are simple poles of $\Omega_{i}(\rho), i=1,2$;

(iii) the residues $b\left(\tau_{n}\right), \phi_{0}\left(\tau_{n}\right), \omega_{i}\left(\rho_{n}\right), i=1,2$, are recursively determined by

$$
\frac{a T}{\rho_{0}-T} b(T)+k_{1}\left(\rho_{0}, T\right) \phi_{0}(T)=0
$$

and for $n=1,2, \ldots$, by

$$
\omega_{2}\left(\rho_{n}\right)+\left[\frac{a \tau_{n-1}}{\rho_{n}-\tau n-1} b\left(\tau_{n-1}\right)+k_{1}\left(\rho, \tau_{n-1}\right) \phi_{0}\left(\tau_{n-1}\right)\right]\left[\frac{d \tau-(\sigma)}{d \sigma}\right]_{\sigma=\rho_{0}}^{-1}=0
$$


(ii) $\quad \omega_{1}\left(\rho_{n}\right)+\left[\frac{-a \tau_{n-1}}{\rho_{n}-\tau_{n-1}} b\left(\tau_{n-1}\right)+k_{2}\left(\rho, \tau_{n-1}\right) \phi_{0}\left(\tau_{n-1}\right)\right]\left[\frac{d \tau^{-}(\sigma)}{d \sigma}\right]_{\sigma=\rho_{n}}^{-1}$

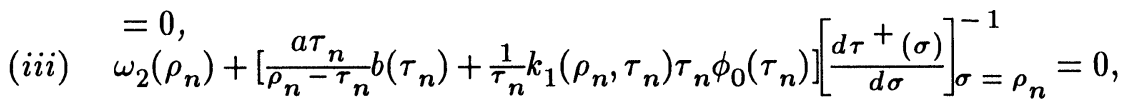

(iv) $\quad \omega_{1}\left(\rho_{n}\right)+\left[\frac{-a \tau_{n}}{\rho_{n}-\tau_{n}} b\left(\tau_{n}\right)+\frac{1}{\tau_{n}} k_{2}\left(\rho_{n}, \tau_{n}\right) \tau_{n} \phi_{0}\left(\tau_{n}\right)\right]\left[\frac{d \tau+(\sigma)}{d \sigma}\right]_{\sigma=\rho_{n}}^{-1}=0$.

Next we consider the asymptotic behavior of $b\left(\tau_{n}\right), \phi_{n}\left(\tau_{n}\right)$ and $\omega_{i}\left(\rho_{n}\right), i=1,2$, for $n \rightarrow \infty$.

Lemma 9.2: For the case $a_{1}=a_{2}=a, \pi_{1} \neq \pi_{2}$ with $\tau_{0}=T$ :

$$
\begin{gathered}
\psi:=\lim _{n \rightarrow \infty} \frac{b\left(\tau_{n+1}\right)}{b\left(\tau_{n}\right)}=\lim _{n \rightarrow \infty} \frac{\phi_{0}\left(\tau_{n+1}\right)}{\phi_{0}\left(\tau_{n}\right)}=\lim _{n \rightarrow \infty} \frac{\omega_{i}\left(\rho_{n+1}\right)}{\omega_{i}\left(\rho_{n}\right)}, i=1,2, \\
\psi=\frac{R^{-}-1}{R^{+}-1} \frac{R^{+}}{R^{-}}=\lambda^{-1} \frac{R^{-}-1}{R^{+}-1}<0
\end{gathered}
$$

with (since $\left.a_{1}=a_{2}\right)$,

$$
R_{2}^{-}=R_{1}^{-} \equiv R^{-}, R_{2}^{+}=R_{1}^{+} \equiv R^{+}, \lambda=\lambda_{1}=\lambda_{2}=\frac{R^{-}}{R^{+}} \text {. }
$$

Proof: Add (9.14) $(i)$ and (ii) and also (9.14) (iii) and (iv). Next, eliminate from the resulting expressions $\omega_{2}\left(\rho_{n}\right)+\omega_{1}\left(\rho_{n}\right)$; this leads to

$$
\begin{gathered}
{\left[k_{1}\left(\rho_{n}, \tau_{n}\right)+k_{2}\left(\rho_{n}, \tau_{n}\right)\right] \phi_{0}\left(\tau_{n}\right)\left[\frac{d \tau^{+}(\sigma)}{d \sigma}\right]_{\sigma=\rho_{n}}^{-1}} \\
=\left[k_{1}\left(\rho_{n}, \tau_{n-1}\right)+k_{2}\left(\rho_{n}, \tau_{n-1}\right)\right] \phi_{0}\left(\tau_{n-1}\right)\left[\frac{d \tau^{-}(\sigma)}{d \sigma}\right]_{\sigma=\rho_{n}}^{-1} .
\end{gathered}
$$

By using (3.16), (3.17), (3.18) and (3.20), it follows for $n \rightarrow \infty$ that

$$
\frac{\left[R^{-}\right]^{2}}{1-R^{-}} \tau_{n} \phi_{0}\left(\tau_{n}\right)-\frac{\left[R^{+}\right]^{2}}{1-R^{+}} \tau_{n-1} \phi_{0}\left(\tau_{n-1}\right) \rightarrow 0
$$

so that by using, cf. (7.6), $\tau_{n+1} / \tau_{n} \rightarrow \lambda^{-1}=R^{+} / R^{-}$, it is clear that

$$
\frac{\phi_{0}\left(\tau_{n}\right)}{\phi_{0}\left(\tau_{n-1}\right)} \rightarrow \frac{R^{+}}{R^{-}} \frac{1-R^{-}}{1-R^{+}}<0 \text {. }
$$

Subtraction of (9.14) (i) and (ii), and also (9.14) (iii) and (iv) yields, after elimination of $\omega_{2}\left(\rho_{n}\right)-\omega_{1}\left(\rho_{n}\right)$,

$$
\begin{gathered}
\frac{2 a \tau_{n}}{\rho_{n}-\tau_{n}}\left[\frac{d \tau^{+}(\sigma)}{d \sigma}\right]_{\sigma=\rho_{n}}^{-1} b\left(\tau_{n}\right)+\frac{2 a \tau_{n-1}}{\rho_{n}-\tau_{n-1}}\left[\frac{d \tau^{-}(\sigma)}{d \sigma}\right]_{\sigma=\rho_{n}}^{-1} b\left(\tau_{n-1}\right) \\
=\frac{1}{\tau_{n}}\left[-k_{1}\left(\rho_{n}, \tau_{n}\right)+k_{2}\left(\rho_{n}, \tau_{n}\right)\right] \tau_{n} \phi_{0}\left(\tau_{n}\right)\left[\frac{d \tau^{+}(\sigma)}{d \sigma}\right]_{\sigma=\rho_{n}}^{-1} \\
+\frac{1}{\tau_{n-1}}\left[-k_{1}\left(\rho_{n}, \tau_{n-1}\right)+k_{2}\left(\rho_{n}, \tau_{n-1}\right)\right] \tau_{n-1} \phi_{0}\left(\tau_{n-1}\right)\left[\frac{d \tau^{-}(\sigma)}{d \sigma}\right]_{\sigma=\rho_{n}}^{-1}
\end{gathered}
$$

Again, by using (3.16), (3.17), (3.18), and (3.20), it follows for $n \rightarrow \infty$ :

$$
\frac{2 a R^{-}}{R^{-}-1} b\left(\tau_{n}\right)+\frac{2 a R^{+}}{R^{+}-1} b\left(\tau_{n-1}\right)
$$




$$
=-a\left(\pi_{2}-\pi_{1}\right)\left\{\frac{\left[R^{-}\right]^{2}}{1-R^{-}} \tau_{n} \phi_{0}\left(\tau_{n}\right)-\frac{\left[R^{+}\right]^{2}}{1-R^{+}} \tau_{n-1} \phi_{0}\left(\tau_{n-1}\right)\right\} \rightarrow 0 .
$$

Hence,

$$
\frac{b\left(\tau_{n}\right)}{b\left(\tau_{n-1}\right)} \rightarrow \frac{R^{+}}{R^{-}} \frac{1-R^{-}}{1-R^{+}}
$$

From (9.14) (i), with $n$ replaced by $m+n$, it follows, for $m$ sufficiently large again by using (3.17), (3.18), (3.20) and the asymptotic relations for $b\left(\tau_{m}\right)$ and $\phi_{0}\left(\tau_{m}\right)$ obtained above that for $n=1,2, \ldots$,

$$
\begin{gathered}
\omega_{2}\left(\rho_{m+n}\right)=-\frac{a R^{-}}{R^{-}-1}\left\{b\left(\tau_{m}\right)\left[\frac{R^{+}}{R_{-}} \frac{R^{-}-1}{R^{+}-1}\right]^{n}\right. \\
\left.+a \pi_{2} R^{-} \tau_{m} \phi_{0}\left(\tau_{m}\right)\left[\frac{R^{+}}{R^{-}} \frac{1-R^{-}}{1-R^{+}}\right]^{n}\right\}
\end{gathered}
$$

which yields that

$$
\frac{\omega_{2}\left(\rho_{n+1}\right)}{\omega_{2}\left(\rho_{n}\right)} \rightarrow \frac{R^{+}}{R^{-}} \frac{1-R^{-}}{1-R^{+}} \text {. }
$$

Hence (9.15) is proved.

As in (8.2), we introduce for the present case the meromorphic functions

$$
\begin{gathered}
\widetilde{B}(\tau):=\sum_{n=0}^{\infty} \frac{b\left(\tau_{n}\right)}{\tau-\tau_{n}}\left(\frac{\tau}{\tau_{n}}\right)^{m_{b}} \\
\widetilde{\Phi}_{0}(\tau):=\sum_{n=0}^{\infty} \frac{\phi_{0}\left(\tau_{n}\right)}{\tau-\tau_{n}}\left(\frac{\tau}{\tau_{n}}\right)^{m_{\phi}}, \\
\widetilde{\Omega}_{i}(\tau):=\sum_{n=1}^{\infty} \frac{\omega_{i}\left(\rho_{n}\right)}{\rho-\rho_{n}}\left(\frac{\rho}{\rho_{n}}\right)^{m_{i}}, \\
\tau_{0}=T, \rho_{n+1}=\rho^{+}\left(\tau_{n}\right), \tau_{n}=\tau^{+}\left(\rho_{n}\right),
\end{gathered}
$$

and

$$
m_{b} \geq \tilde{M}, m_{\phi} \geq \tilde{M}, m_{i} \geq \tilde{M}, i=1,2 ;
$$

here $\tilde{M}$ is uniquely defined as the nonnegative integer such that (note $0<\lambda<1$ ).

(i) $\quad \tilde{M}=0$, for $|\psi \lambda|<1$,

(ii) $\quad\left|\psi \lambda^{\tilde{M}+1}\right|<1 \leq\left|\psi \lambda^{\tilde{M}}\right|$ otherwise.

By using (7.6) and Lemma 9.1, it is readily seen, since for $k$ large,

$$
\frac{b\left(\tau_{n+k}\right)}{\left[\tau_{n+k}\right]^{m+1}} \sim \frac{b\left(\tau_{k}\right)}{\left[\tau_{k}\right]^{m+1}}\left(\psi \lambda^{m+1}\right)^{n}
$$

that for $m \geq \widetilde{M}$ the function $\widetilde{B}(\tau)$ is a well-defined meromorphic function which is regular in $|\tau| \leq 1$. Similar statements are true for the other functions defined in (9.19).

$$
\text { Because, cf. (9.15), } \quad \lambda \psi=\frac{R^{-}-1}{R^{+}-1},
$$

and since it is readily verified by using (2.3) and (3.16) that $|\lambda \psi|<1$, we take, from now on in (9.19),

$$
m=\tilde{M}=0 .
$$


Theorem 9.1: For $a_{1}=a_{2}=a<2, \pi_{1} \neq \pi_{2} \neq 1$, the functions $B(\cdot), \Phi_{0}(\cdot)$, $\Omega_{i}(\cdot), 1,2$, which satisfy conditions $(2.10)$ are given by

(i) $\quad B(s)=-\widetilde{B}(0)+\widetilde{B}(s)$,

(ii) $\Phi_{0}(s)=\widetilde{\Phi}(s)$,

(iii) $\quad \Omega_{i}(s)=\widetilde{\Phi}(0)-\widetilde{\Omega}_{i}(0)+\widetilde{\Omega}_{i}(s), i=1,2$;

here $\widetilde{B}(\cdot), \widetilde{\Phi}_{0}(\cdot), \widetilde{\Omega}_{i}(\cdot), i=1,2$, are given by $(9.19)$ with $m_{b}=m_{\phi}=m_{2}=m_{1}=0$, and they all contain $\phi_{0}(T)$ as a factor, which is determined by

$$
\Omega_{2}(1)+\Omega_{1}(1)=2-a .
$$

If $a \geq 2$ no stationary joint distribution exists.

Proof: As in Section 8, introduce the polynomials $\widehat{B}(\cdot), \widehat{\Phi}_{0}(\cdot), \widehat{\Omega}_{i}(\cdot), i=1,2$. The degrees of these functions are determined by the same arguments as used in Section 8 for the case $\widetilde{M}=1$; then the proof is rendered as in Theorem 8.1 and is therefore omitted here. Note that for the present case, $\widehat{\mu}_{i}=0, i=1,2 ; \mathrm{cf}$. (8.12).

Theorem 9.1 provides all results needed to get the characteristics of the queue length $\left(\mathbf{x}_{1}, \mathbf{x}_{2}\right)$ stationary joint distribution. The following analysis provides some detailed information about the influence of the probabilities $\pi_{i}, i=1,2, \pi_{1}+\pi_{2}=1$; cf. (2.3).

Set

$$
\Omega(r):=\frac{1}{2}\left\{\Omega_{1}(r)+\Omega_{2}(r)\right\}
$$

Elimination of $B(\cdot)$ from (4.3) and (4.4) yields for the present case, i.e., $a_{1}=a_{2}=a$, $\pi_{1} \neq \pi_{2}$,

$$
\Omega\left(r^{ \pm}(t)\right)+\left[-1+\frac{1}{2} a^{2} t-\frac{1}{2} a r^{ \pm}(t)\right] \Phi_{0}(t)=0,
$$

with $\left(r^{ \pm}(t), t\right)$ a zero-tuple of

$$
h(r, t) \equiv a t^{2}+[1-(2+a) r] t+r^{2}=0 .
$$

Relations (9.25), (9.26) formulate a functional equation which is identical to that of the symmetrical shortest queue; cf. (3.6) of [12]. Hence, the solution constructed in [12] can be used here.

Put, cf. (3.2), (3.3) and also [12],

(i) $\quad r_{n+1}^{+}:=r^{+}\left(t_{n}^{+}\right), t_{n}^{+}:=t^{+}\left(r_{n}^{+}\right), n=0,1, \ldots$ with

$$
t_{0}^{+}=\frac{4}{a^{2}}, r_{0}^{+}=\frac{2}{a}
$$

with

(ii) $\quad t_{n+1}^{-}=t^{+}\left(r_{n}^{-}\right), r_{n}^{-}=r^{+}\left(t_{n}^{-}\right), n=0,1, \ldots$,

$$
t_{0}^{-}=-\frac{1}{a}, r_{0}^{-}=-1-\frac{2}{a} \text {. }
$$

The solution of (9.25) and (9.26) is then expressed by, cf. (4.7) of [12],

$$
\Phi_{0}(t)=\Phi(1) \frac{\prod_{n=1}^{\infty}\left(1-\frac{t}{t_{n}^{-}}\right)}{\prod_{n=1}^{\infty}\left(1-\frac{1}{t_{n}^{-}}\right)} \frac{\prod_{n=0}^{\infty}\left(1-\frac{1}{t_{n}^{+}}\right)}{\prod_{n=0}^{\infty}\left(1-\frac{t}{t_{n}^{+}}\right)}
$$


with

$$
\Omega(r)=\Omega(1) \frac{\prod_{n=0}^{\infty}\left(1-\frac{r}{r_{n}^{-}}\right)}{\prod_{n=0}^{\infty}\left(1-\frac{1}{r_{n}^{-}}\right)} \frac{\prod_{n=1}^{\infty}\left(1-\frac{1}{r_{n}^{+}}\right)}{\prod_{n=1}^{\infty}\left(1-\frac{r}{r_{n}^{+}}\right)}
$$

$$
\Omega(1)=\frac{1}{2}(2-a), \Phi_{0}(1)=\frac{1}{1+a} .
$$

Here the first relation in (9.29) follows from (2.5) and (9.1) and the second one is obtained from (9.25) for the zero-tuple $(r, t)=(1,1)$. Because the zero-tuples in $(9.27)$ are independent of $\pi_{i}, i=1,2$, it is seen that

$$
\Phi_{0}(t) \text { and } \Omega(r) \text { are independent of } \pi_{i}, i=1,2 .
$$

Consequently, the stationary distribution of $\mathbf{x}_{1}+\mathbf{x}_{2}$, that of $\max \left\{\mathbf{x}_{1}, \mathbf{x}_{2}\right\}$ and of $\min \left(\mathbf{x}_{1}, \mathbf{x}_{2}\right)$ are all independent of $\pi_{i}, i=1,2$, cf. [2].

\section{Some Expressions for Probabilities and Moments, $a_{1} \neq a_{2}$}

In this section, we derive some expressions for several characteristics of the queue lengths.

We consider first the case

$$
a_{1} \neq 1, a_{2} \neq 1, a_{1} \neq a_{2}, \frac{1}{a_{1}}+\frac{1}{a_{2}}>1,
$$

since we have to discuss separately the case that one of the $a_{i}$ 's is equal to one.

From(2.4) and Appendix D, we have

$$
\begin{gathered}
\mathrm{E}\left\{r^{\mathbf{x}_{1}}\left(\mathbf{x}_{2}>\mathbf{x}_{1}\right)\right\}=\frac{1}{1-a_{1} r} \Omega_{2}(1)-\frac{1}{1-a_{1} r} \Phi_{0}(r)-\frac{a_{1} r}{1-a_{1} r} \frac{B(r)}{r-1} \\
\mathrm{E}\left\{r^{\mathbf{x}_{2}}\left(\mathbf{x}_{2}>\mathbf{x}_{1}\right)\right\}=a_{2} r \pi_{2} \Phi_{0}(r)-a_{2} r \frac{B(r)}{r-1}
\end{gathered}
$$

The latter yields, cf. (d.4),

(i) $\quad \mathrm{E}\left\{\left(\mathbf{x}_{2}>\mathrm{x}_{1}\right)\right\}=\frac{1}{1-a_{1}} \Omega_{2}(1)-\frac{1}{1-a_{1}} \Phi_{0}(1)-\left.\frac{a_{1}}{1-a_{1}} \frac{d}{d r} B(r)\right|_{r=1}$,

(ii) $\mathrm{E}\left\{\left(\mathbf{x}_{2}>\mathrm{x}_{1}\right)\right\}=a_{2} \pi_{2} \Phi_{0}(1)-\left.a_{2} \frac{d}{d r} B(r)\right|_{r=1}$,

(iii) $\mathrm{E}\left\{\left(\mathrm{x}_{1}=\mathrm{x}_{2}\right)\right\}=\Phi_{0}(1)$,

(iv) $\quad \mathrm{E}\left\{\left(\mathbf{x}_{1}>\mathbf{x}_{2}\right)\right\}=a_{1} \pi_{1} \Phi_{0}(1)+\left.a_{1} \frac{d}{d r} B(r)\right|_{r=1}$

here (10.3) (iv) is obtained from (10.3) (ii) by interchanging $a_{1}$ and $a_{2}$ and by changing the sign of the term with $B(\cdot)$, cf. $(2.4)(i)$ and (2.4) $(i i)$.

It follows from (10.3) $(i i)-(i v)$, that

$$
1=\left\{1+a_{2} \pi_{2}+a_{1} \pi_{1}\right\} \Phi_{0}(1)+\left.\left(a_{1}-a_{2}\right) \frac{d}{d r} B(r)\right|_{r=1}
$$

so that

$$
\begin{gathered}
\left.\Phi_{0}(1)=\left.\frac{1}{1+a_{1} \pi_{1}+a_{2} \pi_{2}}\left\{1-\left(a_{1}-a_{2}\right) \frac{d}{d r} B(r)\right\}\right|_{r=1}\right\} \\
\mathrm{E}\left\{\left(\mathbf{x}_{2}>\mathbf{x}_{1}\right)\right\}=\frac{a_{2}}{a_{1}-a_{2}}\left[-1+\left(1+a_{1}\right) \Phi_{0}(1)\right],
\end{gathered}
$$




$$
\mathrm{E}\left\{\left(\mathbf{x}_{1}>\mathbf{x}_{2}\right)\right\}=\frac{a_{1}}{a_{2}-a_{1}}\left[-1+\left(1+a_{2}\right) \Phi_{0}(1)\right] .
$$

From (d.6) and (d.4), we obtain for the present case, cf. (10.1),

(i) $\quad \mathrm{E}\left(\mathbf{x}_{2}\left(\mathbf{x}_{2}>\mathbf{x}_{1}\right)\right\}=a_{2} \pi_{2} \Phi_{0}(1)+\left.a_{2} \pi_{2} \frac{d}{d r} \Phi_{0}(r)\right|_{r=1}$

$$
-\left.a_{2} \frac{d}{d r} B(r)\right|_{r=1}-\left.\frac{1}{2} a_{2} \frac{d^{2}}{d r^{2}} B(r)\right|_{r=1}
$$

(ii) $\mathrm{E}\left\{\mathbf{x}_{1}\left(\mathbf{x}_{1}>\mathbf{x}_{2}\right\}=a_{1} \pi_{1} \Phi_{0}(1)+\left.a_{1} \pi_{1} \frac{d}{d r} \Phi_{0}(r)\right|_{r=1}\right.$

$$
+\left.a_{1} \frac{d}{d r} B(r)\right|_{r=1}+\left.\frac{1}{2} a_{1} \frac{d^{2}}{d r^{2}} B(r)\right|_{r=1}
$$

(iii) $\mathrm{E}\left\{\mathbf{x}_{1}\left(\mathbf{x}_{1}=\mathbf{x}_{2}\right)\right\}=\left.\frac{d}{d r} \Phi_{0}(r)\right|_{r=1}$,

(iv) $\quad \mathrm{E}\left\{\mathbf{x}_{1}\left(\mathbf{x}_{2}>\mathbf{x}_{1}\right)\right\}=\frac{a_{1}}{\left(1-a_{1}\right)^{2}}\left\{\Omega_{2}(1)-\Phi_{0}(1)-\left.\frac{d}{d r} B(r)\right|_{r=1}\right\}$

$$
-\left.\frac{1}{1-a_{1}} \frac{d}{d r} \Phi_{0}(r)\right|_{r=1}-\left.\frac{1}{2} \frac{a_{1}}{1-a_{1}} \frac{d^{2}}{d r^{2}} B(r)\right|_{r=1} .
$$

The summation of (10.5), (ii)-(iv) yields the expression for $\mathrm{E}\left\{\mathbf{x}_{1}\right\}$. The expression for $\mathrm{E}\left\{\mathbf{x}_{2}\right\}$ then follows by interchanging $a_{1}$ and $a_{2}$ and changing the signs of the terms containing $B(\cdot)$.

Next, we consider the case

$$
a_{1}=1, a_{2} \neq 1, \frac{1}{a_{1}}+\frac{1}{a_{2}}>1
$$

By noting that relations (10.3) (ii)-(iv) and (10.4) have been all derived from (d.6), in which $1-a_{1}$ does not occur, it is seen that these relations also apply to the present case with $a_{1}=1$.

From (d.10), we obtain for the present case (10.6):

(i) $\quad \mathrm{E}\left\{\mathbf{x}_{1}\left(\mathbf{x}_{2}>\mathbf{x}_{1}\right)\right\}=\left.\frac{1}{2} \frac{d^{2}}{d r^{2}} \Phi_{0}(r)\right|_{r=1}+\left.\frac{1}{2} \frac{d^{2}}{d r^{2}} B(r)\right|_{r=1}$,

(ii) $\mathrm{E}\left\{\mathbf{x}_{1}\left(\mathbf{x}_{1}=\mathbf{x}_{2}\right)\right\}=\left.\frac{d}{d r} \Phi_{0}(r)\right|_{r=1}$,

(iii) $\mathrm{E}\left\{\mathbf{x}_{1}\left(\mathbf{x}_{1}>\mathbf{x}_{2}\right)\right\}=\pi_{1} \Phi_{0}(1)+\left.\pi_{1} \frac{d}{d r} \Phi_{0}(r)\right|_{r=1}+\left.\frac{d}{d r} B(r)\right|_{r=1}$

$$
+\left.\frac{1}{2} \frac{d^{2}}{d r^{2}} B(r)\right|_{r=1}
$$

Here (10.7) (iii) follows from (10.5) (ii) with $a_{1}=1$; note that (10.5) (ii) has been derived from (d.6).

\section{Appendix A}

Let $(\xi, \eta)$ be two stochastic variables with a joint distribution given by

$$
\begin{gathered}
\operatorname{Pr}\{\boldsymbol{\xi}=2, \boldsymbol{\eta}=0\}=\frac{a_{1} a_{2}}{b}, \\
\operatorname{Pr}\{\boldsymbol{\xi}=1, \boldsymbol{\eta}=0\}=\frac{a_{1}}{b},
\end{gathered}
$$




$$
\operatorname{Pr}\{\xi=0, \boldsymbol{\eta}=2\}=\frac{a_{2}}{b}
$$

Hence, we have, from (2.8),

$$
h_{1}(\rho, \tau)=0 \Leftrightarrow E\left\{\tau^{\xi} \rho^{\eta}\right\}=\rho \tau .
$$

Put $\tau=p \rho$ in (a.2), so that

$$
h_{1}(\rho, p \rho)=0 \Leftrightarrow p=\mathrm{E}\left\{p^{\boldsymbol{\xi}} \rho^{\boldsymbol{\xi}}+\boldsymbol{\eta}-2\right\} .
$$

Note that (a.1) implies $\operatorname{Pr}\{\xi+\eta-2 \leq 0\}=1$, so that for $|\rho| \geq 1, \rho \neq 1$ and $|p|=1$,

$$
\left|\mathrm{E}\left\{p^{\boldsymbol{\xi}} \rho^{\boldsymbol{\xi}+\boldsymbol{\eta}-2}\right\}\right| \leq \mathrm{E}\left\{|\rho|^{\boldsymbol{\xi}}+\boldsymbol{\eta}-2\right\}<1=|p| .
$$

For fixed $\rho,|\rho| \geq 1$, the last term in (a.3) is regular for $|p|<1$ and continuous for $|p| \leq 1$. It follows by applying Rouché's theorem that $h_{1}(\rho, p \rho)$ has for $|\rho| \geq 1, \rho \neq 1$ exactly one zero in $|p|<1$, and so the other zero of $h_{1}(\rho, p \rho)$ lies in $|p|>1$; cf. (a.4). Note that $h_{1}(\rho, p \rho)$ is a quadratic function in $p$. This proves the first statement in Lemma 3.1, the proof of the other ones is similar.

\section{Appendix B}

In this appendix, we prove Lemma 4.1 and relations (4.2). From (2.9), for zero-tuple $|\rho|=1, \tau=\tau^{-}(\rho)$ (note $\left|\tau^{-}(\rho)\right| \leq|\rho|=1$, cf. (3.2)), we have

$$
-\Omega_{2}(\rho)=\frac{a_{1} \tau^{-}(\rho)}{\rho-\tau^{-}(\rho)} B\left(\tau^{-}(\rho)\right)+k_{1}\left(\rho, \tau^{-}(\rho)\right) \Phi_{0}\left(\tau^{-}(\rho)\right),|\rho|=1
$$

The function $\Omega_{2}(\rho)$ is regular in $|\rho|<1$ and continuous in $|\rho| \leq 1$. Consequently, the right-hand side of (b.1) can be continued analytically into $|\rho|<1$. Consider this analytic continuation of the right-hand side of (b.1) along a simple contour in $|\rho| \leq 1$, starting at a point $\sigma_{0}$ with $\left|\sigma_{0}\right|=1, \sigma_{0} \neq 1$ and such that it intersects the interval $\left[\rho^{-}, \rho^{+}\right]$, cf. (3.4), only once at an interior point $\sigma_{1}$, say, with $\rho^{-}<\sigma_{1}<\rho^{+}$. Because $\tau^{-}\left(\sigma_{1}\right)=\overline{\tau^{+}\left(\sigma_{1}\right)}$, it is seen that the analytic continuation of the right-hand side along this simple contour leads, on its return to $\sigma_{0}$, to

$$
-\Omega_{2}\left(\sigma_{0}\right)=\frac{a_{1} \tau^{+}\left(\sigma_{0}\right)}{\rho-\tau^{+}\left(\sigma_{0}\right)} B\left(\tau^{+}\left(\sigma_{0}\right)\right)+k_{1}\left(\sigma_{0}, \tau^{+}\left(\sigma_{0}\right)\right) \Phi_{0}\left(\tau^{+}\left(\sigma_{0}\right)\right) .
$$

This relation holds for all $\left|\sigma_{0}\right|=1, \sigma_{0} \neq 1$, and so, by continuity it also holds for $\sigma_{0}=1$.

Hence, we obtain the following set of relations, of which the last three are motivated by analogous arguments to those used in deriving the first one:

$$
\begin{gathered}
\Omega_{2}(\rho)+\frac{a_{1} \tau^{ \pm}(\rho)}{\rho-\tau^{ \pm}(\rho)} B\left(\tau^{ \pm}(\rho)\right)+k_{1}\left(\rho, \tau^{ \pm}(\rho)\right) \Phi_{0}\left(\tau^{ \pm}(\rho)\right)=0,|\rho|=1, \\
\Omega_{1}(r)-\frac{a_{2} t^{ \pm}(r)}{r-t^{ \pm}(r)} B\left(t^{ \pm}(r)\right)+k_{2}\left(r, t^{ \pm}(r)\right) \Phi_{0}\left(t^{ \pm}(r)\right)=0,|r|=1, \\
\Omega_{2}\left(\rho^{ \pm}(\tau)\right)+\frac{a_{1} \tau}{\rho^{ \pm}(\tau)-\tau} B(\tau)+k_{1}\left(\rho^{ \pm}(\tau), \tau\right) \Phi_{0}(\tau)=0,|\tau|=1,
\end{gathered}
$$




$$
\Omega_{1}\left(r^{ \pm}(t)\right)-\frac{a_{2} t}{r^{ \pm}(t)-t} B(t)+k_{2}\left(r^{ \pm}(t), t\right) \Phi_{0}(t)=0,|t|=1
$$

with

$$
\begin{gathered}
\left(\rho, \tau^{ \pm}(\rho)\right) \text { and }\left(\rho^{ \pm}(\tau), \tau\right) \text { zero-tuples of } h_{1}(\rho, \tau), \\
\left(r, t^{ \pm}(r)\right) \text { and }\left(r^{ \pm}(t), t\right) \text { zero-tuples of } h_{2}(r, t) .
\end{gathered}
$$

Because, cf. (3.2), $\left|\tau^{+}(\rho)\right|>1$ for $|\rho|=1, \rho \neq 1$, it is seen from (b.3) that there is a domain in $|\tau|>1$ where $\Phi_{0}(\tau)$, and similarly, $B(\tau)$ is regular; note that $k_{1}(\rho, \tau)$ is regular in $|\rho| \geq 1,|\tau| \geq 1$. Also $\tau^{+}(\rho)$ is regular in $|\rho| \geq 1$, with $|\rho|$ and $|\tau|$ finite. Further, it is seen, that in $|\rho| \geq 1$ domains exist where $\Omega_{2}(\rho)$ and $\Omega_{1}(\rho)$ are regular. Next, take $\tau$ in the domain where $\Phi_{0}(\tau)$ and $B(t)$ are regular, i.e., in the domain defined by $\left\{\tau: \tau=\tau^{+}(\rho),|\rho|=1\right\}$. For such $\tau$ it is seen from (b.5) that $\Omega_{2}\left(\rho^{+}(\tau)\right)$ is regular. Since $\left|\rho^{+}(\tau)\right|>|\tau|, \tau \neq 1$, it follows that the domain outside $|\rho|=1$, where $\Omega_{2}(\rho)$ is regular, can be again extended. So, by repeatedly using relations (b.3) and (b.5) the domains of regularity of $\Phi_{0}(\tau), B_{0}(\tau)$ and $\Omega_{2}(\rho)$ can be recursively extended; analogously for $\Omega_{1}(r)$. Because $\left|\rho^{+}(\tau)\right|>|\tau|$ in $|\tau|>1,\left|\tau^{+}(\rho)\right|>|\rho|$ in $|\rho|>1$, it follows that the domain in $|\tau|>1$ where $\Phi_{0}(\tau)$ is regular is unbounded, similarly for $B(\tau)$, and analogously for $\Omega_{2}(\rho)$ and $\Omega_{1}(r)$.

The singularities of $\Phi_{0}(\tau)$ in $|\tau|>1$ can only be poles, because $k_{1}\left(\rho, \tau^{ \pm}(\rho)\right)$ and $k_{2}\left(\rho^{ \pm}(\tau), \tau\right)$ are regular in $|\rho|>1$ and $|\tau|>1$, respectively; note (3.4), and similarly for the other coefficients in (b.3)-(b.6). Further $\Phi_{0}(\tau)$ has at least one pole in $\{\tau: 1<|\tau| \leq \infty\}$, because if $\Phi_{0}(\tau)$ would be regular here, then, since it is also regular for $|\tau|<1$, cf. (2.10), it is necessarily a constant, as Liouville's theorem implies. Analogously, for $B(\tau), \Omega_{2}(\rho)$ and $\Omega_{1}(r)$. Consequently, Lemma 4.1 is proved. $\square$

From the analytic continuations discussed above, it is seen that the relations (b.3)(b.6) hold for all those $\tau, t, \rho$ and $r$ where the functions in (b.3)-(b.6) are finite. Consequently, it is seen that the validity of the relations (4.1)-(4.4) has been established.

\section{Appendix C}

The integer $\tilde{M} \geq 0$ has been defined in (8.6). Numerical results indicate that $\tilde{M}$ is always larger than zero. A proof of $\tilde{M}>0$ seems to be rather lengthy and intricate. Below we discuss some cases, for which the proof is fairly simple.

First consider

$$
a_{1}=a_{2}=a<2, \pi_{1}=\pi_{2}=\frac{1}{2}
$$

From (c.1), (3.17) and (7.7) (iv) it follows that

$$
\lambda_{1} \mu_{1}=\frac{R_{1}^{-}-1}{R_{1}^{+}-1} \frac{R_{1}^{-}+R_{1}^{+}}{2 R_{1}^{-}}=\frac{R_{1}^{-}-1}{R_{1}^{+}-1} \frac{b / a}{2 R_{1}^{-}} .
$$

From (3.16), (3.20), (c.1) and (c.2) it results that

$$
\begin{gathered}
-2 \lambda_{1} \mu_{2}=(2+a)\left(R_{1}^{+}-1\right)\left(a-R_{1}^{-}\right)=\frac{2+a}{2-a-(1-a) R_{1}^{+}} \\
R_{1}^{+}=\frac{1}{2}\left(2+a+\sqrt{4+a^{2}}\right) .
\end{gathered}
$$

From (c.3), it is not difficult to verify, since $\lambda_{1} \mu_{1}=\lambda_{2} \mu_{2}$ for the present case, that 


$$
\left|\lambda_{1} \mu_{1}+\lambda_{2} \mu_{2}\right|>1 \text { for } 0<a<2
$$

so that for the present case $\tilde{M}>0$.

Because $\lambda_{1} \mu_{1}$ is a continuous function in each of the parameters $a_{1}, a_{2}, \pi_{1}=1-$ $\pi_{2}$, it follows from the results so far obtained that $\tilde{M}$ is larger than zero for $\mid a_{1}$ $a_{2}\left|<\epsilon_{1},\right| \pi_{1}-\frac{1}{2} \mid<\epsilon_{2}, 1 / a_{1}+1 / a_{2}>1$, with $\epsilon_{1}$ and $\epsilon_{2}$ sufficiently small.

Next we consider the case

$$
a_{1}>1, \pi_{1}=\pi_{2}=\frac{1}{2} \text {. }
$$

From (3.17), (7.7) (iv) and (c.4) we have

$$
\begin{gathered}
\lambda_{1} \mu_{1}=\frac{\left(R_{1}^{-}-1\right)\left(R_{2}^{-}+R_{1}^{+}\right)}{\left(R_{1}^{+}-1\right)\left(R_{2}^{-}+R_{1}^{-}\right)}=\frac{R_{1}^{-} R_{2}^{-}+\frac{1}{a_{1}}-R_{2}^{-}-R_{1}^{+}}{R_{1}^{+} R_{2}^{-}+\frac{1}{a_{1}}-R_{2}^{-}-R_{1}^{-}} \\
=-1+\frac{R_{2}^{-}\left(R_{1}^{+}+R_{1}^{-}\right)+\frac{2}{a_{1}}-2 R_{2}^{-}-\left(R_{1}^{+}+R_{1}^{-}\right)}{R_{1}^{+} R_{2}^{-}+\frac{1}{a_{1}}-R_{2}^{-}-R_{1}^{-}} \\
=-1+\frac{\left(R_{2}^{-}-1\right)\left(\frac{b}{a_{2}}-2\right)+2\left(\frac{1}{a_{1}}-1\right)}{R_{1}^{+} R_{2}^{-}+\frac{1}{a_{1}}-2 R_{2}^{-}} .
\end{gathered}
$$

Because $R_{1}^{+}-1>0$, the denominators in (c.5) are positive and the numerator in the last term of (c.5) is not positive for $a_{1} \geq 1$, noticing that

$$
\frac{b}{a_{2}}-2=-1+a_{1}+\frac{a_{1}}{a_{2}}>0 \text { for } a_{1} \geq 1
$$

it follows that $\lambda_{1} \mu_{1}<-1$. Hence, from $\lambda_{2} \mu_{2}<0$ we obtain $\left|\lambda_{1} \mu_{1}+\lambda_{2} \mu_{2}\right|>1$, i.e., $\widetilde{M}>0$. Analogously, $\tilde{M}>0$ for $a_{2} \geq 1, \pi_{1}=\pi_{2}=1 / 2$.

Finally, consider the case $\pi_{1}=\pi_{2}=\frac{1}{2}$ and $a_{2} \downarrow 0$. It is readily verified that $R_{2}^{-} \rightarrow 0$ for $a_{2} \downarrow 0$ and so it is seen from (c.5) that for $a_{2}$ sufficient small, the numerator in the last term of (c.5) will be negative and it follows again that $\lambda_{1} \mu_{1}<-1$ and so $\tilde{M}>0$, since $\lambda_{2} \mu_{2}<0$.

\section{Appendix D}

From (2.4), it is seen that for $|r| \leq 1$,

$$
\begin{gathered}
\mathrm{E}\left\{r^{\mathbf{x}_{1}}\left(\mathbf{x}_{2}>\mathbf{x}_{1}\right)\right\} \frac{1-a_{1} r}{a_{1} r}(1-r)-\frac{1-r}{a_{1} r} \Omega_{2}(1)+\frac{1-r}{a_{1} r} \Phi_{0}(r)-B(r)=0, \\
\mathrm{E}\left\{r^{\mathbf{x}_{2}}\left(\mathbf{x}_{2}>\mathbf{x}_{1}\right)\right\} \frac{1-r}{a_{2} r}-(1-r) \pi_{2} \Phi_{0}(r)-B(r)=0 .
\end{gathered}
$$

So that by letting $r \rightarrow 1$, it follows, cf. (2.10), that

$$
B(1)=0 \text {. }
$$

Because the smallest pole of $B(\tau)$ as well as of $\Phi_{0}(\tau)$ is $\tau=\tau_{0}^{(0)}=T>1$, cf. Lemma $5.1,(8.2)$ and (8.7), it follows that there exists a neighborhood of $\tau=1$ where $B(\cdot)$ and $\Phi_{0}(\cdot)$ are both regular, so that all derivatives of $B(\cdot)$ and $\Phi_{0}(\cdot)$ at $r=1$ are 
finite. Thus we may write, (cf. (d.3)): for $r \sim 1$,

$$
\begin{gathered}
B(r)=\left.(r-1) \frac{d}{d r} B(r)\right|_{r=1}+\left.\frac{1}{2}(r-1)^{2} \frac{d}{d r^{2}} B(r)\right|_{r=1} \\
+\left.\frac{1}{6}(r-1)^{3} \frac{d^{3}}{d r^{3}} B(r)\right|_{r=1}+O\left((r-1)^{4}\right), \\
\Phi_{0}(r)=\Phi_{0}(1)+\left.(r-1) \frac{d}{d r} \Phi_{0}(r)\right|_{r=1}+\left.\frac{1}{2}(r-1)^{2} \frac{d^{2}}{d r^{2}} \Phi_{0}(r)\right|_{r=1}+O\left((r-1)^{3}\right) .
\end{gathered}
$$

From (d.1), we have: for $r \sim 1$,

$$
\begin{gathered}
\mathrm{E}\left\{r^{\left.\mathbf{x}_{1}\left(\mathbf{x}_{2}>\mathbf{x}_{1}\right)\right\}}=\frac{1}{1-a_{1} r} \Omega_{2}(1)-\frac{1}{1-a_{1} r}\left[\Phi_{0}(1)+\left.(r-1) \frac{d}{d r} \Phi_{0}(r)\right|_{r=1}\right.\right. \\
\left.+\left.\frac{1}{2}(r-1)^{2} \frac{d^{2}}{d r^{2}} \Phi_{0}(r)\right|_{r=1}+\left.\frac{1}{6}(r-1)^{3} \frac{d^{3}}{d r^{3}} \Phi_{0}(r)\right|_{r=1}+O\left((r-1)^{4}\right)\right] \\
-\frac{a_{1} r}{1-a_{1} r}\left[\left.\frac{d}{d r} B(r)\right|_{r=1}+\left.\frac{1}{2}(r-1) \frac{d^{2}}{d r^{2}} B(r)\right|_{r=1}\right. \\
\left.+\left.\frac{1}{6}(r-1)^{2} \frac{d^{3}}{d r^{3}} B(r)\right|_{r=1}+O\left((r-1)^{3}\right)\right]
\end{gathered}
$$

and from (d.2),

$$
\mathrm{E}\left\{r^{\mathbf{x}_{2}}\left(\mathbf{x}_{2}>\mathbf{x}_{1}\right)\right\}=a_{2} \pi_{2} r \Phi_{0}(r)-a_{2} r \frac{B(r)}{r-1)} .
$$

For, cf. Theorem 8.1,

$$
a_{1}=1, a_{2} \neq 1, \frac{1}{a_{1}}+\frac{1}{a_{2}}>1,
$$

we have from (d.5): for $r \sim 1$,

$$
\begin{gathered}
\mathrm{E}\left\{r^{\mathbf{x}_{1}}\left(\mathbf{x}_{2}>\mathbf{x}_{1}\right)\right\}=\frac{1}{1-r}\left[\Omega_{2}(1)-\Phi_{0}(1)-\left.(r-1) \frac{d}{d r} \Phi_{0}(r)\right|_{r=1}\right. \\
-\left.\frac{1}{2}(r-1)^{2} \frac{d^{2}}{d r^{2}} \Phi_{0}(r)\right|_{r=1}-\left.\frac{1}{6}(r-1)^{3} \frac{d^{3}}{d r^{3}} \Phi_{0}(r)\right|_{r=1}+O\left((1-r)^{4}\right) \\
-\frac{r}{1-r}\left[\left.\frac{d}{d r} B(r)\right|_{r=1}+\left.\frac{1}{2}(r-1) \frac{d^{2}}{d r^{2}} B(r)\right|_{r=1}\right. \\
\left.+\left.\frac{1}{6}(r-1)^{2} \frac{d^{3}}{d r^{3}} B(r)\right|_{r=1}+O\left((1-r)^{3}\right)\right] .
\end{gathered}
$$

From (d.7) and (d.8) we have:

$$
a_{1}=1, a_{2} \neq 1, \frac{1}{a_{1}}+\frac{1}{a_{2}}>1 \Rightarrow \Omega_{2}(1)-\Phi_{0}(1)-\left.\frac{d}{d r} B(r)\right|_{r=1}=0,
$$

because the left-hand side is bounded by 1 for $r=1$. Hence, if (d.7) applies then: for $r \sim 1$,

$$
\begin{gathered}
\mathrm{E}\left\{r^{\mathbf{x}_{1}}\left(\mathbf{x}_{2}>\mathbf{x}_{1}\right)\right\}=\left.\frac{d}{d r} \Phi_{0}(r)\right|_{r=1}+\left.\frac{1}{2}(r-1) \frac{d^{2}}{d r^{2}} \Phi_{0}(r)\right|_{r=1}+\left.\frac{d}{d r} B(r)\right|_{r=1} \\
+\left.\frac{1}{2} r \frac{d^{2}}{d r^{2}} B(r)\right|_{r=1}+\left.\frac{1}{6} r(r-1) \frac{d^{3}}{d r^{3}} B(r)\right|_{r=1}+O\left((1-r)^{2}\right) .
\end{gathered}
$$




\section{Appendix E}

In section 7 we have defined $\lambda_{1}$ and $\lambda_{2}$. For the determination of $\tilde{M}$, cf. (8.6), we need some more detailed information. It is obtained in this appendix; further, some relations between $\lambda_{1}$ and $a_{1}, a_{2}$ are deduced. These relations are helpful in the numerical evaluation of queueing characteristics.

Denote

$$
A:=\frac{b}{2 a_{1} a_{2}}=\frac{1}{2}\left(\frac{1}{a_{1}}+\frac{1}{a_{2}}+1\right)
$$

Since for $i=1,2$,

we have

$$
a_{i} A^{2}>1
$$

$$
\widehat{\delta}_{i}=a_{i} A^{2}>1
$$

Hence, from (3.16), (7.7) and (7.8): for $i=1,2$,

$$
0<\lambda_{i}=\sqrt{\widehat{\delta}_{i}}-\sqrt{\widehat{\delta}_{i}-1}<1 \text {. }
$$

It follows that

$$
\lambda_{i}-2 \widehat{\delta}_{i} \lambda_{i}^{1 / 2}+1=0
$$

Consider the hyperbola $y^{2}-2 y x+1=0$. It has its center at the point $(0,0)$ and asymptotes $y=0, y=2 x$, the point $(1,1)$ lies on it. It is readily seen that for $i=1,2$,

$$
\widehat{\delta}_{i}>0 \Rightarrow 0<\lambda_{i}<1
$$

and $\lambda_{i}$ decreases monotonically from 1 to 0 for $\widehat{\delta}: 1 \rightarrow \infty$.

From (e.5), it is seen that a positive integer $n$ may exist such that

$$
\lambda_{1}^{n}+\lambda_{2}^{n}>1
$$

However, for $n$ sufficiently large, this inequality cannot hold for any $\widehat{\delta}_{i}>1, i=1,2$.

To derive the relation between $a_{i}$ and $\hat{\delta}_{i}$, note that from (e.1) and (e.3) we have

with

$$
a_{1}^{2}\left(\frac{\widehat{\delta}_{2}}{\widehat{\delta}_{1}+\widehat{\delta}_{2}}\right)^{2}+2(1-2 \epsilon) a_{1} \frac{\widehat{\delta}_{2}}{\widehat{\delta}_{1}+\widehat{\delta}_{2}}+1=0
$$

$$
\epsilon:=\frac{\widehat{\delta}_{1} \widehat{\delta}_{2}}{\widehat{\delta}_{1}+\widehat{\delta}_{2}}-1>0
$$

From (e.6) and (e.7), it follows readily that: for $i=1,2$,

so that

$$
a_{i}^{ \pm}=\frac{\widehat{\delta}_{i}}{1+\epsilon}\{1+2 \epsilon \pm 2 \sqrt{\epsilon(1+\epsilon)}\},
$$

It follows that

$$
\frac{1}{a_{1}^{ \pm}}+\frac{1}{a_{2}^{ \pm}}=[1+2 \epsilon \pm 2 \sqrt{\epsilon(\epsilon+1)}]^{-1} \text {. }
$$

$$
\frac{1}{a_{1}^{+}}+\frac{1}{a_{2}^{+}}<1, \frac{1}{a_{1}^{-}}+\frac{1}{a_{2}^{-}}>1 \text {. }
$$


Hence, if, cf. (2.6) and Remark 2.1,

$$
\frac{1}{a_{1}}+\frac{1}{a_{2}}>1
$$

then the relation between $a_{i}$ and $\widehat{\delta}_{i}$ is given by

$$
\begin{aligned}
a_{i}= & \frac{\widehat{\delta}_{i}}{1+\epsilon}\{1+2 \epsilon-2 \sqrt{\epsilon(\epsilon+1)}\}, i=1,2, \\
& \frac{1}{a_{1}}+\frac{1}{a_{2}}=1+2 \epsilon+2 \sqrt{\epsilon(\epsilon+1)} .
\end{aligned}
$$

\section{References}

[1] Adan, I.J.B.F., Wessels, J., and Zijm, W.H.M., Analysis of the symmetric shortest queueing problem, Stochastic Models 6 (1990), 691-713.

[2] Adan, I.J.B.F., Wessels, J., and Zijm, W.H.M., Analysis of the asymmetric shortest queue problem, Queueing Systems, Theory and Applications 8 (1991), $1-58$.

[3] Adan, I.J.B.F., A Compensation Approach for Queueing Problems, CWI Tracts \#104, Math Center, Amsterdam 1994.

[4] Blanc, J.P.C., A note on waiting times in systems with queues in parallel, J.A.P. 24 (1987), 540-546.

[5] Blanc, J.P.C., The power-series algorithm applied to the shortest-queueing problem, Op. Res. 40 (1992), 157-167.

[6] Cohen, J.W., The Single Serve Queue, Rev. Edition, North-Holland Publ. Co., Amsterdam 1982.

[7] Cohen, J.W. and Boxma, O.J., Boundary Value Problems in Queueing Systems Analysis, North-Holland Publ. Co., Amsterdam 1983.

[8] Cohen, J.W., Analysis of Random Walks, IOS Press, Amsterdam 1992.

[9] Cohen, J.W., On the determination of the stationary distribution of a symmetric clocked buffered switch, In: Teletraffic Contributions for the Information Age, Proc. 15th ITC Congress (ed. by V. Ramaswami and P.E. Wirth), Elsevier, Amsterdam (1997), 297-308.

[10] Cohen, J.W., Analysis of a two-dimensional algebraic nearest-neighbor random walk, Report BS-R9437 (Dec. 1994), Dept. of Op. Res., CWI Amsterdam.

[11] Cohen, J.W., On a class of two-dimensional nearest-neighbor random walks, Studies in Applied Probability (ed. by J. Gani and J. Galambos), Applied Prob. Trust, Sheffield (1994), 207-238.

[12] Cohen, J.W., On the analysis of the symmetric shortest queue, Quantitative Methods in Parallel Systems (ed. by F. Baccelli, A. Jean-Marie, and I. Mitrani), Springer, Berlin (1995), 141-152.

[13] Cohen, J.W., On the asymmetric clocked buffered switch, CWI Report BSR9612 (Oct. 1996).

[14] Flatto, L. and Hahn, S., Two parallel queues created by arrivals with two demands, SIAM J. Appl. Math. 44 (1984), 1041-1054.

[15] Saks, S. and Zygmund, A., Analytic Functions, Matematycznego, Warsaw 1952. 


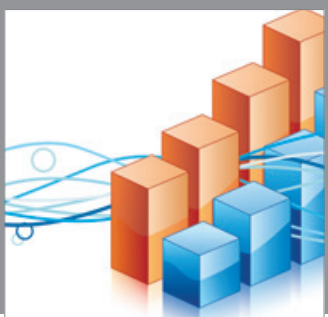

Advances in

Operations Research

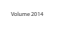



\section{The Scientific} World Journal


International Journal of

Mathematics and

Mathematical

Sciences
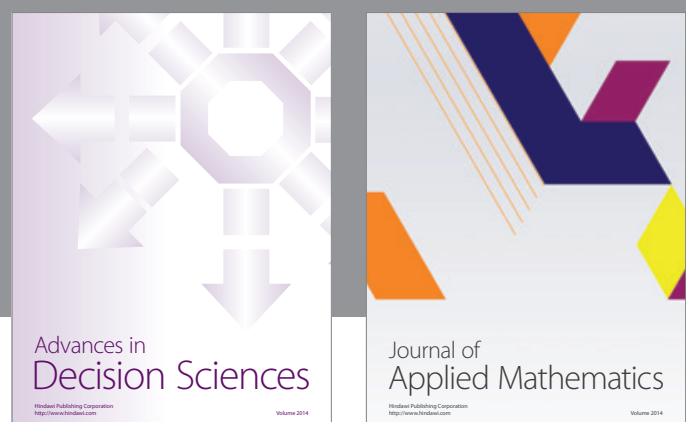

Journal of

Applied Mathematics
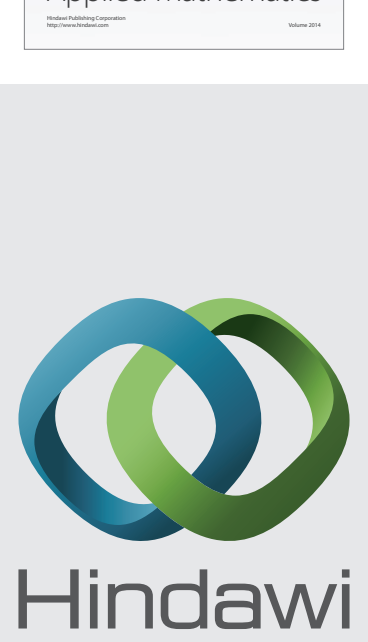

Submit your manuscripts at http://www.hindawi.com
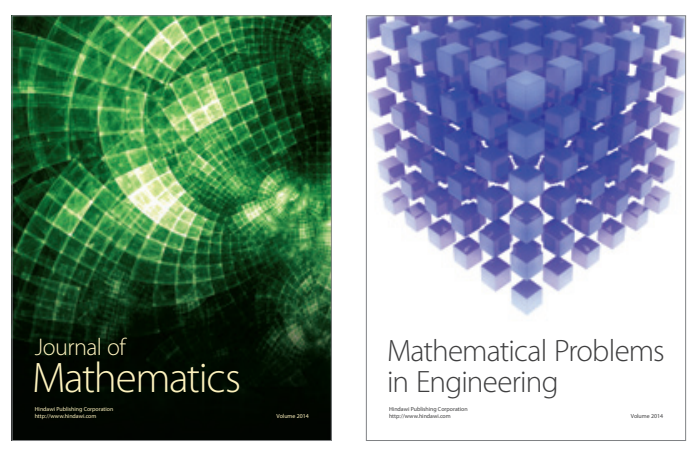

Mathematical Problems in Engineering
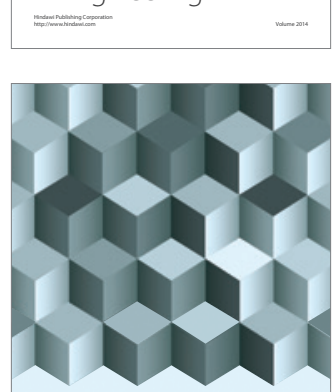

Journal of

Function Spaces
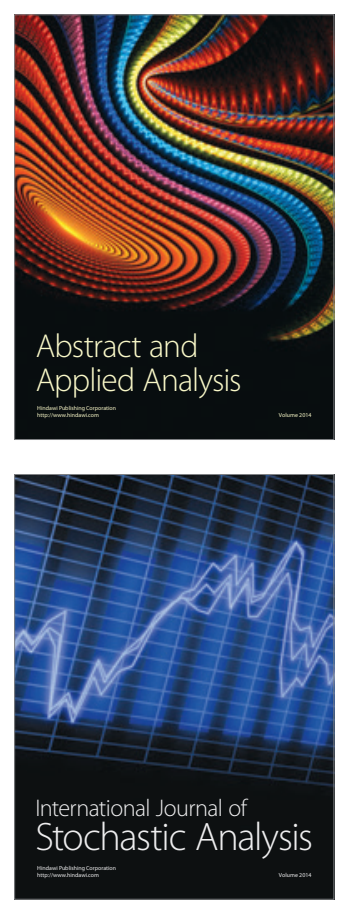

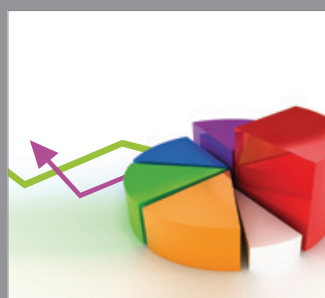

ournal of

Probability and Statistics

Promensencen
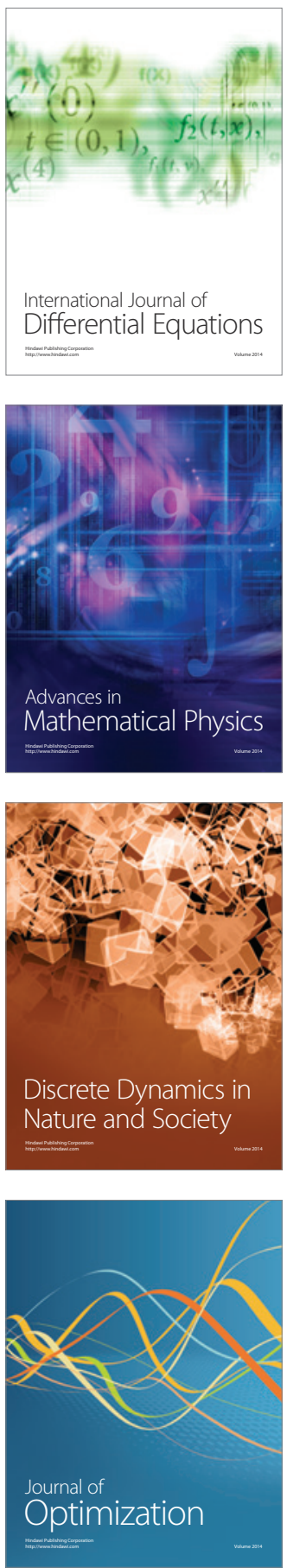